\title{
Correlation based methods for ultrasound blood velocity estimation : a comparative study
}

Citation for published version (APA):

Štúriková, E. (1997). Correlation based methods for ultrasound blood velocity estimation : a comparative study. [Doctoral Thesis, Maastricht University]. Universiteit Maastricht. https://doi.org/10.26481/dis.19970220es

Document status and date:

Published: 01/01/1997

DOI:

10.26481/dis.19970220es

Document Version:

Publisher's PDF, also known as Version of record

\section{Please check the document version of this publication:}

- A submitted manuscript is the version of the article upon submission and before peer-review. There can be important differences between the submitted version and the official published version of record.

People interested in the research are advised to contact the author for the final version of the publication, or visit the DOI to the publisher's website.

- The final author version and the galley proof are versions of the publication after peer review.

- The final published version features the final layout of the paper including the volume, issue and page numbers.

Link to publication

\footnotetext{
General rights rights.

- You may freely distribute the URL identifying the publication in the public portal. please follow below link for the End User Agreement:

www.umlib.nl/taverne-license

Take down policy

If you believe that this document breaches copyright please contact us at:

repository@maastrichtuniversity.nl

providing details and we will investigate your claim.
}

Copyright and moral rights for the publications made accessible in the public portal are retained by the authors and/or other copyright owners and it is a condition of accessing publications that users recognise and abide by the legal requirements associated with these

- Users may download and print one copy of any publication from the public portal for the purpose of private study or research.

- You may not further distribute the material or use it for any profit-making activity or commercial gain

If the publication is distributed under the terms of Article $25 \mathrm{fa}$ of the Dutch Copyright Act, indicated by the "Taverne" license above, 
Correlation Based Methods for Ultrasound Blood Velocity Estimation

A Comparative Study 
ISBN 90-9010267-1 


\title{
Correlation Based Methods for Ultrasound Blood Velocity Estimation
}

A Comparative Study

\section{Proefschrift}

\author{
ter verkrijging van \\ de graad van doctor aan de Universiteit Maastricht, \\ op gezag van de Rector Magnificus, Prof.mr. M.J. Cohen, \\ volgens het besluit van het College van Decanen, \\ in het openbaar te verdedigen \\ op donderdag 20 februari 1997 om 14.00 uur
}

door

Elena Štúriková 


\section{Promotores:}

Prof.dr.ir.drs. O.J. Vrieze

Prof.dr.ir. A.P.G. Hoeks

Co-promotor:

Dr.ir. R. Luesink

Beoordelingscommissie:

Prof.dr.ir. A. Hasman (voorziter)

Prof.dr.ir. M.G.J. Arts

Dr.ir. P. Boekhoudt

Prof.dr.ir. O.H. Bosgra (Technische Universiteit Delft)

Financial support by the Netherlands Heart Foundation for the publication of this thesis is gratefully acknowledged. 


\section{Acknowledgments}

This thesis is the culmination of the academic experience I have acquired during my stay at the Department of Mathematics of the Faculty of General Science at Maastricht University since September 1992. The work for this thesis was conducted in cooperation with the Department of Biophysics of the Faculty of Medicine of the same university. Inspiration, encouragement and support have come from many people and several institutions. They have certainly all contributed to the development of this thesis. It is a pleasure for me now to express my gratitude to them.

My deepest thanks go to Prof.dr.ir.drs. O.J. Vrieze, Prof.dr.ir. A.P.G. Hoeks, and Dr.ir. R. Luesink. I thank Rob Luesink for the constant guidance he gave me during the work for this thesis. His helpful suggestions and inspiring ideas were decisive for the progress of my research. It was Arnold Hoeks who provided the topic for my Ph.D. project and who introduced me to the fascinating world of ultrasound and ultrasonic measurements of blood flow. I thank him for this, for the many interesting discussions and for his assistance during the writing of this thesis. I thank Koos Vrieze for his contribution to stimulating discussions on the topic covered in this thesis. His personal enthusiasm and positive attitude towards this research supported my strong commitment to achieving the Ph.D. degree.

I wish to thank all the members of the Department of Mathematics for creating a pleasant working atmosphere. I thank my office-mate Ralf Peeters for his willingness to answer all my questions and for his assistance with programming issues in Matlab and $I^{\prime} I_{\mathrm{E} X} X$. I enjoyed and benefited a lot from sharing the office with him. I am also grateful to Piet Boekhoudt for his continuous interest in the development of my research and for his support with Matlab issues. My thanks are also due to Jeroen Rutten whose expertise on ${ }_{A} \mathrm{~T}_{\mathrm{E}} \mathrm{X}$ helped me out on several occasions. I am grateful to Jean Derks for his help during hardware emergencies with my computer. I thank Marijke Verheij and Ruth Schrijen for their kind and helpful presence and for their assistance with my administrative concerns.

I would like to express my gratitude to the members of the assessment committee, namely Prof.dr.ir. A. Hasman, Prof.dr.ir. M.G.J. Arts, Dr.ir. P. Boekhoudt and Prof.dr.ir. O.H. Bosgra for their careful reading of the manuscript and for their insightful comments. 
I am grateful to Mary Kelly for her time and effort she spent trying to improve my written English.

Reflecting on the beginning of my international professional career I feel indebted to Associate Professor Stefan Kozák, my former supervisor at the Faculty of Electrical Engineering of the Slovak Technical University, for his encouragement to pursue my career abroad. His approval of the idea of continuing my studies at the Eindhoven International Institute and then at Maastricht University was quite significant for me.

I am grateful to the former Dutch Systems and Control Theory Network and its surcessor the Dutch Institute on Systr'ms and Control, for giving me the opportunity to attend high level graduate courses and for providing the financial support to participate at the international conference in Canada.

That this Ph.D. project has been accomplished is also due to the Department of Biophysics of the Faculty of Medicine at Maastricht University and its Professor Arnold Hoeks. I thank him for arranging the financial support to enable my contract at the university to be extended for six months.

I gratefully acknowledge the financial support of Pie Medical Benelux B.V. for the publication of this thesis.

I thank all my colleagues and many other people who have tried to make my stay in the Netherlands enjoyable. I am grateful to my former colleague Marlies Haenen and her family for their indispensable assistance in helping me settle in in Maastricht.

Finally I thank my parents for giving me the opportunity to gain a university education. I thank my mother for the huge number of letters she has written to me from Slovakia keeping me informed about my family.

Maastricht, January 1997

Elena ŠtúRIKová 


\section{Contents}

Acknowledgements $\quad$ v

Contents vii

1 Introduction 1

1.1 Topic Outline . . . . . . . . . . . . . . . . . . . 1

1.2 Synthesizing Theoretical Concepts .............. . . 3

1.3 Thesis Organization . . . . . . . . . . . . . . . 4

2 Physical Principles of Ultrasound Blood Flow Measurement $\quad 7$

2.1 Introduction . . . . . . . . . . . . . . 7

2.2 Basic Concepts in Ultrasound Blood Flow Measurement . . . . . . . 8

2.2.1 Sound Propagation ... . . . . . . . . . . . . . 8

2.2.2 Ultrasound and its Interactions with Tissue . . . . . . . . . 9

2.2 .3 Scattering from Blood . . . . . . . . . . . . . 10

2.2 .4 The Echo Principle . . . . . . . . . . . . . . . . . 11

2.3 Generation and Detection of Ultrasound . . . . . . . . . 11

2.3 .1 Ultrasonic Transducer . . . . . . . . . . . . . . . . 13

2.3 .2 Sound Beam and Resolution . . . . . . . . . . . . . 13

2.3 .3 Signal Visualization . . . . . . . . . . . . . . . . . . 14

2.4 Physical Properties of the Pulsed RF Signal . . . . . . . . . . . . 15

2.4.1 Spatial and Temporal Properties of the RF Signals . . . . . 15

2.4.2 Temporal Properties of the RF Signals . . . . . . . . . . 18

2.4.3 Spatial Properties of the RF Signals . . . . . . . . . . . . 20

2.4.4 Pulse Repetition Frequency and Aliasing . . . . . . . . . 21

2.4 .5 Noise Sources and Signal-to-Noise Ratio . . . . . . . . . 22

2.5 Summary . . . . . . . . . . . . . . . . . . 23

3 Principles of Signal Processing for Blood Velocity Estimation 25

3.1 Introduction . . . . . . . . . . . . . . . 25

3.2 Form of the Data . . . . . . . . . . . . . . . 26

3.3 Related Statistical Principles . . . . . . . . . . . . . . . . . . 27

3.3.1 Correlation . . . . . . . . . . . . . . . . . 28

3.3.2 Quality Measures in Data Evaluation . . . . . . . . . . . . 29

3.3.3 Cramér-Rao Inequality and Efficiency. . . . . . . . . 30 
3.4 Complex Envelope Transformation ... . . . . . . . . . . . . 31

3.5 Main Processing Concepts for Blood Velocity Estimation . . . . . . . 33

3.5.1 Processing of Low Frequency Signal . . . . . . . . . . . . . 33

3.5 .2 Processing of High Frequency Signal . . . . . . . . . . 35

4 A Simulation Model of the Backscattered Signal from Blood $\quad 37$

4.1 Introduction . . . . . . . . . . . . . . . . . 37

4.2 Assumptions of the Model . . . . . . . . . . . . . . . 38

4.3 Input Parameters for the Model . . . . . . . . . . . . . . . . . 40

4.4 Modeling of the Backscattered Signal from Blood . . . . . . . . . . 42

4.4.1 Generation of the Waveforms . . . . . . . . . . . . . . 43

4.4 .2 Generation of Successive Signals . . . . . . . . . . . . 44

4.4.3 Generation of Signal with the Desired SNR . . . . . . . . . 47

4.5 Example of Simulated Signal . . . . . . . . . . . . . . . 47

5 The Maximum Likelihood Method 49

5.1 Introduction . . . . . . . . . . . . . . . . . . . . 49

5.2 Maximum Likelihood Blood Velocity Estimator . . . . . . . . . . 51

5.2 .1 Theoretical Derivation . . . . . . . . . . . . . . 51

5.2.2 Formulation of Likelihood Function for Blood Velocity Fstimation . . . . . . . . . . . . . . . 53

5.2.3 Implementation of Maximum Likelihood Estimator . . . . . . 55

5.3 Statistical Properties of the Maximum Likelihood Estimator . . . . . 59

5.4 Performance of the Maximum Likelihood Estimator . . . . . . . . . . 63

5.4 .1 Effect of Velocity . . . . . . . . . . . . . 63

5.4.2 Effect of the Processing Paraneters . . . . . . . . . . 67

5.4.3 Effect of the System Properties . . . . . . . . . . . . . . 69

5.4 .4 Summary ....................... 73

6 Cross-Correlation Model Method $\quad \mathbf{7 5}$

6.1 Introduction . . . . . . . . . . . . . . . 75

6.2 Model of the Cross-Correlation Function . . . . . . . . . . . 76

6.3 Cross-Correlation Model Estimator of Blood Velocity . . . . . . . . 77

6.3.1 Narrowband CCM Velocity Estimator . . . . . . . . . . . . 78

6.3.2 Wideband CCM Velocity Estimator . . . . . . . . . . . . . 79

6.4 Statistical Properties of the CCM Estimator . . . . . . . . . . . . . 81

6.5 Performance of the Wideband CCM Estimator . . . . . . . . . . . . 83

6.5 .1 Effect of Velocity . . . . . . . . . . . . . . . 83

6.5.2 Effect of the Processing Parameters . . . . . . . . . . 85

6.5.3 Effect of the System Properties ... . . . . . . . . . 89

6.5.4 Sensitivity Analysis for the Shape of the Spectrum . . . . . . 91

6.5 .5 Summary . . . . . . . . . . . . . . . . 95 
7 Comparative Evaluation of Correlation Based Velocity Estimators 97 7.1 Introduction . . . . . . . . . . . . . . . . . 97

7.2 Effect of Frequency Dependent Attenuation . . . . . . . . . . . . . . 98

7.3 Comparison of Performance with the Theoretical Limit . . . . . . . . 101

7.4 Resolution and Implementation Aspects . . . . . . . . . . . . . . . 106

7.4.1 Precision and Axial Resolution . . . . . . . . . . . . 106

7.4.2 Precision and Temporal Resolution ............ . 108

7.4 .3 Computational Effort . . . . . . . . . . . . . 109

8 Concluding Remarks

$\begin{array}{ll}\text { Bibliography } & 117\end{array}$

$\begin{array}{ll}\text { Summary } & 123\end{array}$

Samenvatting (Summary in Dutch) $\quad 125$

$\begin{array}{ll}\text { Zhrnutie (Summary in Slovak) } & 127\end{array}$

$\begin{array}{lr}\text { About the Author } & 129\end{array}$ 



\section{Chapter 1}

\section{Introduction}

In this chapter we present three outlines of the contents of this thesis. The first is a topical outline in which we develop a brief description of application of ultrasound for blood velocity measurements and formulate the goal of this study. The second is a synthesizing outline in which we point out theoretical concepts applied in approaching the problem of blood velocity estimation. The third is a chronological outline in which we explain the structure of the material presented.

\subsection{Topic Outline}

Ultrasound waves are those sound waves that the human ear cannot hear, that is, these waves oscillate at frequencies greater than $20 \mathrm{kHz}$. Their properties are those common to all sound waves. As waves, they propagate through the medium, and are reflected at an acoustic interface.

Ultrasound is a standard clinical tool in the diagnosis and treatment of illness and injury. The widely used method for making ultrasonic measurements is based on the reflections of ultrasound (echoes). An electric pulse is applied to an ultrasonic transducer which is in acoustic contact with the sample volume to be examined. The transducer converts the electric pulse into an acoustic pressure wave that is transmitted into the body. The ultrasound energy is reflected and scattered by both moving and stationary structures along the ultrasound beam. The energy that finds its way back to the transducer is related to the acoustic impedance mismatch of the reflecting structure in relation to its environment. The transducer receives and converts the reflected pressure wave into an electric signal. The objective is then to extract the desired information by analyzing the received signals, and so to obtain information about structure that is hidden from direct view.

Perhaps the most familiar use of ultrasound is in the noninvasive imaging of different parts of the body. In these techniques the reflected sound from tissue interfaces is used to generate an image. With known sound velocity, the time elapsed between transmission of the wave and reception of the echo can be converted to a distance or depth measurement. The echo may be displayed on a screen at a corresponding depth in brightness-mode ( $\mathrm{B}$-mode). The brightness of the dot corresponds to the 
strength or amplitude of the signal. When the body is scanned from different directions or positions, the two-dimensional image of a planar section of an investigated anatomical structure can be obtained.

Ultrasound does, however, have other applications in medical diagnosis, such as in tissue characterization and measurement of tissue motion. The measurement of tissue motion may include the motion of physically active organs such as the heart, cardiac valves and arterial walls. With ultrasound, the assessment of hemodynamic variables is also possible, for example of blood flow velocity. Blood velocity is an important parameter in diagnosing vascular diseases since changes in blood flow caused by pathology (atherosclerosis for example) can be directly observed through changes in the speed of blood.

With respect to blood flow velocity measurement, two kinds of modes can be distinguished: the continuous wave (CW) mode and the pulsed wave (PW) mode. In CW mode ultrasound is transmitted and received continuously by means of two transducers. The continuous signal is obtained from the sample volume where the transmitted beam overlap the sensitivity region of the receiving transducer. With a continuous wave system we observe all blood motion along the beam, i.e. it is not possible to know the range from which the measurement was obtained.

In PW mode short bursts of sound are emitted with pulse repetition frequency. Between transmissions, the transducer is switched to reception mode to catch the signals backscattered from blood. The position of insonified regions can be localized since as the depth of the region of interest increases, the time before receiving the echo lengthens. PW data consists of a sequence of radio frequency (RF) signals resulting from repeated insonification of a sample volume. Since, contrary to $\mathrm{CW}$, the PW system is capable of localizing the origin of echoes along the sound beam axis, it is widely used at the present time. For the same reason PW mode is also employed in technology known as color flow imaging. Ultrasound blood velocity measurement is coded in color and added in real time to B-mode gray-level anatomical images. This ultrasound system provides the clinical community with a tool for rapid and inexpensive investigation of the human circulatory system.

Of particular interest to us in this thesis are methods for velocity estimation applied to pulsed ultrasound signals i.e. signals obtained in PW mode. A number of techniques can be employed to extract velocity information from such signals. The most salient are summarized in Section 3.5. Many of the existing techniques involve performing correlation. However, the diversity of descriptions and assumptions of the results reported in the literature do not allow a straight comparison of their performance. It is not possible to deduce which method is superior. The reason for this is that comparison of different techniques is hampered by the lack of unified methodology for performance investigation and for results evaluation. Therefore, a comparison of various techniques in identical situations and in identical way would be desirable.

It can be recognized that the drawbacks of the existing processing methods include limitations arising from the conflicting requirements for velocity precision, and 
axial (along the axis of the ultrasound beam) and temporal resolutions. In practice, there is a direct trade-off between resolution and velocity precision. An increase in the transmitted signal bandwidth which would result in improvement of the axial resolution may decrease velocity precision. Furthermore, an increasing size of the observation window (in depth and time) improves precision and at the same time decreases resolution (spatial, temporal). Good resolutions are important for visualizing velocity measurements in color flow imaging.

The ideal velocity estimation technique will provide an acceptable precision for wideband signals, for short observation windows (in depth and time), under low signal-to-noise ratios, and for a wide range of mean velocities and degrees of velocity dispersion. Another criterion, of course, is the feasibility of real-time implementation of the algorithm for velocity estimation to be applied in color flow imaging.

Our goal is to investigate performance of existing methods for blood velocity estimation, to address the robustness properties of the methods to their assumptions, and to compare various techniques in identical terms for the effect of processing and system parameters. Furthermore, the goal is to propose a new processing technique for possible improvement in accuracy and resolution in accordance with the requirements mentioned above.

\subsection{Synthesizing Theoretical Concepts}

This study is concerned with methods for processing of ultrasound signals in order to assess blood velocity. The subject involves several concepts, as we shall point out in this section.

The idea of the study is based on transmitting a beam of ultrasound into the body and collecting and analyzing the returning echoes. Thus the relevant concept within this subject is a concept of signals and systems. These notions are extremely general concepts that arise in a wide variety of fields, for example in communication, acoustics, chemical process control, speech processing. Although the physical nature of the signals and systems that arise in the various disciplines may be different, they all have basic features in common. The systems respond to particular signals by producing other signals. Therefore, signals typically contain information about the behavior or nature of the system. A human circulatory system is itself an example of a system, which in this case responds to applied ultrasound signals. Echoes as a function of depth and time are examples of signals.

In the many contexts in which signals and systems arise, there are a variety of problems and questions that we may consider. In our case, the signals (echoes) are the carriers of the useful information about the human circulatory system and the essential problem is to extract this information of interest (the velocity of blood). However, the required information cannot be read directly from the signals but can be obtained by suitable processing of the observed signals. A familiar notion associated with this problem is signal processing. The design problem in signal processing is 
to find a signal processor (filter, receiver) whose input is a signal and whose output is the desired information that is to be extracted from the signal. A design should reflect the properties of the signal. To describe these properties, a model of the signal is needed.

In this study we use models (model of the backscattered signal, or model of the cross-correlation function) whose variables (time, depth) and parameters (velocity) have a physical meaning. Then we apply statistically based methods (the maximum likelihood estimation method, or correlation function estimation) to estimate an unknown parameter: the velocity. The next stage involves validation of the model where the criterion of acceptance is given by the precision of the velocity estimator. The model obtained is tested to see whether it is an appropriate representation of the system. If this is not the case, some other model (or structure) must be considered, its parameters estimated, the new model validated, etc. The procedure consisting of a model choice, criterion, parameter estimation and a model validation has a certain affinity with the system identification procedure.

As we have seen, an application of the system identification methodology leads to the design of the possible signal processor. The output of the signal processor is a velocity estimate, that provides such necessary information about the blood velocity to enable us reproduce the local blood flow.

The purpose of the above discussion was to point out that the subject of this thesis belongs to a class of applications in which the concepts and techniques of signal analysis and system identification arise. The set of ideas embodied in these techniques and the specific techniques themselves proves to be of value in approaching and analyzing the problem of blood velocity estimation. It demonstrates the practicability of many of the theoretical concepts.

\subsection{Thesis Organization}

The thesis is organized in the following manner.

Chapter 2 gives survey of some elements needed from ultrasound physics in order to understand the backscattering process from blood. It discusses some basic physics encountered in the application of ultrasound for blood flow measurement. (Section 2.2) like the propagation phenomenon, the echo principle, the interaction of ultrasonic wave and blood. Furthermore, it gives an overall view of the technical principles in generation and detection of ultrasound (Section 2.3). The central issue is an ultrasonic transducer that converts electric signal to ultrasonic and vice versa. This chapter also includes an extensive study of the features of backscattered signals received in pulsed wave measurement mode (also called radio frequency ( $R F$ ) signals). In pulsed wave systems ultrasound pulses are transmitted with a pulse repetition frequency. After each pulse has been emitted, the echo signal is received, yielding a set of RF signals that is available for further processing. A return due to the $k$-th transmitted pulse is a function of depth. The subsequent return is separated 
by the pulse repetition period and exhibits gradual translation with respect to the previous pulse return. This translation is due to the changing distance between the transducc and groups of moving scatterers and carries information about the velocity of moving scatterers in the area under investigation. The underlying properties with respect to both variables: depth and time, and their frequency counterparts: spatial and temporal frequency, are described in Section 2.4.

Chapter 3 assembles some principles, and reference-points for following chapters. First, it describes the principles and stages of signal processing for blood velocity estimation. It introduces several matters associated with RF signal processing, such as form of data and sampling requirements (Section 3.2), and useful signal transformation for pre-processing of the data (Section 3.4). Second, this chapter introduces some of the statistical principles in Section 3.3 (like correlation, types of error in data evaluation and a concept of estimation efficiency). These statistical results will be applied in the performance analysis and comparison of the reported methods in latter chapters. Finally, Section 3.5 contains a summary of existing methods for blood velocity estimation given within a non-traditional classification scheme based on the character of input data. Existing methods are reviewed for two cases of processing: low and high frequency signals.

Chapter 4 concerns a simulation model of the backscattered signal that will provide data for the analysis of various methods in subsequent chapters. The simulation procedure (described in Section 4.4) is based on the fact that the RF signal is formed by incoherent scattering of ultrasound from a large number of randomly located blood cells. Input parameters for the simulation model are specified in Section 4.3. The modelling procedure was introduced in Hoeks et al. [29] and has already been applied in various studies. Here, it is presented in a slightly different fashion and studied in sufficient detail, based on the properties of the RF signal outlined in Section 4.2. Two-dimensional spectral characteristics of the $\mathrm{RF}$ signal with respect to spatial and temporal frequencies are examined in Section 4.5. The frequency content of the signal generated by the simulation procedure is concentrated around a straight line in the frequency plane. This line passes through the origin and has a slope linearly proportional to the scatterers' velocity as was theoretically established by Wilson [71], and Loupas et al. [44].

Chapters 5 and 6 are both concerned with estimation methods. Chapter 5 deals solely with the method based on the maximum likelihood criterion. The theoretical derivation of the likelihood function (Section 5.2.2) is based on the slowly fluctuating point target approximation commonly applied in radar. This approximation considers a group of scatterers within a sample volume as a point target, slowly moving at a constant velocity. The reflective characteristics of the target are modeled as a (time independent) random variable. In practical situation, however, the point target assumption is violated, and the scattering characteristics of blood are to be regarded as (time dependent) stochastic process. It interests us how this incompatibility influences the performance of the ML method. The scheme for maximum likelihood estimation of blood velocity consists of a number of parallel processing lines each tuned to a different velocity. In such a way a discrete approximation of the likeli- 
hood function is obtained. The estimate of velocity is chosen as that value of velocity which gives the maximum of the (discrete) likelihood function. This technique has already been proposed for blood velocity estimation by Ferrara and Algazi in [19] and [20]. Here, it is re-examined and applied to our simulated signals. Emphasis is put on the investigation of the robustness of the method to the slowly fluctuating point target assumption. The performance of the method is evaluated in Section 5.4 for the effects of various system and processing parameters.

Chapter 6 presents a new algorithm based on the cross-correlation model (CCM) approach. The model of the cross-correlation function is presented in Section 6.2. In a previous study [29] the velocity estimator has been derived under the narrowband assumption for the transmitted signal. Here, for the derivation of the new estimator, the narrowband assumption is dropped and a velocity estimator is derived in Section 6.3.2. The CCM approach does not demand the calculation of the complete correlation function. Correlations at six lags are sufficient to solve for the velocity. The resulting estimator employs a restricted number of simple operations, so that real-time implementation is feasible. The robustness of the wideband CCM method in relation to the assumption on the symmetric shape of the spectrum is indicated in Section 6.5.4. The results of an analysis of the effects of various processing and system parameters are reported in Section 6.5 .

Chapter 7 concentrates on a comparison of both preceding methods. The effect of frequency-dependent attenuation is investigated in Section 7.2. A particular attention is given to the comparison of performance with the theoretical limit on standard deviation described by the Cramér-Rao lower bound in Section 7.3. First, the lower bound is derived, then the results obtained for both methods are compared with this theoretical limit. Recalling the results obtained in Chapters 5 and 6 , the resolution aspects in relation to the velocity precision are addressed in Section 7.4. From the comparison of the reported methods it can be concluded that the wideband CCM estimator provides better precision than the ML estimator under all conditions considered.

Chapter 8 concludes the thesis with an evaluation of the output of this study. 


\author{
Chapter 2
}

\title{
Physical Principles of Ultrasound Blood Flow Measurement
}

\subsection{Introduction}

Nowadays, in many cases the use of ultrasound diagnostic equipment is considered necessary for optimal patient treatment. Ultrasound is particularly suited to situations where exposure to ionizing radiation or injection of contrast material is undesirable. In general, there are four diagnostic parameters [7] that can be obtained by ultrasound: structure tomography that yields the cross-sectional anatomy of an organ; structure motion that allows observation of moving objects such as the cardiac valves; blood velocity that helps to observe the changes in blood flow; and tissue characterization that provides information on properties of a iissue. Although the principles used in medical ultrasound have been recognized for a long time, the practical development of instrumentation and application in cardiology have taken several decades. A good review of the history and development can be found in [70]. That the diagnostic ultrasound systems can operate in real-time makes it possible to visualize the dynamic structures. Therefore, ultrasound may be used for the study of various types of motion within the body. It is applied for instance in cardiology for the detection and quantification of flow in the heart, arteries and veins. The study of blood flow at a given site can provide valuable information on the vascular and cardiac pathologies. Detection and quantification of flow in the heart, arteries and veins cannot be done directly by ultrasound but must be derived from velocity measurements. The objective is to obtain measurement of flow velocity and interpret them in terms of physiologically significant variables.

Regardless of the particular medical application of ultrasound, all applications require the transmission and capture of ultrasonic signals. After capture, the signal must be processed in some way to extract the desired information. The correct interpretation of the results obtained from ultrasound equipment depends on understanding both the physical mechanism and signal processing methods. The aim of this chapter is to review (and refresh) some physical knowledge of ultrasound and to 
describe associated matters, specifically from the point of view of blood flow measurements. The first section (2.2) includes the physical concepts used in applications of ultrasound for blood flow measurements, and the next one (Section 2.3) describes the technical aspects used in generation and detection of ultrasound. The physical features of the backscattered signal that results from the interaction of ultrasound wave and blood are covered in Section 2.4.

\subsection{Basic Concepts in Ultrasound Blood Flow Measure- ment}

This section briefly discusses some basic physics encountered in the use of ultrasonic techniques with respect to our primary interest, blood flow measurement. For an in-depth study of the physics of ultrasound we refer to [69].

We will cover the wave propagation phenomenon (Section 2.2.1), as well as properties of sound waves. The ultrasonic phenomena occuring in tissue, such as attenuation, absorption, reflection, and scattering will be described in Section 2.2.2. The interaction of ultrasonic wave and blood will be analyzed in Section 2.2.3. Furthermore, in Section 2.2.4 we will describe how the measured echo travel time is converted to distance from the transducer.

\subsubsection{Sound Propagation}

Sound waves represent a pressure disturbance that is propagated through a medium (such as air, water, human tissue). These pressure waves result from a force acting on the molecules of the medium, causing them to oscillate about their mean position. A wave is propagated due to the interaction of neighbouring molecules. There is no flow of molecules; rather, the effect is transmitted over long distances because of neighbour-to-neighbour interactions. Waves are divided into two basic types: longitudinal and transverse. Longitudinal waves are those for which the oscillatory motion of the particles of the medium is parallel to the direction of propagation of the wave energy. Transverse waves are those for which particle motion is perpendicular to the direction of propagation of the wave energy. Ultrasound waves used in medical applications are, with very few exceptions, longitudina] [56]. (Bone is the only biological tissue that can cause the production of transverse waves, which are sometimes referred to as shear waves or stress waves [33].)

When the driving force oscillates sinusoidally, the particles respond in the same way. Suppose the displacements of the particle versus both distance, $z$, and time, $t$, are sinusoidal. Sound, as the pressure wave, transports energy and can be described in terms of a number of wave parameters:

- the amplitude or peak pressure is a measure of the strength of the sound wave;

- the wavelength $\lambda$ is the distance the wave travels during a single cycle; 
- the frequency is defined as the number of cycles passing a given point in one unit of time (usually seconds). Since the period $T$ is the time it takes to complete a full (sinusoidal) cycle, the frequency is the inverse of the period $\left(T^{-1}\right)$. The unit of frequency is Hertz $(\mathrm{Hz})$, which is equal to one cycle per second.

The wave will propagate through the medium at a rate or speed called the wave velocity, c. It is virtually independent of frequency, and depends on the density and compressibility of the medium. In general, because the compressibility is low, most solids have greater velocities than do liquids or gases. Biological tissue behaves in a manner similar to liquids for the transference of sound. The sound propagation velocity in tissue is approximately $1540 \mathrm{~ms}^{-1}$. The velocity of sound remains constant for a particular medium. The velocity $c$ is equal to the frequency $f$ times the wavelength $\lambda$ stated mathematically as

$$
c=f \lambda
$$

A sound wave is a pressure disturbance propagating through the medium since neighbouring particles mutually interact with each other. The oscillation of the material sets up periodic variation in pressure within the medium. The pressure change, $p$, associated with this disturbance is related to the change in particle velocity, $v$, by the following equation:

$$
p=Z v
$$

where the quantity $Z$ is characteristic (aroustic) impedance. It is a measure of the resistance to sound passing through the medium and is expressed as the product of a,coustic velocity, $c$, and the density of the medium, $\rho_{m}$

$$
Z=\rho_{m} c
$$

High-density materials have high velocities and therefore high acoustic impedances. Similarly, low-density materials such as gases have low acoustic impedances. The differences in acoustic impedance between two media determine the amount of the reflection which occurs at their boundary (e.g. acoustic interface).

\subsubsection{Ultrasound and its Interactions with Tissue}

The ultrasound pulses which are transmitted and received for diagnostic purposes differ only in frequency from audible sound. By definition, all sound having a frequency above $20 \mathrm{kHz}$ is called ultrasound. For medical purposes, frequencies between $2 \mathrm{MHz}$ and $10 \mathrm{MHz}$ are used [17]. These frequencies correspond to the wavelengths between $0.77 \mathrm{~mm}$ and $0.154 \mathrm{~mm}$ (provided the ultrasound velocity is $1540 \mathrm{~ms}^{-1}$ ). When ultrasound waves propagate through a medium the progressive loss of amplitude is called attenuation. The sound energy contained in the transmitted pulse is reduced as a function of distance. The energy may be diverted by reflection or scattering or absorbed by the medium and converted to heat. We will now describe all these processes that cause attenuation. 
Absorption is a process in which energy is deposited in a medium by transforming ultrasonic energy into other energy forms, primarily heat. It is strongly dependent on the frequency. Higher frequencies are absorbed more rapidly, meaning that the reflected sound waves from deep-lying interfaces would be too weak to be detected. As a result, the penetration depth at high frequency is limited.

Reflection is the primary interaction used for many types of diagnostic applications. It is an interaction that results in part of the sound being redirected into the media from which it came (as an echo) after striking an acoustic interface - a boundary between two media. Assuming normal incidence (transmitter and receiver at the same position) the reflectivity at boundaries depends on the difference in acoustic impedance of these media. Two types of reflections can be observed: specular and nonspecular. Specular reflections are created when the interface is large compared to the wavelength. Another phenomenon, scattering, occurs when an ultrasonic wave travelling through a medium strikes a discontinuity of dimensions similar to or less than a wavelength in size. Some of the energy of the wave is scattered in all directions. This wave behavior is also called nonspecular reflection. The discontinuities may be changes in density or in compressibility in a medium or both. They are usually considered to be a random distribution of closely packed scattering centres. The red cells in blood, singly or in groups, act as scattering centres which produce the signals used for blood velocity measurements, as we shall see in next section. In contrast to specular echoes, the amplitude of non-specular echoes is much lower than the specular one since the beam energy is spread out in many directions. Moreorcr, the difference in acoustic impedance may be small.

In diagnostic ultrasound a combination of specular reflections from boundaries and backscatter echoes originating within the tissue is received.

\subsubsection{Scattering from Blood}

In ultrasound blood flow measurements, backscattered signals originating from blood are used to extract the velocity information. This signal is formed by the scattering of an ultrasound beam from a group of red blood cells.

Despite of its appearance, blood is not a homogeneous liquid, but a suspension of cells and other particles in a surrounding liquid, the plasma. The so-called constituting elements of blood are the erythrocytes (red blood cells), the leukocytes (white blood cells), and platelets. Scattering by blood is well understood, and is caused by the differences in density and compressibility between the red cells and plasma [1]. It is because the erythrocytes are much more numerous than the slightly larger leukocytes, and significantly larger than the platelets. The behavior of the erythrocytes as targets depends on their size, concentration and acoustic properties, and the acoustic properties of the plasma.

Undeformed erythrocytes are flexible biconcave discs and even at the highest ultrasound frequencies used for blood velocity measurements their diameter is much smaller than an ultrasound wavelength [17]. The scattering of waves by particles 
that are small in comparison to the wavelength is usually referred to as Rayleigh scattering. Two of its important characteristics are that the shape of the scatterers is unimportant, and that the scattered power is proportional to the fourth power of frequency [17].

There are likely to be numerous scatterers $\left(>10^{6}\right)$ within an ultrasonic sample volume. Using the superposition principle, the total backscattered signal power is proportional to the total volume of scatterers observed. The scattered signal is thus composed of the sum of the signals returned by a large number of scatterers present in the sample volume.

\subsubsection{The Echo Principle}

In ultrasonic echography the echo travel time is used to build up a correct echo image. First, an ultrasonic signal is transmitted into the body. It strikes an interface in the body (acoustic mismatch between two media), and some of the beam is reflected back as an echo. This signal results in ultrasonic detection of interfaces within the body. Assuming that the transmitter and receiver are at the same position it is possible to determine the distance to an interface (i.e. the depth of the interface) using the technique called echo ranging: with known sound velocity, $c$, the time elapsed between transmission of the pulse and reception of the echo, $t$, can be converted to a. distance, $d$, traveled by the sound beam according to the formula

$$
d=\frac{1}{2} c t
$$

A factor $1 / 2$ is due to the round trip travel time. The sound wave passes the distance twice (omission and reception).

In general, the principle of all echo systems is based on the time delay measurement between a transmitted pulse and the detected echo.

\subsection{Generation and Detection of Ultrasound}

Generation and detection of ultrasound is possible due to the piezoelectric effect. It occurs in certain natural materials when an electric field is applied in a certain direction. The electric field causes a change in physical dimensions, an expansion or contraction. The same type of material will generate an electric field if it is compressed or expanded. This means that the same material may be used for both transmission and reception of ultrasound.

The piezoelectric clement is the central part of an ultrasonic transducer which is described in Section 2.3.1. This device generates an ultrasonic beam. The primary objectives are to produce a unidirectional ultrasonic beam and a beam of uniform intensity. What frequencies are required to produce the resolution necessary for diagnostic ultrasound is discussed in Section 2.3.2. In Section 2.3.3 it is explained how the returned echo can be displayed (visualized). 


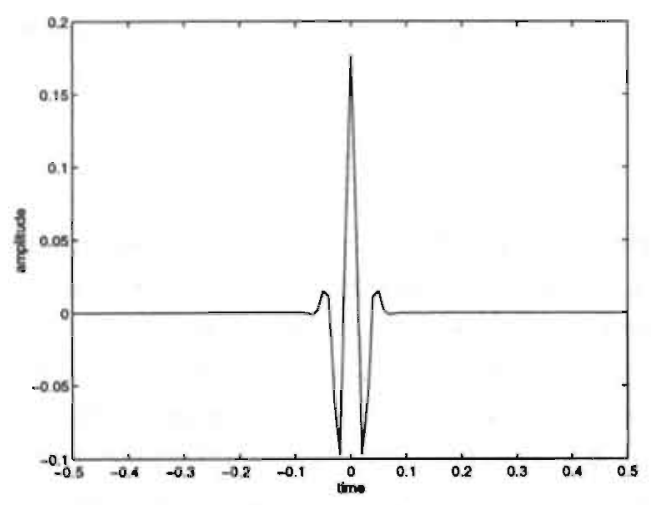

(a)

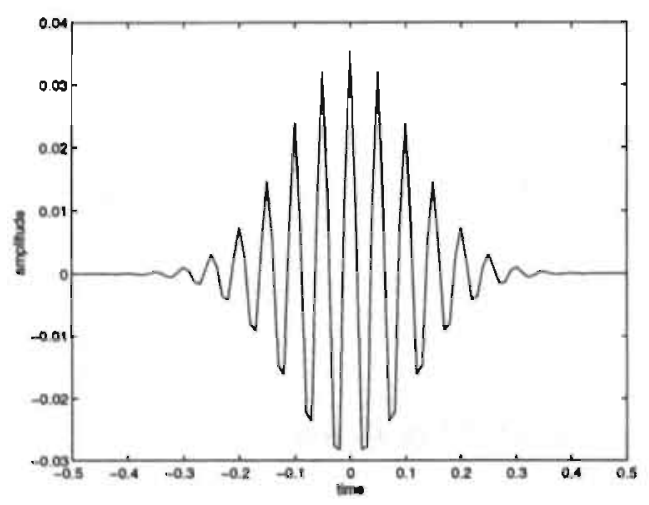

(c)

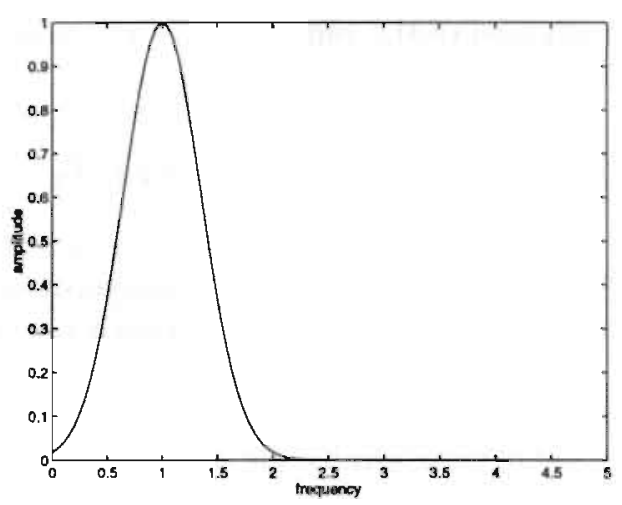

(b)

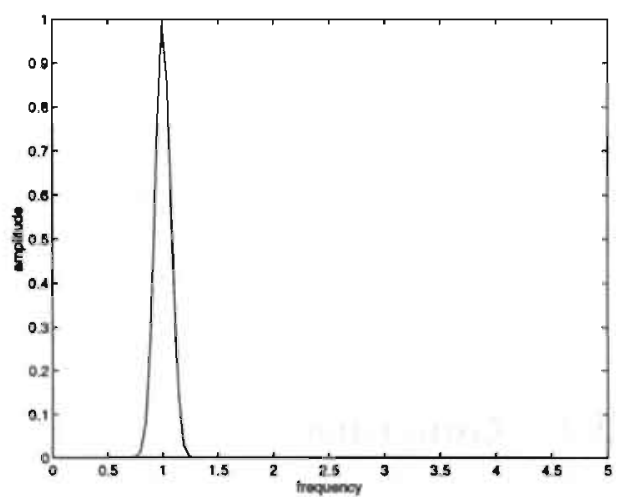

(d)

FIGURE 2.1. Two different pulse shapes varying in duration (left), together with the corresponding spectra (right). The width of the spectrum increases when the pulse is made shorter. (a), (b) correspond to $Q_{\mathrm{tr}}=2 ;$ (c), (d) to $Q_{\mathrm{tr}}=10 . Q_{\mathrm{tr}}$ determines the number of periods in time domain. 


\subsubsection{Ultrasonic Transducer}

The ultrasonic transmitter-receiver called the transducer is responsible for the generation of the ultrasonic beam and the detection of returning echoes. It is an electromechanical device converting electrical energy into acoustical energy and vice versa, because, as we have already mentioned, its central part is the piezoelectric element. Several transducer factors or parameters influence the overall performance of the transducer. We will mention just one, the coefficient $Q_{\mathrm{tr}}$, which characterizes the frequency response of the transducer. There, the subscript " $t r$ " refers to transducer. The $Q_{\text {tr }}$ value is a point of major consideration when modelling the returned signal, as we shall see later.

When the center frequency, $f_{\mathrm{tr}}$, is the primary operating frequency of the transducer, and $B_{\mathrm{tr}}$ is the bandwidth at $3 \mathrm{~dB}$ (which corresponds to the width at $70 \%$ of the peak power value) on either side of the center frequency, $f_{\mathrm{tr}}$, the $Q_{\mathrm{tr}}$ value is determined from

$$
Q_{\mathrm{tr}}=\frac{f_{\mathrm{tr}}}{B_{\mathrm{tr}}}
$$

The $Q_{\mathrm{tr}}$ value relates the damping characteristics to the pulse length as follows. A high $Q_{\mathrm{tr}}$ transducer has a. narrow bandwidth (Fig. 2.1.(d)), thus produces a long pulse length (hard to damp, Fig. 2.1.(c)). Whereas a low $Q_{\mathrm{tr}}$ transducer has a broad bandwidth (Fig. 2.1.(b)) and a short pulse length (easily damped, Fig. 2.1.(c)). In other words, according to [32], the $Q_{\mathrm{tr}}$ value reflects the number of periods in the pulse, $N_{\mathrm{p}}$, thus

$$
N_{\mathrm{p}}=Q_{\mathrm{tr}} .
$$

The number of periods, $N_{\mathrm{p}}$, corresponding to a signal of duration $t_{x}$, equals $N_{\mathrm{p}}=$ $t_{x} / T$, for a period at carrier frequency $T=\lambda / c$. The relationship given by (2.3.2) is also illustrated in figures 2.1.(a), and (c). A pulse that correspond to $Q_{\mathrm{tr}}=2$ contains two periods, another one contains ten cycles which is in accordance with $Q_{\mathrm{tr}}=10$. We see that the width of the spectrum increases when the pulse is made shorter.

It should be emphasized that $Q_{\mathrm{tr}}$ is a good specification of the performance of the transducer and is roughly of the same order despite its central frequency. Therefore, changing the center frequency, $f_{\mathrm{tr}}$, will not modify the pulse length in periods because the bandwidth will change to the same extent.

\subsubsection{Sound Beam and Resolution}

The resolution (or fineness of detail) refers to the system's ability to distinguish the echoes from two closely (in either direction within the plane of obscrvation) positioned reflectors in the tissue. This is influenced not only by how close the reflectors are positioned relative to each other (relative amplitude), but also by the sound beam and echo apparatus parameters. The relationship between the resolution and sound beam will now be discussed. 
The axial resolution is the ability of the system to separate structures lying closely along the propagation direction of the sound wave: the main axis. It is determined by the length of the ultrasound pulse generated by the transducer. Obviously, for shorter pulse length, the axial resolution is improved. In the previous section we have seen that the pulse length depends on the $Q_{\mathrm{tr}}$ value of the transducer. A lower $Q_{\mathrm{tr}}$ transducer produces a shorter pulse length (compare Figs. 2.1.(a),(c) for $Q_{\mathrm{tr}}=2$, and $Q_{\mathrm{tr}}=10$, respectively) and therefore, improves axial resolution. Short signals (low $Q_{\mathrm{tr}}$ ) represent, in fact, a wide range of frequencies, or a wide frequency spectrum, as shown in Fig. 2.1.(b). Hence, a good axial resolution can be achieved using short ultrasound bursts (Fig. 2.1.(a)), rather than continuous sound at an almost single frequency (Fig. 2.1.(c)).

The lateral resolution is the ability of the system to separate structures in a plane perpendicular to the main axis. It depends on the shape of the ultrasound beam produced by the transducer, e.g. by focusing. Decreasing the beam width will improve the lateral resolution, since a narrow beam enables small objects to become distinguishable. All systems should be designed to produce narrow beams, since this is usually the major limitation on resolution.

The second objective is to produce a beam of uniform intensity. In practice, a nonuniform intense beam exists in the near field (area of nondivergence) and a uniform beam is present in the far field (area of diverging beam in which lateral resolution is poor). It is possible to smooth the near field intensity pattern so as to yield a narrow beam with almost uniform intensity. A low $Q_{\mathrm{tr}}$ transducer (broad bandwidth and short pulse length) produces many frequencies rather than just a single frequency. The presence of multiple frequencies tends to make the near field more uniform. Each frequency has a different interference pattern, and when these patterns are superimposed on one another, the result is a smoothing of the overall intensity pattern.

In summary, the ideal situation for diagnostic ultrasound would be to have only a single cycle instead of several cycles due to axial resolution aspects. Therefore, diagnostic ultrasound utilizes low $Q_{\mathrm{tr}}$ transducers, activated by a short pulse resulting in the generation of the impulse response. In practice, the $Q_{\mathrm{tr}}$ value is restricted by the bandlimited character of the transducer, the lowest $Q_{\mathrm{tr}}$ value will be around 2 .

\subsubsection{Signal Visualization}

During echo reception the piezoelectric element converts the received sound pulse into an electrical signal, or often called the radio frequency (RF) signal. It is this signal that is used for further processing or creating the echo display. Various techniques have been used to display echo-signal data in graphic and image formats: A-mode, B-mode, and M-mode display.

An A-mode scanning refers to amplitude-mode scanning. The term amplitude corresponds to the strength of the detected echo signal (envelope). A-mode displays the amplitude of the signal in one dimension versus the depth or time (delay) of the 
signal in the other dimension. Depth and time are interchangeable by (2.2.4). The A-line represents a great amount of information concerning various tissue interfaces in the body. B-mode uses the brightness of a dot to indicate the amplitude of the signal, as a function of depth; M-mode is a special scanning technique that plots the motion of the $\mathrm{B}$-mode dots with respect to time. Two-dimensional (2D) visualization is an extension of $\mathrm{B}$-mode where the ultrasound beam position or direction is varied from emission to emission to scan the plane of observation.

\subsection{Physical Properties of the Pulsed RF Signal}

This section is dedicated to the features of the data from the pulsed wave instrument with a fixed beam position. The returned signals from sequential pulses are acquired and aligned with one another to form function $e(x, t)$, shown in Fig. 2.2. The $x$ variable represents the depth of the measurement and the $t$ variable represents time in multiple of the pulse repetition period, $T_{\text {prf }}$. One particular signal at a fixed instant, e.g. the return due to the $k$-th pulse, is a (continuous) function of deptli, $e(x, k)$. The collection of points at fixed depth, $x_{1}$, denoted as $e\left(x_{1}, t\right)$ is separated by pulse repetition period $T_{\text {prf }}$ and is thus a (discrete) function of time.

In the following subsections 2.4.1 to 2.4 .3 the properties of a pulsed radio frequency $(\mathrm{RF})$ signals will be described as a function of both variables simultaneously, and also as a function of one variable while the other one will be fixed. Aliasing effects in depth and velocity are subject of Section 2.4.4. The noise sources and signal-to-noise ratio will be covered in Section 2.4.5.

\subsubsection{Spatial and Temporal Properties of the RF Signals}

In this section the characteristics of successive RF signals are studied, which are separated by pulse repetition period $T_{\mathrm{prf}}$ as shown in Fig. 2.2, thus signal $e(x, t)$ as a function of both variables: depth and time is of interest.

In the case of blood, the backscattered signal is mainly due to aggregates of red cells. Although these aggregates are of a generally time-varying nature (because of the pulsatility of blood flow), nevertheless, they are temporary stationary that permits the ultrasonic measurement of blood flow. The blood flow can be considered as stationary no longer than 5 to $10 \mathrm{~ms}$ [25]. For a pulse repetition period $T_{\text {prf }}$ in the order of $0.1 \mathrm{~ms}$ it indicates that there exists a time-invariant velocity field over a number of subsequent RF signals.

Let us assume that scatterers within the sample volume are all moving with the same velocity and the angle between the beam and velocity directions is $\theta$. Due to the changing distance between the transducer and groups of moving scatterers, the backscattered signal exhibits the gradual translation with respect to the previous pulse returns (illustrated in Fig. 2.2). It means that. RF return due to the pulse $k$, $e(x, k)$, is just a shifted version of the RF return due to pulse $k-1, e(x, k-1)$. Hence, 


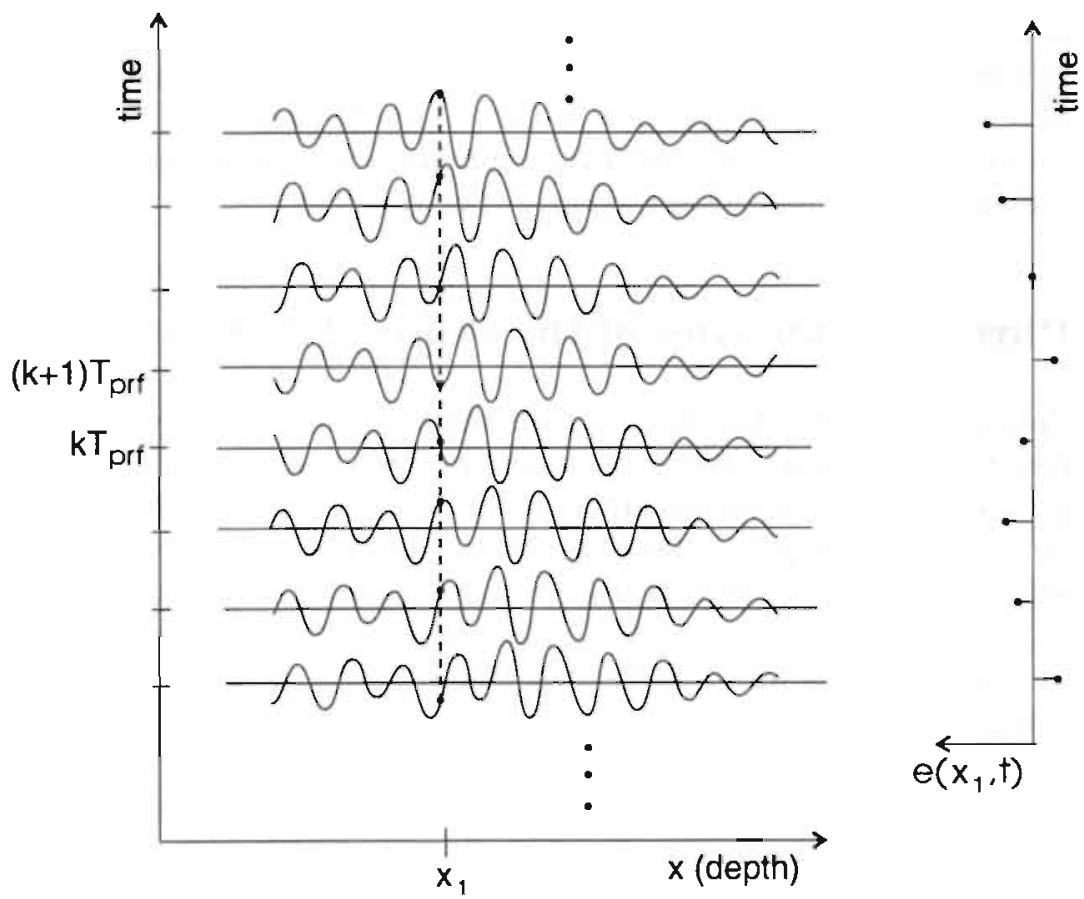

FIGURE 2.2. Representation of the pulsed data obtained from sample volume as a function of variables $x$ (depth in tissue) and time. $T_{\mathrm{pr} f}$ is a pulse repetition period. Pulsed Doppler signal, $e\left(x_{1}, t\right)$, is a signal (as a function of time) intersected at $x_{1}$.

the signal due to the $k$-th transmission can be written as

$$
e(x, k)=e\left(x+x_{\tau}, k-1\right)
$$

where $x_{r}$ is the displacement that the scatterers (travelling at velocity $v \cos \theta$ in the direction of the beam) have moved between two excitations, thus

$$
r_{r}=v \cos \theta T_{\text {prf }} .
$$

This time shift gives the translation of the successive echoes induced by scatterers displacements between the excitations. The time delay $\tau$ that corresponds to the displacement $x_{\tau}$ is by (2.2.4) equal to

$$
\tau=\frac{2 x_{\tau}}{c}=2 \frac{v \cos \theta}{c} T_{\text {prf }}
$$

This concept was developed for the blood velocity estimation by Foster in [23]. The same expression for time delay $\tau$ was derived also by Bonnefous in [8]. 
In summary, scatterers moving at the same velocity will induce a signal with gradually changing phase and amplitude characteristics within the sample volume over subsequent $\mathrm{RF}$ lines. If the scatterers within the sample volume are not moving at the same speed or do not follow the same path through the sample volume, the similarity of the signals will degrade.

\section{Spatial and Temporal Spectral Properties of the RF Signals}

This part includes the application of the Fourier transform on the pulsed RF data, $e(x, t)$, which is a function of depth in tissue, $x$, and time, $t$. Their frequency counterparts are $\xi$, whose dimension is length ${ }^{-1}$, and $f$ whose dimension is $\operatorname{time}^{-1}$ respectively. The variable $\xi$ is proportional to the frequency of the received RF signal (spatial frequency), and $f$ can be identified with the Doppler frequency shift (temporal frequency). Spatial frequency can be converted into units of time $e^{-1}$ (following the relationship 2.2 .4 ) as

$$
f=\frac{1}{2} c \xi
$$

Let us consider the idealized continuous received signal $e(x, t)$ where both $x$, and $t$ are unwindowed and infinite in extent. In [71] it was shown that the Fourier transform of idealized data from moving scatterers, $E(\xi, f)$ is a line whose slope is the velocity of the scatterers.

Replacement of the infinite, continuous assumption with a finite, sampled signal transforms the spectrum $E(\xi, f)$ into a function of finite width. This process is a manifestation of spectral broadening due to the limited extent of data. In [44] it was shown that despite this spectral broadening the feature of having the concentration of nonzero frequency components around a straight line passing through the origin in $\xi-f$ plane will be retained. A spectrum will consist of two smeared line segments centred at plus/minus the central frequency of $\left[\xi_{\mathrm{rf}}, f_{\mathrm{d}}\right]$ and having slope of

$$
f=(v \cos \theta) \xi, \quad \xi \in \mathbf{R}, f \in \mathbf{R},
$$

where $v \cos \theta$ is the component of velocity in the direction of the ultrasound beam. Therefore, any spectral component in the $\xi$ dimension has a corresponding component in the $f$ dimension. This observation can be covered by the relationship between the spatial quality factor, $Q_{\mathrm{rf}}$ (the ratio of spatial centre frequency, $\xi_{\mathrm{rf}}$, and spatial bandwidth, $B_{\mathrm{rf}}$ ), and the temporal quality factor, $Q_{\mathrm{d}}$ (the ratio of temporal centre frequency, $f_{\mathrm{d}}$, and temporal bandwidth, $B_{\mathrm{d}}$ )

$$
Q_{\mathrm{rf}}=Q_{\mathrm{d}}
$$

which means that the proportional spatial spectral properties of the RF' signal (at fixed time) are the same as the temporal. Into a relation given by equation (2.4.5) a particular frequency can be substituted. Namely, the temporal center frequency, $f_{\mathrm{d}}$, can be expressed as a function of the spatial center frequency, $\xi_{\mathrm{r}}$, as

$$
f_{\mathrm{d}}=(v \cos \theta) \xi_{\mathrm{rf}},
$$




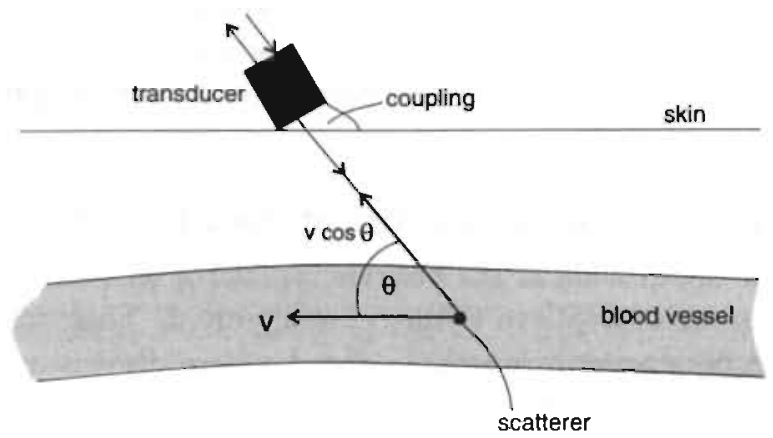

Figure 2.3. Blood flow measurement.

or using (2.4.4) as

$$
f_{\mathrm{d}}=\left(2 \frac{v \cos \theta}{c}\right) f_{\mathrm{rf}} .
$$

This equation is a general formulation of the Doppler equation. The choice of frequency of RF signal, $f_{\mathrm{rf}}$, in this expression is crucial for various processing methods of velocity estimation which will be explored in Chapter 3 .

\subsubsection{Temporal Properties of the RF Signals}

In this section attention is paid to the signal obtained from the set of received signals $e(x, t)$ when $x$ is fixed, for instance at $x_{1}$, and $t$ is variable, $e\left(x_{1}, t\right)$. It corresponds to a vertical "slice" through the ensemble at depth $x_{1}$ as illustrated in figure 2.2. This signal, $e\left(x_{1}, t\right)$ is just a function of time and is therefore a (discrete) version of the Doppler signal (sampled at period $T_{\mathrm{prf}}$ ) originating from the sample volume located at depth $x_{1}$.

By the Doppler sigual we mean the audio signal produced by a conventional continuous wave system (CW). It is the simplest velocity detection instrument, where in the transducer, two elements operate continuously: one for transmission and one for reception. For signal processing a continuous signal is available.

Consider the ultrasonic transducer transmitting waves of frequency $f_{e}$ which is positioned on the body surface so that the ultrasound beam strikes the blood vessel at an angle $\theta$ (see Fig. 2.3). The beam propagates through the body, where the wave velocity is $c$. The beam is scattered by particles of blood, which have a velocity $v$ relative to the vessel, but which have $\operatorname{component} v \cos \theta$ in the direction of the beam. Then by a well known phenomenon from physics, the Doppler effect, any moving structure or surface in the body acting as a scatterer causes a Doppler frequency shift, $f_{\mathrm{d}}$, which is a difference between transmitted and received frequencies. The 
received frequency is changed by an amount proportional to the velocity at which the scattering centers are moving towards or away from the transducer. The Doppler shift is defined by the Doppler equation

$$
f_{\mathrm{d}}=2 \frac{v \cos \theta}{c} f_{\mathrm{e}} .
$$

This equation is exactly the same as (2.4.8) assuming that frequency of the RF signal, $f_{\mathrm{rf}}$, equals the emitted frequency, $f_{\mathrm{e}}$. Since the frequency change, $f_{\mathrm{d}}$, can be detected by suitable electronic circuits, the velocity $v$ can be calculated. With this principle very high velocities can be measured. It is, however, not possible to know the exact position along the beam from which the measurement was obtained. This is termed range ambiguity, and is the major drawback to the continuous wave technique.

The pulsed system is an ingenious modification of the continuous system that allows range discrimination. In pulsed wave system ultrasound pulses are transmitted with pulse repetition frequency $f_{\mathrm{prf}}$, or in other words, time between successive pulses is $T_{\text {prf }}=f_{\text {prf }}{ }^{-1}$. Between transmissions, the transducer is switched to reception mode. To obtain signals from a selected position, for instance, $x_{1}$, a delay after transmission of ultrasound pulse has to be introduced. This delay is defined by the length of time necessary for the sound wave to travel to the point of interest and be scattered back to the transducer (it follows from equation 2.2.4).

Let us assume that in the vicinity of $x_{1}$ the received RF signal has a sinusoidal shape with instantaneous frequency $f_{\mathrm{rf}}$ and with an arbitrary amplitude. Then, due to the motion of scatterers, the phase of the received signal at position $x_{1}$ will vary over subsequent observations (Fig. 2.2). A complete cycle will be covered if the scatterers are displaced over a distance $d$ equal to

$$
d=\frac{\lambda}{2}=\frac{c}{2 f_{\mathrm{rf}}} .
$$

The time, $t_{\mathrm{d}}$, that it takes to travel the distance $d$, depends on the velocity of the scatterers along the ultrasound beam, thus

$$
t_{\mathrm{d}}=\frac{d}{v \cos \theta}=\frac{c}{2 \hat{f}_{\mathrm{rf}} v \cos \theta} .
$$

For a pure harmonic RF signal with carrier frequency $f_{\mathrm{rf}}$ the signal at $x_{1}$ will have also a single frequency, $f_{\mathrm{d}}$,

$$
f_{\mathrm{d}}=\frac{1}{t_{\mathrm{d}}}=2 \frac{v \cos \theta}{c} f_{\mathrm{rf}},
$$

which is again the familiar Doppler expression but now for a pulsed Doppler instrument. This equation is identical to (2.4.8), here it derived in a different manner. 


\section{Temporal Spectral Properties of the RF Signals}

To study the spectral properties of the pulsed RF signal as a function of the temporal frequency $f$, we will consider an intersection of the two dimensional spectral amplitude distribution $E(\xi, f)$ at a spatial frequency $\xi_{1}$, yielding $E\left(\xi_{1}, f\right)$. At this cross-section the amplitude is nonzero for a finite interval of temporal frequencies. It was stated in Section 2.4.1 that the spectral content in $\xi-f$ plane is concentrated around a line passing through the origin. Because of this linearity, the spectral characteristics at spatial frequency $\xi_{1}$ are covered by the temporal quality factor $Q_{\mathrm{d}}$ defined in Section 2.4.1. $Q_{\mathrm{d}}$ is finite at fixed spatial frequency $\xi_{1}$. Thus, despite of the relationship given by (2.4.12) even a single scatterer travelling along the axis of an ultrasound beam would not be transformed to a single temporal frequency. A single frequency would cause $Q_{\mathrm{d}}=\infty$. The effect that a single velocity induces a band of frequencies is due to the transit time effect that was first studied by Newhouse [51]. Transit time is the time required for a scatterer to pass through the sample volume. Because the sample volume is of finite size the scatterer contributes to the signal only during the limited time that yields the spectral broadening.

It should be noted that the temporal quality factor, $Q_{\mathrm{d}}$, is independent of velocity. Because of proportionality between velocity and frequency given by $(2.4 .12)$, an increase of the velocity increases the center frequency $f_{\mathrm{d}}$ by the same factor. However, $Q_{\mathrm{d}}$ is independent of $f_{\mathrm{d}}$ which was already emphasized in Section 2.3.1. As a result, the temporal quality factor, $Q_{\mathrm{d}}$, does not depend on the velocity.

\subsubsection{Spatial Properties of the RF Signals}

The subject of this section is the RF signal at fixed time, thus just a line of sight that can be seen in figure 2.2 for example at time $t=k T_{\text {prf }}$.

In Section 2.2 .3 we have already mentioned that the backscattered signal is composed of the sum of the signals returned by a large number of randomly located scatterers. Generally, the scattered sound waves from different particles can interfere with one another constructively and destructively, so the signal from a large number of uncorrelated scatterers depends on the relative positions. The RF signal, induced in the transducer by the backscattered echoes (at given instant), is an amplitude versus depth signal (like one line of sight in Fig. 2.2), possibly with some distortion from the propagating medium.

\section{Spatial Spectral Properties of the RF Signals}

The spectral properties of the pulsed RF signal as a function of spatial frequency $\xi$, will be considered at an intersection of the two dimensional spectral amplitude distribution, $E(\xi, f)$, at a temporal frequency $f_{1}$, yielding $E\left(\xi, f_{1}\right)$. At this cross-section various spatial frequency components are identified. Following the same reasoning as in Section 2.4.2 the spatial spectral characteristics are covered by a spatial quality factor $Q_{\mathrm{rf}}$ defined in Section 2.4.1. Under the assumption that there is no frequencydependent attenuation and that the transducer is only activated with a Dirac im- 
pulse, the spatial quality factor, $Q_{\mathrm{rf}}$, equals the quality factor of the transducer, $Q_{\mathrm{tr}}$ (defined by (2.3.1) for $f_{\mathrm{tr}}=f_{\mathrm{rf}}$ and using relation 2.4.4),

$$
Q_{\mathrm{rf}}=Q_{\mathrm{tr}}
$$

The spatial center frequency of the RF signal, $\xi_{\mathrm{rf}}$, is however a function of depth due to the frequency-dependent attenuation of the ultrasound in tissue and frequencydependent scattering as was already mentioned in Sections 2.2.2, and 2.2.3. Previous research [16] has shown that intervening tissue acts as a low pass filter for the transmitted waveform, and that the scattering response of the cells acts as a high pass filter. These effects do not cancel each other because attenuation by intervening tissue has a stronger influence on frequency with increasing depth. It was shown by Ferrara et al. [20] that a major effect of frequency-dependent attenuation is a shift of the center frequency to lower frequencies.

It should be noted that due to the depth-dependent attenuation, which affects higher frequencies more than lower frequencies, the shape of the spatial frequency distribution will be a function of depth, especially for wideband RF signals.

\subsubsection{Pulse Repetition Frequency and Aliasing}

As previously stated, a pulsed RF signal transforms into a sampled version of a conventional Doppler signal when depth is fixed at some particular value. In pulsed wave systems ultrasound pulses are transmitted with pulse repetition frequency, $f_{\mathrm{prf}}$. Thus at the rate of $T_{\mathrm{prf}}=f_{\mathrm{prf}}{ }^{-1}$, the associated returned signals are received, the continuous Doppler signal is sampled and therefore, $f_{\text {prf }}$ may be seen as a temporal sampling frequency.

Since the time between subsequent emitted pulses is determined by the depth of the point of interest, the pulse repetition frequency is limited by the depth of that point: emission of the next burst is only allowed if the returned signal of the previous burst has been received, otherwise aliasing in depth will occur. The largest depth $d_{\max }$ into the body that can be investigated uniquely is therefore:

$$
d_{\max }=\frac{1}{2} \frac{c}{f_{\mathrm{prf}}},
$$

where $c$ is the propagation velocity. For example, to investigate a depth of $4 \mathrm{~cm}$, the maximum pulse repetition frequency will be of the order of $19 \mathrm{kHz}$.

It has been mentioned that in pulsed systems the transducer switches between transmission and reception, and the switching frequency is known as pulse repetition frequency. The frequency shift (e.g. velocity) of signals selected from a given depth has to be reconstructed from sampled information (with the sampling rate $f_{\text {prf }}$ ). The Nyquist theorem states that it is only possible to reconstruct a time signal accurately from samples if the sampling frequency is twice as high as the highest frequency component in the signal. Thus if the sampling rate is $f_{\mathrm{prf}}$, but the Doppler signal is in 
excess of $f_{\mathrm{pr}} / 2$, the correct relationship between the reconstructed signal and the original is lost. As a result, only velocity of limited magnitude can be measured. When the Doppler frequency shift exceeds a limit defined by the Nyquist theorem (which equals $f_{\text {prf }} / 2$ ), distortion of the signal takes place whereby the signal wraps upon itself and appears to artificially change direction. Velocities higher than those related to Nyquist limit reappear as other, incorrect, velocities on the display. This phenomenon is known as velocity aliasing.

The peak velocity that can be correctly resolved by the pulsed Doppler system is limited by the choice of the pulse repetition frequency $f_{\text {prf }}$, and therefore by the depth of the point of interest. In the beginning of this section we have seen that the depth of the target dictates a maximum $f_{\mathrm{prf}}$. The greater the depth, the lower the pulse repetition frequency. It means also lower velocity at which aliasing occurs and therefore, lower maximum measurable velocity. The maximum velocity which can unambiguously be measured $v_{\max }$, may be found by substituting (2.4.9) into (2.4.14), i.e.

$$
v_{\max }=\frac{c f_{\mathrm{pr} f}}{4 \int_{\mathrm{e}} \cos \theta}
$$

where $f_{e}$ is the frequency of the transmitted signal, $c$ is ultrasound speed, and $\theta$ is an angle between the direction of ultrasound beam and the velocity. A combination of equations (2.4.14) and (2.4.15) results in

$$
v_{\max }=\frac{c^{2}}{8 d_{\max } f_{\mathrm{e}} \cos \theta},
$$

and provides a relationship between the maximum detectable velocity at the maximum observable depth.

\subsubsection{Noise Sources and Signal-to-Noise Ratio}

The RF signal is obscured because of variability in the signal, which may stem from various sources. One source of interference is the surrounding moving structures adjacent to the studied flow or located along the acoustic path. These structures will induce, like red cells, a Doppler effect. For instance, the vascular walls, or the heart wall are involved. The signal generated in that way is very strong with respect to the useful signal since the structures that have generated it are strongly reflective in comparison with the red cells. On the other hand, the corresponding Doppler frequencies are relatively low, since the speed of displacement of these structures is low with respect to that of red cells. This type of noise, however can be removed by appropriate filtering. Filters known as wall filters are required to remove constant and low-frequency components of the pulsed Doppler signal. In this thesis we will not go into the details of low frequency clutter filtering. A comprehensive evaluation of the effects of echo suppression on velocity estimation can be found in [10]. From now on we will not consider the low-pass filtering operation, we will assume that the backscattered signal is not affected by surrounding structures. Of course, this assumption does not restrict the applicability of the study. 
The variation in measurement is also due to fluctuations in the measurement process itself rather than in the value of the quantity being measured (which is temporary stationary as explained in Section 2.4.1). The reasons for this variability include noise, such as thermal, background and measurement noise, commonly termed instrumentation noise. Its contribution is not limited only to certain frequencies, rather it is uniformly distributed over the entire frequency range, $-f_{\mathrm{prf} / 2}, f_{\mathrm{prf} / 2}$. This is because the noise is independent of the time of observation introducing a random phase in the received $\mathrm{RF}$ signal. Thus, provided low-frequency clutter has been removed, only the instrumentation noise is of importance in the evaluation of blood velocity measurements.

The signal-to-noise ratio, SNR, is a useful figure of merit for characterizing the measurement system. It is defined as the ratio of the power of the signal $e(x, t)$ to the power of the noise signal $n(x, t)$

$$
\mathrm{SNR}_{\text {ratio }}=\frac{\int_{-\infty}^{\infty} \int_{-\infty}^{\infty} e^{2}(x, t) d x d t}{\int_{-\infty}^{\infty} \int_{-\infty}^{\infty} n^{2}(x, t) d x d t}=\frac{\int_{-\infty}^{\infty} \int_{-\infty}^{\infty}|E(\xi, f)|^{2} d \xi d f}{\int_{-\infty}^{\infty} \int_{-\infty}^{\infty}|N(\xi, f)|^{2} d \xi d f}
$$

where $|E(\xi, f)|^{2}$ is a power density spectrum of the signal $e(x, t)$ and $|N(\xi, f)|^{2}$ is a power density spectrum of the noise $n(x, t)$ because power results through its integration [52]. Relative measurements are usually made and given in decibels (dB). A decibel is defined as

$$
\mathrm{dB}=10 \log _{10} \frac{I}{I_{0}},
$$

where $I$ is the intensity at the area of interest, and $I_{0}$ is the original or reference intensity. The use of decibels allows one to express a wide range of any variable, including intensity, amplitude, power, noise levels, etc. The SNR $\mathrm{R}_{\text {ratio }}$ for systems is also often stated in terms of decibels $(\mathrm{dB})$. Specifically,

$$
\mathrm{SNR}_{\mathrm{dB}}=10 \log _{10} \mathrm{SNR}_{\text {ratio }}
$$

Further, in this thesis, we will skip the subscript $\mathrm{dB}$, or ratio. It will be straightforward, since the dimensionless number will correspond to $\mathrm{SNR}_{\mathrm{ratio}}$, and $\mathrm{SNR}_{\mathrm{dB}}$ will be stated with $\mathrm{dB}$.

\subsection{Summary}

In this chapter we have presented a survey of some physical notions, principles and instruments associated with ultrasonic measurements of blood flow velocity. We have discussed some basic physics like the propagation phenomenon, the echo principle, the interaction of ultrasonic wave and blood. We have explained why the quality factor $Q_{\mathrm{tr}}$ is the most important characteristic of the ultrasonic transducer. The transducer is responsible for generation of the ultrasound beam and detection of 
returning echoes. We have shown that for a good axial and lateral resolution a low $Q_{\text {tr }}$ transducer is desirable.

Further, we have examined the physical properties of the pulsed backscattered signals, also called radio frequency (RF) signals. We have seen (Fig. 2.2) that one particular return due to the $\mathrm{k}$-th transmitted pulse is a function of depth. The subsequent return is separated by the pulse repetition period $T_{\text {prf }}$. Due to the changing distance between the transducer and groups of moving scatterers the subsequent signals exhibit gradual translation with respect to the previous signal. It is important. to recognize that the change in phase (distance) between successive returns carries information about the velocity of moving scatterers. The received signal due to one pulse does not contain information about the velocity of the scatterers.

After we have recognized what physical quantity carries the desired information, the next step is to extract this information by processing the backscattered signals. The principles of signal processing for blood velocity estimation are going to be discussed in the following chapter. 


\section{Chapter 3}

\section{Principles of Signal Processing for Blood Velocity Estimation}

\subsection{Introduction}

The techniques of signal processing have as their main objective the separation of information which is of use from that which is not. They make use of the redundancy in the raw data to reduce the effects of noise and confounding mechanisms which are of no interest to the observer. In case of blood velocity measurement, the processing of ultrasound signals may be described in terms of three stages:

- measurement, where RF signals are captured, aligned and recorded.

- signal transformation, where known properties of the signals described in the previous chapter are used to reduce the variability and facilitate analysis. It includes sampling of received signals and filtering of the raw data to remove low-frequency clutter or phase detection (demodulation) to shift the contents of the RF spectrum towards zero.

- feature extraction and interpretation, where key features of the transformed signal are extracted to make a physiological interpretation and diagnostic classification. This involves mainly the estimation of the (spectral) characteristics of the signal (velocity, velocity width) which is based on the physical properties of the pulsed RF signal described in section 2.4. The basic physical principle in blood velocity estimation is the gradual translation of the backscattered signal with respect to previous returns, due to the changing distance between the transducer and groups of moving scatterers.

The objective of this chapter is to introduce some matters associated with the processing of ultrasound signals for blood velocity estimation, and to review the statistical principles pertinent to our analysis in latter chapters. First, in Section 3.2 the form of the data is described. Then, in Section 3.3 some statistical notions necessary for this study are reviewed. In Section 3.4 a common signal transformation, preliminary to some processing techniques is summarized. The main processing concepts of blood velocity estimation including the processing of low frequency as well as high frequency signals are the subject of Section 3.5. 


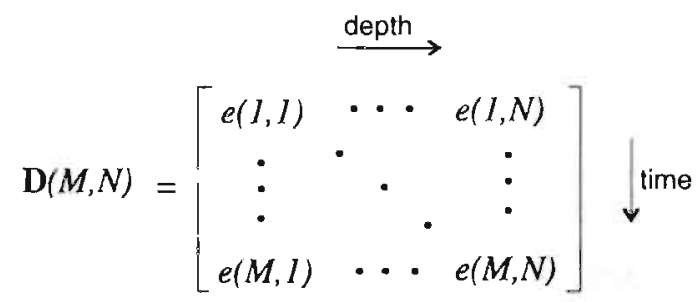

FIGURE 3.1. Representation of data in matrix form.

\subsection{Form of the Data}

We now return to Section 2.4, namely the representation of the pulsed data that is a function of depth and time as illustrated in figure 2.2. The data consist of a continuous record of $e(x, k)$ over depth. However, if we wish to analyse such a record by digital methods we would have to read off its values at small intervals, the sampling distance $x_{\mathrm{s}}$ (subscript " $\mathrm{s}$ " refers to the sampled notions). It is assumed that $x_{\mathrm{s}}$ is chosen sufficiently small to avoid aliasing effects. In other words, the spatial sampling frequency, $\xi_{\mathrm{s}}=x_{\mathrm{s}}^{-1}$, is chosen so that it complies with the Nyquist bound (twice the anticipated maximal frequency of the RF signal). Because $Q_{\mathrm{rf}}>1$ (see a discussion in Section 2.4.3) it follows that the maximal frequency would not be higher than $2 \xi_{\mathrm{rf}}$, where $\xi_{\mathrm{rf}}$ is the spatial carrier frequency. A spatial sampling frequency

$$
\xi_{5}=4 \xi_{\mathrm{rf}}
$$

would be adequate for most applications. This sampling operation causes the spectrum of the signal to be replaced by multiple copies at intervals of $\xi_{\mathrm{s}}$ in the $\xi$ direction. Likely intrinsic temporal sampling by pulse repetition frequency, $f_{\mathrm{prf}}$, yields the repetition of the spectra at intervals of $f_{\text {prf }}$ in the $f$ direction. Because the spectrum is periodic we only have to consider the fundamental frequency plane taken as $-\xi_{\mathrm{s}} / 2 \leq \xi<\xi_{\mathrm{s}} / 2,-f_{\mathrm{prf}} / 2 \leq f<f_{\mathrm{prf}} / 2$.

If we make use of the normalized frequencies in terms of temporal and spatial sampling rates (i. e., $\Psi=\xi x_{s}$ and $F=f\left(T_{\text {prf }}\right.$ ) we can write the relationship (2.4.5) as

$$
F=\left(v \cos \theta \frac{\xi_{\mathrm{s}}}{f_{\mathrm{prf}}}\right) \Psi
$$

where $v \cos \theta$ is the component of velocity in the direction of the ultrasound beam. For the normalized frequencies $(\Psi, F)$ the fundamental plane extends from $-1 / 2 \leq$ $\Psi<1 / 2,-1 / 2 \leq F<1 / 2$. When we assign the entity between the brackets as $\varphi$, the dimensionless velocity, we can rewrite (3.2.2) as follows

$$
F=\varphi \Psi
$$


The dimensionless velocity, $\varphi$, can be converted into units of $\mathrm{ms}^{-1}$ by

$$
v=\varphi \frac{f_{\mathrm{prf}}}{\xi_{\mathrm{s}}} \frac{1}{\cos \theta}=\varphi \frac{f_{\mathrm{prf}}}{f_{\mathrm{s}}} \frac{c}{2 \cos \theta},
$$

where equation (2.4.4) was substituted for $\xi_{\mathrm{s}}$, where $c$ is acoustic velocity.

The discrete data consists of the values of the process recorded over a finite segment in depth and time forming a matrix $\mathbf{D}(M, N)$, where the first parameter $(M)$ specifies the row number (time) whereas the second parameter $(N)$ specifies the column number (position). These parameters depend on temporal and spatial dimensions of a data window. The size of the window in the temporal direction is defined by a number of considered subsequent observations. For example, $e(x, k)$ for $k=1, \ldots, M$ result in $\mathrm{M}$ rows of matrix $\mathrm{D}(M, N)$. The spatial dimension of the window is usually given by a number of periods $N_{\mathrm{p}}$ at carrier frequency $\xi_{\mathrm{r} f}$. One period, $N_{\mathrm{p}}$, represents $\xi_{\mathrm{s}} / \xi_{\mathrm{rf}}$ sample points in depth. Therefore, number of samples in depth, $N=N_{\mathrm{p}} \xi_{\mathrm{s}} / \xi_{\mathrm{rf}}$. The final representation of data in matrix form is illustrated in figure 3.1.

\subsection{Related Statistical Principles}

The objective of this section is to review some of the elements of statistical theory which are essential for our subsequent study. For this purpose we first define the expectation operator. The usefulness of the expectation notation lies in the fact that we may write the definitions in a more compact form.

For any random variable $X$, we define the expected value of $X$, written as $\mathcal{E}[X]$, by

$$
\mathcal{E}[X]= \begin{cases}\sum_{i} x_{i} P\left(x_{i}\right), & \text { if } X \text { is discrete } \\ \int_{-\infty}^{\infty} x p(x) d x, & \text { if } X \text { is continuous, }\end{cases}
$$

provided that the above sum or integral exists, and $X$ has some probability distribution: $P\left(x_{i}\right)$ is a discrete probability distribution, $p(x)$ is the probability density function. Thus, $\mathcal{E}[X]$ may be regarded as the "mean value of $X$ " in the sense that it is computed as a weighted average of all possible values of $X$, each value being weighted by the corresponding probability.

The material of this section includes various statistical principles. Section 3.3.1 de. scribes the concept of correlation which appears to be of great usefulness in blood velocity estimation. Section 3.3 .2 gatbers the definitions of the performance measures (bias, standard deviation) in statistical data evaluation. Section 3.3 .3 introduces the Cramér-Rao inequality and its link to estimation efficiency. 


\subsubsection{Correlation}

The title of this thesis reminds us of the fact that we will deal with the application of correlation to velocity estimation. The correlation function of data is most directly interpreted as a measure of the interrelationship between the observed data [5]. For instance, consider a sequence of RF signals in Fig. 2.2. How identical these signals are can be ascertained quantitatively with the correlation function. In this section we introduce a definition of correlation function as well as the estimator of correlation function and its statistical properties.

The correlation of two arbitrary random processes $X(t)$, and $Y(t)$, denoted by $\gamma(\tau)$, is defined by the relation

$$
\gamma(\tau)=\mathcal{E}\left[X^{*}(t) Y(t+\tau)\right],
$$

thereby assuming that the processes are stationary. In (3.3.2), the symbol * denotes the complex conjugate, so that, $X^{*}(t)=\operatorname{Re}[X(t)]-j \operatorname{Im}[X(t)]$. If $X(t)$ and $Y(t)$ are identical processes, equation (3.3.2), is usually called the auto-correlation function. If $X(t)$ and $Y(t)$ are different, the term cross-correlation is normally used.

The correlation function defined by equation (3.3.2) can be arrived at by more direct operations on the time history data representing the random processes $X(t)$ and $Y(t)$. For the special case of stationary (ergodic) data, the correlation function can be calculated from a single time history measurements $x(t)$ and $y(t)$ as follows:

$$
\gamma(\tau)=\int_{-\infty}^{\infty} x^{*}(t) y(t+\tau) d t, \quad \tau \in \mathbf{R} .
$$

Thus to perform correlation, $x(t)$ is slided by $y(t)$, and the product is integrated from $-\infty$ to $\infty$ for each value of time $t$. The discrete equivalent of equation (3.3.3) is defined as

$$
\gamma(n)=\sum_{n \in \mathbf{Z}} x^{*}\left(t_{i}\right) y\left(t_{i}+n\right), \quad n \in \mathbf{Z} .
$$

So far, the definition of correlation function has been presented in various forms. The next step is to provide the method of estimating correlation function on our data.

Given a data matrix $\mathbf{D}(M, N)$ as illustrated in Fig. 3.1, so that we have $N M$ samples $e(1,1), \ldots, e(M, N)$, suppose that we wish to determine their correlation $\gamma(n, m)$. A potential nonparametric estimator of correlation function is

$$
\begin{aligned}
\hat{\gamma}(m, n) & =\frac{1}{(N-|n|)(M-|m|)} \sum_{k=1}^{k=M-|m|} \sum_{\ell=1}^{\ell=N-|n|} e^{*}(k, \ell) e(k+m, \ell+n), \\
n & =0, \pm 1, \pm 2, \ldots,(N-1), \\
m & =0, \pm 1, \pm 2, \ldots,(M-1),
\end{aligned}
$$

with $\hat{\gamma}(m, n)=0$ for $|n| \geq N$ and $|m| \geq M$. It doesn't mean that the true $\gamma(m, n)=$ 0 for $|n| \geq N$ and $|m| \geq M$, but that we have arbitrarily chosen to set $\hat{\gamma}(m, n)=0$ 
for those values of $n$, and $m$ for which there are no nonzero products available. The estimator (3.3.5) averages all available pairs with depth and time lags between samples equal to $|n| T_{\text {prf }}$ and in the other dimension $|m| x_{\mathrm{s}}\left(T_{\mathrm{prf}}\right.$, and $x_{\mathrm{s}}$ are sampling ratios in time and depth).

The estimator (3.3.5) is unbiased according to Priestley [53]. It is expected that $\hat{\gamma}(n, m)$ will be a better estimate (in term of variance reduction) of $\gamma(n, m)$ for $n, m$ near zero than for large $n, m$ because more pair-products are averaged for small $n, m$ than for large $n, m$.

\subsubsection{Quality Measures in Data Evaluation}

In succeeding chapters we shall investigate the performance of estimation techniques by means of computer simulations also called the Monte Carlo method. The Monte Carlo method is according to Halton [24] generally defined as "representing the solution of a problem as a parameter of a hypothetical population, and using a random sequence of numbers to construct a sample of the population, from which statistical estimates of the population can be obtained". In our situation the parameter is obtained from a normally distributed collection of velocity estimates resulting from several independent realizations of our stochastic process (a data matrix of Fig. 3.1 is considered as one realization). The statistical estimates: sample mean and standard deviation describe then the properties of a population for a considered estimator.

The sample mean $\bar{v}$, and estimate of the standard deviation $\hat{\sigma}$ based upon $S$ independent estimates $\hat{v}_{i}$, for $i=1, \ldots, S$ can be obtained as follows:

$$
\begin{gathered}
\bar{v}=\frac{1}{S} \sum_{i=1}^{S} \hat{v}_{i}, \\
\hat{\sigma}=\sqrt{\frac{1}{S-1} \sum_{i=1}^{S}\left(\hat{v}_{i}-\bar{v}\right)^{2}} .
\end{gathered}
$$

We set out two factors that will be used to evaluate the quality of the blood velocity estimation methods. The errors that may occur in statistical data evaluation may be divided into two classes. The first is a standard error (or random error) that will always happen since individual estimates of the velocity are subject to variation from one realization to another. The random error of an estimator is measured by the standard deviation estimated by (3.3.7).

The second type of error is a systematic error that will appear with the same magnitude from one estimate to the next, and is called bias error. When there is no bias the estimates are sometimes larger and sometimes smaller than the true value, but on average they are just right. The bias error is measured by the difference between the sample mean (3.3.6) and the true (imposed) value $v_{m}$ being estimated, that is:

$$
\hat{b}=\frac{1}{S} \sum_{i=1}^{S} \hat{v}_{i}-v_{m} .
$$


It is desirable that the averaged value of the estimates is equal to the parameter being: estimated, so that the bias is zero. Furthermore, it is desirable that the estimates are highly concentrated around the true value, so that the standard deviation is as small as possible. These statistics (3.3.8) and (3.3.7) serve as the criteria by which we will judge the quality of an estimator.

\subsubsection{Cramér-Rao Inequality and Efficiency}

In the previous section two quality measures of an estimator, bias and standard deviation, were considered. Another principal factor that can be used to establish the quality of an estimator is the Cramér-Rao lower bound.

Consider the situation where $\theta$ is a scalar parameter to be estimated from a vector of observations $\mathbf{y}$. The mean-square error value (variance) is defined as:

$$
\sigma^{2}=\mathcal{E}\left[\left(\hat{\theta}(\mathbf{y})-\theta_{0}\right)^{2}\right]
$$

where, $\theta_{0}$ denotes the true value of $\theta$, and expected value operator, $\mathcal{E}[\cdot]$, defined by (3.3.1) is evaluated for the probability density function for $\mathbf{y}$, given $\theta_{0}$.

We may be interested in selecting estimators that make variance $\sigma^{2}$, as defined by (3.3.9), small. It is then interesting to note that there is a lower limit to the values of $\sigma^{2}$ that can be obtained with various unbiased estimators. This is the so called Cramér-Rao inequality. It is shown in [43] that under some conditions

$$
\mathcal{E}\left[\left(\hat{\theta}(\mathbf{y})-\theta_{0}\right)^{2}\right] \geq \mathcal{F}\left(\theta_{0}\right)^{-1},
$$

where $\mathcal{F}\left(\theta_{0}\right)$ is Fisher's information. If $p\left(y / \theta_{0}\right)$ is the probability density function for $\mathbf{y}$, given $\theta_{0}$, the Fisher's information equals

$$
\mathcal{F}\left(\theta_{0}\right)=\mathcal{E}\left[\frac{\partial^{2} \ln p\left(\mathbf{y} \mid \theta_{0}\right)}{\partial \theta_{0}^{2}}\right] .
$$

From inequality (3.3.10), some authors [50], [18] define $\hat{\theta}(\mathbf{y})$ to be an efficient estimator of $\theta$ if

$$
\mathcal{E}\left[\left(\hat{\theta}(\mathbf{y})-\theta_{0}\right)^{2}\right]=\mathcal{F}\left(\theta_{0}\right)^{-1},
$$

that is, when the lower bound in $(3.3 .10)$ is attained. However, in many cases the minimum attainable variance is larger than the lower bound (3.3.10). Therefore, we link efficiency with variance as suggested by Rao in [55]. An unbiased estimator of a parameter is defined to be efficient if it has the least possible variance and not necessarily a particular value which may not be attainable for any estimator in a given situation. Specifically, according to Bendat [4], if the variance of an estimator is smaller than the variance of another possible estimator, the estimator is said to be more efficient than other possible estimators. That is,

$$
\mathcal{E}\left[\left(\hat{\theta}_{1}(\mathbf{y})-\theta_{0}\right)^{2}\right] \leq \mathcal{E}\left[\left(\hat{\theta}_{i}(\mathbf{y})-\theta_{0}\right)^{2}\right]
$$


where $\hat{\theta}_{1}(\mathbf{y})$ is the estimator of interest and $\hat{\theta}_{i}(\mathbf{y})$ is any other possible estimator. It implies that the values of $\hat{\theta}_{1}(y)$ (over different samples) are more highly concentrated around the true value $\theta_{0}$ than are the values of other possible estimators.

Regardless of whether the lower bound can be achieved or not, its importance is in providing a benchmark for evaluating the performance of an estimator since it describes the best possible properties one can hope for. We will further continue in Chapter 7 by evaluating the expression for the Cramer-Rao bound for the blood flow velocity estimators.

\subsection{Complex Envelope Transformation}

Although, of course, the received RF signal is real, it is nevertheless converient sometimes to regard it as complex valued. Such signal transformation is one of the stages of signal processing for maximum likelihood velocity estimation method. Therefore, in this section we show the relationship between the real signal $x(t)$ and its complex counterparts (pre-envelope, and complex envelope).

The complex envelope $\tilde{x}(t)$ of $x(t)$ with respect to the frequency $f_{c} \in \mathbf{R}_{+}$is

$$
\tilde{x}(t)=\left(x(t)+j x_{h}(t)\right) \exp \left(-j 2 \pi f_{c} t\right), \quad t \in \mathbf{R},
$$

where the real signal $x_{h}(t)$ is the Hilbert transform of $x(t)$. A definition of the Hilbert transform can be found elsewhere, e. g. [42]. It is a $90^{\circ}$ phase shifter, independent of frequency. Given the complex envelope $\tilde{x}(t)$ of a real signal $x(t)$ with respect to a given frequency $f_{c}$, the signal $x(t)$ itself may successively be retrieved as

$$
x(t)=\operatorname{Re}\left[\tilde{x}(t) \exp \left(j 2 \pi f_{c} t\right], \quad t \in \mathbf{R} .\right.
$$

This shows that determining the complex envelope of a real continuous-time signal is an invertible operation, called the complex envelope transformation.

Another complex signal is the pre-envelope $\breve{x}(t)$, also called the analytical signal corresponding to $x(t)$. The pre-envelope $\breve{x}(t)$ of a real continuous-time signal $x(t)$ is

$$
\breve{x}(t)=x(t)+j x_{h}(t) .
$$

It can be shown ([42]) that when the signal $x(t)$ is band-limited, with quality factor $Q>1$, and $f_{c}$ is a frequency within the positive frequency band of $x(t)$, the complex envelope $\tilde{x}(t)$ contains low frequencies only (i. e. it is a low frequency signal). On the other hand, the frequency content of pre-envelope $\breve{x}(t)$ is centered around $f_{c}$ in the positive frequency semiplane, whereas the spectrum of original signal $x(t)$ is bandpass and symmetric with respect to the origin of the frequency plane. Thus in summary, the complex envelope transformation is used to shift the frequency content of the RF signal to lower frequencies while the analytic version of the RF signal is used to remove the symmetry with respect to the origin of the frequency plane. It is 
actually the high frequency, complex valued signal which makes it possible to identify the center of mass of the spectrum in the positive (or negative) semiplane.

Representing the complex envelope in polar and Cartesian forms, respectively, leads to useful interpretations. Let $\tilde{x}(t)$ be the complex envelope of the real continuoustime signal $x(t)$ with respect to the frequency $f_{c}$. Then the time signals

$$
\alpha_{x}(t)=|\tilde{x}(t)|, \quad \phi_{x}(t)=\arg (\tilde{x}(t)),
$$

are called the envelope and phase, respectively, of $x(t)$ with respect to $f_{c}$, and one may write

$$
x(t)=\alpha_{x}(t) \cos \left(2 \pi f_{c} t+\phi_{x}(t)\right), \quad t \in \mathbf{R} .
$$

Function $\arg (\cdot)$ of (3.4.4) returns the phase angles and can be implemented in several ways listed here

$$
\begin{aligned}
\arg (\tilde{x}(t)) & =\arctan \left(\frac{\operatorname{Im}[\tilde{x}(t)]}{\operatorname{Re}[\tilde{x}(t)]},\right. \\
\arg (\tilde{x}(t)) & =\arctan 2(\operatorname{Im}[\tilde{x}(t)], \operatorname{Re}[\tilde{x}(t)]), \\
\arg (\tilde{x}(t)) & =\operatorname{Im}\left[\log \left(\frac{\operatorname{Im}[\tilde{x}(t)]}{\operatorname{Re}[\tilde{x}(t)]}\right)\right] .
\end{aligned}
$$

The simplest expression involves the inverse tangent, a more sophisticated way uses four quadrant inverse tangent, that returns angles between $-\pi$ and $\pi$. Another possibility, comparable to the previous one, obtains the phase angles from a complex logarithm function.

The signals

$$
x_{c}(t)=\operatorname{Re}[\tilde{x}(t)], \quad x_{s}(t)=\operatorname{Im}[\tilde{x}(t)]
$$

are called the in-phase and quadrature components, respectively, of $x(t)$ with respect to $f_{c}$, and

$$
x(t)=x_{c}(t) \cos \left(2 \pi f_{c} t\right)-x_{s}(t) \sin \left(2 \pi f_{c} t\right), \quad t \in \mathbf{R} .
$$

The envelope, phase, in-phase, and quadrature components are related as

$$
\begin{aligned}
\alpha_{x}(t) & =\sqrt{x_{c}^{2}(t)+x_{s}^{2}(t)}, \quad \phi_{x}(t)=\arg \left(x_{c}(t)+j x_{s}(t)\right), \\
x_{c}(t) & =\alpha_{x}(t) \cos \left(\phi_{x}(t)\right), \quad x_{s}(t)=\alpha_{x}(t) \sin \left(\phi_{s}(t)\right) .
\end{aligned}
$$

It is shown in Van Trees [62] that the scheme used for extracting the in-phase and quadrature components employs two ideal low-pass filters provided that the complex envelope of $x(t)$ with respect to $f_{c}$ is low-pass with quality factor $\mathrm{Q}>1$. The signal $x(t)$ is first multiplied by $\exp \left(-j 2 \pi f_{c} t\right)$, then the result is low-pass filtered (averaged) to obtain the in-phase and quadrature components $x_{c}(t)$, and $x_{s}(t)$. This procedure is the same as what is in communication theory known as demodulation (it consists of retrieving the message signal), however when it is used for signals with limited time extent the term phase detection is more appropriate. 


\subsection{Main Processing Concepts for Blood Velocity Estima- tion}

Existing techniques for blood velocity estimation are traditionally classified to the frequency and time domain processing methods [28], [36]. This may suggest that the domain on which the estimators operate is frequency, respective time domain. However, it is not always true. There exist methods that are based on change in the phase at the carrier frequency (commonly considered as the frequency-domain methods), but the processing is actually performed in the time domain. Therefore, we think that the corresponding terminology "frequency/time domain processing" is misleading. We propose a classification scheme based on the type of input data, e.g. low frequency or high frequency signals. The common feature of the methods from the proposed classes is explained next. Let us assume that the transducer transmits pulses of the form

$$
s(x)=\operatorname{Re}\left[\tilde{s}(x) \exp \left(j 2 \pi \xi_{\mathrm{e}} x\right)\right],
$$

where $\xi_{\mathrm{e}}$ is an emitted (central) frequency and $\tilde{s}(x)$ is the pulse's complex envelope. Then the backscattered RF signal due to the $k$-th emission can be written as

$$
e(x, k)=\operatorname{Re}\left[\tilde{e}(x, k) \exp \left(j 2 \pi \xi_{\mathrm{rf}} x\right)\right], \quad k=1, \ldots, M,
$$

where spatial frequency $\xi_{\mathrm{rf}}$ is a (spatial) carrier frequency of the received RF signal. Thus $\xi_{\mathrm{e}}$ has been changed due to the frequency dependent attenuation in depth to frequency $\xi_{\mathrm{r} f}$. One should realize that actually a real valued radio frequency (RF) signal is recorded. A complex signal is obtained by a transformation described in a previous section. Thus in the case of processing low frequency signals, the RF signals are transformed to low frequency with respect to a reference frequency. For the transformation an assumption about the reference frequency - the spatial center frequency $\xi_{\mathrm{rf}}$ is needed. On the other hand, processing of high frequency signals does not involve a signal transformation with respect to a reference frequency, thereby circumventing the problem associated with an assumption about the carrier frequency.

In the following subsections an overview is given of existing methods for processing low frequency signals (Section 3.5.1), and for processing high frequency signals (Section 3.5.2). Their fundamental starting points will be indicated.

\subsubsection{Processing of Low Frequency Signal}

First, in this section we explain in more depth the problem of choosing a reference frequency for the signal transformation applied to shift the frequency content of the RF signal to lower frequencies. Then we review the basic principles of methods from the class of methods that operate on low frequency data.

In Section 2.4 the Doppler equations (2.4.8), (2.4.9), (2.4.12) were introduced from different points of view. The general formulation that includes all special cases was 
given by (2.4.8). The problem is what frequency of the RF signal (carrier frequency) to use in this expression. If a wideband signal is emitted (short pulse), the effect of frequency dependent attenuation should be taken into account since it modifies the central carrier frequency $\xi_{\mathrm{rf}}$ as a function of depth. To avoid this problem, conventional pulsed Doppler systems employ long emission pulses, consisting of up to 10 periods, corresponding to narrowband RF signals. Therefore, it may be assumed that the center frequency of the received RF signal $\xi_{\mathrm{rf}}$ is not changed significantly due to frequency dependent attenuation effects and is equal to the emitted frequency $\xi \mathrm{e}$.

In concrete terms, for a narrowband process, the signal at depth $x_{1}$ is the local convolution of the 3D-scatterer distribution with the emitted pulse (sample volume). After correction of the phase of the received signal for the time delay due to the change in depth, conventional Doppler systems average the received signal over a depth range to improve the signal-to-noise ratio. This is equivalent to multiplying the received signal by $\exp \left(-j 2 \pi \xi_{\mathrm{e}} x\right)$, and averaging over the depth range of interest, which was mentioned in the previous section as phase detection. The effect of this operation is a conversion of the RF return to a sample of the complex envelope of the pulsed Doppler signal at depth $x_{1}$ as shown in Fig. 2.2 at the intersection. Taking this operation over several RF returns results in the complex envelope of the Doppler signal at depth $x_{1}$, sampled with pulse repetition frequency $f_{\text {prf }}$ (see. Fig. 2.2, signal at right side). The procedure (the mixing and averaging stage) is in fact the complex envelope transformation (Section 3.4) that translates the spectral content of the Doppler signal by $\xi_{\mathrm{e}}$ towards zero frequency.

The goal in conventional pulsed Doppler systems is then to estimate the mean phase shift between successive (transformed) signals. Since instantaneous frequency is time derivative of a phase shift, the frequency shift can be obtained. The estimate of velocity then follows from Doppler equation (2.4.12). Various techniques associated with the above concept are reported in [2], [3], [9]. The correlation concept for estimation of the phase shift can be found in Kasai et al. [38] and Kristoffersen [41]. It was shown that the argument (phase angle) of the complex auto-correlation function of Doppler signal at depth $x_{1}$ at unit sample lag is a close approximation to the mean phase shift (mean frequency shift).

For optimal performance in conventional Doppler, the depth range of interest should match the duration of the emitted pulse [31], [41]. The longer observation range hardly affects the axial resolution because of the length of the pulse employed. Needless to say, the averaging procedure over depth, to improve the Doppler signal, may also be executed at another stage of signal processing. A subsample volume processing as was investigated by Hoeks et al. [30] may be another alternative.

Another concept that employs processing of low frequency signals is based on the maximum likelihood criterion. It was developed for Doppler radar in [63] and has been applied to Doppler ultrasound by Ferrara and Algazi [19], [21]. 'The final processing involves correlation of the received signals with an assumed reference signal. An analysis and comparison of this concept follows in Chapters 5 and 7. 


\subsubsection{Processing of High Frequency Signal}

As was explained in Section 2.4.3, the received RF signal is a real valued, high frequency signal. Estimation methods based on the processing of high frequency signals do not involve a signal transformation with respect to a reference frequency, therefore, it is possible to design an estimator capable of estimating the Doppler (temporal) as well as the carrier (spatial) frequency. Then, for example by obtaining the explicit estimates of both mean Doppler and spatial frequency, the evaluation of the Doppler equation (2.4.8) will provide an estimate of mean velocity. Such an rstimator was investigated by Loupas et al. [46], [45] employing the auto-correlation approach of Kasai et al. [38] to extract both frequencies from the analytic version of the backscattered signal (pre-envelope defined by equation 3.4.3).

Other investigators have suggested measuring the blood velocity from the mean displacement obtained from the cross-correlation function (Bonnefous et al. [8], Foster et al. [23]). It is based on a principle that looking at correlation it may be determined for what depth shift the received RF signals are best aligned. The position of the peak of the cross-correlation function reveals the mean distance covered per time interval $\left(T_{\text {prf }}\right)$ which can be readily converted to the mean velocity.

To be more explicit, let us consider $\mathrm{RF}$ returns due to the pulse $k-1$ and $k$, $\epsilon(x, k-1), e(x, k)$, respectively. From our analysis in Section 2.4 we know that there exist similarity over subsequent signals. This similarity can be described by correlation. 'The cross-correlation, $\gamma(\Delta x)$, of two successive signals is only a function of the depth shift $\Delta x$ due to temporal stationarity of the RF signal (Section 2.4.1). According to (3.3.3), $\gamma(\Delta x)$ is defined as

$$
\gamma(\Delta x)=\int_{-\infty}^{\infty} e(x, k-1) e(x+\Delta x, k) d x .
$$

Applying equation (2.4.1) into the above correlation formula yields

$$
\gamma(\Delta x)=\int_{-\infty}^{\infty} e(x, k-1) e\left(x+x_{r}+\Delta x, k-1\right) d x .
$$

Using expression (2.4.2) for $x_{\tau}$, we can write

$$
\gamma(\Delta x)=\gamma_{0}\left(x_{\tau}+\Delta x\right)=\gamma_{0}\left(v \cos \theta T_{\mathrm{prf}}+\Delta x\right),
$$

where $\gamma_{0}(x)$ is an auto-correlation function. Since $\gamma_{0}\left(x_{r}+\Delta x\right)$ is maximal when its argument is zero, the position of the peak of the cross-correlation function provides the measurement of a mean distance, $x_{\tau}$, travelled by the scatterers moving at velocity $v \cos (\theta)$ in the direction of a beam within the pulse repetition interval $T_{\text {prf }}$.

For a sampled system, the integral of (3.5.3) and (3.5.4) is replaced by a discrete sum. The aim is to locate the peak of the cross-correlation function. The precision depends very much on the depth sampling frequency. The higher the sampling frequency, the better the peak of the cross-correlation can be identified. Performing cross-correlation of the signals sampled at high frequency however, demands higher computational efforts. Some modifications, like interpolation of the function in the 
vicinity of a suspected peak [12], or temporal and spatial clustering techniques [35], [34] were introduced to improve the probability of correct peak detection. Residue Number System (RNS) implementation was proposed in [26], [27] to perform highspeed addition and multiplication for the correlation. These modifications reduce computational effort but require more sophisticated hardware.

To improve the speed of processing and to decrease the hardware requirements the cross-correlation model (CCM) method was developed for estimation of blood velocities [29]. The derivation is based on the model of the cross-correlation function and yields the explicit expression for velocity estimate. In such a way the peak searching procedure is avoided. In the previous study this concept was developed for narrowband signals. The extension of the CCM principle to the wideband signals, already reported in [66], is the subject of Chapter 6 . 


\section{Chapter 4}

\section{A Simulation Model of the Backscattered Signal from Blood}

\subsection{Introduction}

In this chapter we concentrate on the simulation model of the signal, backscattered from blood, whose physical properties were described in Chapter 2. The model should be suitable for computer simulation, since it will be applied at the data generation stage in our analysis of estimation methods in the following chapters. We will typically run two different algorithms on the same set of simulated backscattered $\mathrm{RF}$ signal to investigate various aspects of different processing methods for velocity estimation. A qualitative assessment of the performance of estimators is be possible when the underlying properties of the backscattering process which the generated signals aim to model are completely known.

The existing models in literature can be classified according to Mo et al. [49] into three categories:

- Models based on the physics of scattering as studied by Angelsen [1]. Physical modeling of the pulse-echo loop is quite complex, even if simplifying assumptions are made about the transducer excitation, the properties of tissue, and characteristics of the scattering interactions.

- Models based on the stochastic nature of the signal which implies that the backscattered signal can be considered as a form of bandlimited Gaussian noise (Mo \& Cobbold [48], Jones \& Giddens [37], Hoeks et al. [29]).

- Parametric spectral models as autoregressive (AR), moving average (MA), autoregressive moving average (ARMA) models. Fitting of an assumed model from this category to the Doppler signal is problematic, because it is not clear how the order of different parametric models and their parameters are related to the physical processes that give rise to the Doppler signal [61].

The second category mentioned in the begining of this section, looks to be the most straightforward choice for the purpose of this thesis. We will use a signal model proposed by Hoeks [29] that simulates a stationary signal backscattered from a sample 
is that the averaged backscattered RF spectrum coincides with the spectrum of the transmitted pulse, while individual spectral heights fluctuate erratically about their average value. This property stems also from the fact that the backscattered signal originates from a large number of scatterers of random positions as well as scattering strength.

The actual received signal $e(x, k)$ is formed by the signal $r(x, k)$ returned from the scatterers, and a signal $w(x, k)$ which represents the effect of instrumentation noise. Its spectral characteristics are mainly determined by the bandpass character of the transducer and receiver, hence

Assumption 4.7 The spectrum of the additive RF noise is bandlimited with the shape of a Gaussian curve.

By the above assumptions the signal space is specified. The properties are based on the physical nature of the ultrasound echo signal from blood as developed in Section 2.4. All restrictions considered here are realistic, they are common, and often implicit in Doppler ultrasound.

\subsection{Input Parameters for the Model}

In this section we point out the input parameters for the simulation model and give their specifications used in generation of simulated signals for an analysis of the estimation methods reported in the following chapters.

The simulation model provides data represented in matrix form as shown in figure 3.1. Its dimensions are specified by $M$ rows (number of observations) and $N$ columns (number of samples in depth) which was treated in Section 3.2. The number of observations is limited by the temporal stationarity of the RF signal. From the discussion in Section 2.4.1 it can be concluded that the time-invariant velocity field exists over 50 to 100 subsequent $R F$ returns if the pulse repetition period equals 0.1 ms. In order to have some reserve, $M$ equal to 32 observations will be considered as the maximal time window. The effect of this parameter will be investigated for $M$ $=4,8,16,25$, and 32 observations.

The sampled backscattered signal should be generated with a sampling ratio in depth $R_{\mathrm{S}}$ of at least 4 as follows from the Nyquist bound described by (3.2.1). Our choice is confined to this value in order to process as few samples as possible (low computational effort). An oversampling factor $R_{\text {over }}$ can be entered to resample and decimate the signal for more accurate simulation of phase change due to the movement in Section 4.4.2. In the previous application in [29] it was shown that oversampling by a factor 4 is sufficient, therefore in our simulation experiment $R_{\text {over }}$ is set to 4 .

The number of columns $N$ of a data matrix is given by the size of the window in depth, $N_{\mathrm{p}}$ periods, multiplied by $R_{\text {over, }}$, and so $N=N_{\mathrm{p}} R_{\text {over. }}$. For optimal 
performance in terms of signal-to-noise ratio and axial resolution, the length of depth window should match the duration of emission [40]. In Section 2.3.1 it was shown that the quality factor, $Q_{\mathrm{rf}}$, equals the duration of the impulse response, therefore in our simulations we consider the size of depth window for $N_{\mathrm{p}}=k Q_{\mathrm{rf}}$ with $k \in \mathbf{Z}$.

The velocity field is defined by the scaled mean velocity $\vartheta_{\mathrm{m}}$, and scaled velocity bandwidth (spread) $\vartheta_{\mathrm{s}}$. Within that bandwidth, $N_{\mathrm{v}}$ velocity components are evenly distributed between $\vartheta_{\mathrm{m}}-\vartheta_{\mathrm{s}} / 2$, and $\vartheta_{\mathrm{m}}+\vartheta_{\mathrm{s}} / 2$. For simulations $N_{\mathrm{v}}$ is selected to be 21 in accordance with the choice in [29]. The scaled velocities $\vartheta$ are expressed in units of $\lambda / T_{\mathrm{prf}}$, in order to have it as a fraction of the carrier frequency $\xi_{\mathrm{rf}}$. The conversion formula to velocity $v$ expressed in $\mathrm{ms}^{-1}$ is therefore

$$
v=\frac{1}{2} \vartheta \frac{f_{\mathrm{prf}}}{\xi_{\mathrm{rf}}}=\frac{1}{2} \vartheta \frac{c}{f_{\mathrm{rf}}} f_{\mathrm{prf}}=\frac{1}{2} \vartheta \frac{\lambda}{T_{\mathrm{prf}}},
$$

where (2.4.4) was substituted for $\xi_{\mathrm{rf}}$, and the wavelength $\lambda=c / f_{\mathrm{rf}}$. Factor $1 / 2$ is meant to incorporate the round trip. The scaled velocity range is limited to $-0.5 \leq v \leq 0.5 \lambda / T_{\text {prf }}$. The reason for this is that aliasing (Section 2.4.4) occurs if the change in pathlength is greater than half the wavelength $\lambda$. Furthermore, it spans positive as well as negative velocities. The sign indicates the direction of flow (towards or away from the transducer).

The relationship between the dimensionless velocity $\varphi$, and the scaled velocity $\vartheta$ is derived by comparison of right-hand sides of equations (4.3.1) and (3.2.4) (where $\cos \theta=1$ by Assumption 4.4). The result is

$$
\vartheta=\varphi \frac{\xi_{\mathrm{rf}}}{\xi_{\mathrm{s}}}=\frac{\varphi}{R_{\mathrm{s}}}
$$

where $R_{\mathrm{S}}$ is a sampling ratio in depth.

The next input parameter is the quality factor for a signal $Q_{\mathrm{rf}}$ as defined by (2.4.13). It characterizes the spectral properties of the signal as was explained in Section 2.3.1. Because of Assumption 4.5 and a discussion in Section 2.4.3, it is related to the quality factor of the transducer as $Q_{\mathrm{rf}} \geq Q_{\mathrm{tr}}$. Since we are interested more in the processing of the wideband signals, the quality factor will vary between 2 and 8 . The spectral characteristics of the noise signal are given by quality factor $Q_{\mathrm{n}}$ which always satisfies $Q_{\mathrm{n}} \leq Q_{\mathrm{rf}}$. In our setting, $Q_{\mathrm{n}}=Q_{\mathrm{rf}}$ which is in accordance with the expected relation.

The portion of noise in the backscattered signal is given by the signal to noise ratio, SNR stated in $\mathrm{dB}$ (2.4.19). In many cases the SNR will be considered at $10 \mathrm{~dB}$, since this value describes a typical environment of the blood velocity measurements.

To summarize, all necessary input parameters are listed here:

$$
\text { MODEL parameters }=\left(M, N_{\mathrm{p}}, R_{\mathrm{s}}, R_{\text {over }}, \vartheta_{\mathrm{m}}, \vartheta_{\mathrm{s}}, N_{\mathrm{v}}, Q_{\mathrm{rf}}, Q_{\mathrm{n}}, \mathrm{SNR}\right),
$$

where several parameters are maintained at standard values through this study, producing the type of signal commonly found in practice. As already explained above, in our study we keep $R_{\varsigma}=4, R_{\text {over }}=4, N_{\mathrm{v}}=21, Q_{\mathrm{n}}=Q_{\mathrm{rf}}$ unless otherwise stated. 


\subsection{Modeling of the Backscattered Signal from Blood}

This section describes a scheme for the simulation of a two-dimensional set of received echoes (depth samples versus pulse transmissions) having the characteristics given by Assumptions 4.1- 4.6. This simulation procedure was introduced in [29] and has already been applied to other similar studies [64], [31], [30]. Here, it is presented in a slightly different fashion and studied in detail, based on the outlined properties of the signal in Sections 4.2 and 2.4 .

To recapitulate, consider the two-dimensional Fourier transform of the received signal with respect to spatial and temporal frequency. According to expression (2.4.5) the frequency content of the signal will be concentrated around a straight line in the frequency plane. This line will pass through the origin and have a slope linearly proportional to the velocity of the scatterers. A return due to a single pulse, $e(x, k)$, has by Assumption 4.6 an expected power spectrum (as a function of spatial frequency) equal to the power spectrum of the transmitted pulse. Furthermore, as a consequence of Assumption 4.3, the phase of this returned signal is random since $e(x, k)$ is composed of signals backscattered by a large number of randomly located scatterers.

The main idea of the simulation model is based on the fact that the transducer acts as a bandpass filter. After activation, the transducer generates its impulse response. According to Assumption 4.1 the frequency response of the transducer has a Gaussian shape as shown in figure 4.1. The signal is reflected by randomly distributed scatterers of different scattering strength, imposing a random phase as well as an amplitude variation on the summated signal received by the transducer. There it is again bandpass filtered in the conversion process from acoustic to electric signal. In pulsed wave systems ultrasound pulses are transmitted with pulse repetition frequency yielding a collection of RF returns as illustrated in figure 2.2. Due to the changing distance between transducer and groups of moving scatterers gradual translation is manifested between successive returns. We repeat again that the received signal due to one pulse does not contain any information about the velocity of the scatterers.

The simulation procedure starts with the construction of a long waveform as described in Section 4.4.1 by bandpass filtering of noise sequence, where the filter has the Gaussian shape. The subsequent signals are extracted from this long waveform in such a way that they are shifted versions of each other. 'The size of the shift depends on the imposed velocity of moving scatterers. This will be described in Section 4.4.2. At the end of the simulation procedure the noise signal is added to each observation according to Assumption 4.7 and a given signal-to-noise ratio.

The next three Sections 4.4.1, 4.4.2, 4.4.3 describe the process of obtaining the sequence of RF returns in a matrix structure $\mathbf{D}(M, N)$ as illustrated in figure 3.1. For simulation of more sets of matrices the same procedure has to be repeated. 

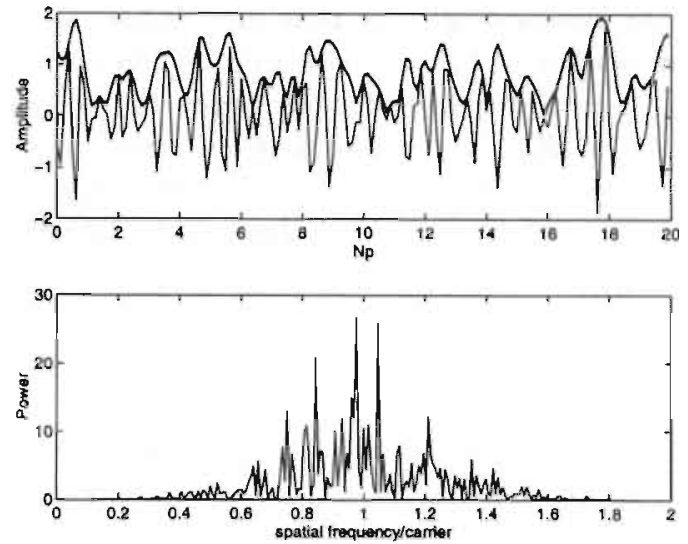

Figure 4.2. An example of a generated waveform over 20 periods $\left(N_{\mathrm{p}}\right)$, the envelope is plotted in bold line (top). The corresponding power spectral distribution is shown at the bottom. It is obtained with $Q_{\mathrm{S}}=2, R_{\mathrm{S}}=4, R_{\text {over }}=1$, and based on 512 frequency bins.

\subsubsection{Generation of the Waveforms}

For each component within the (scaled) velocity bandwidth a waveform $w^{j}\left(n_{w}\right)$, where $j=1, \ldots, N_{\mathrm{v}}$ is to be generated. Thus at the first stage a number of $N_{\mathrm{v}}$ waveforms are independently formed according to the following steps:

1. Complex arrays $X^{j}(\Psi)$ are composed of real and imaginary parts that have independent zero mean Gaussian distributed values. The data sequences are supposed to be long enough, a length of series $L_{\text {serie }} \geq R_{\mathrm{s}} N_{\mathrm{p}(\mathrm{rf})} M+\left(\vartheta_{\mathrm{m}}+\vartheta_{\mathrm{s}}\right) R_{\mathrm{s}} M$. The $L_{\text {serie }}$ defines a number of discrete frequencies and is usually chosen to be a power of two in connection with the Fast Fourier Transform algorithm. $X^{j}(\Psi)$ is a function of normalized spatial frequency $\Psi=\left(0, \ldots, L_{\text {serie }}\right) / L_{\text {serie }}$, defined as $\Psi=\xi / \xi_{s}$, while $\xi_{s}$ is the sampling frequency specified in Section 3.2. Notice, that because of repetition of the spectra at intervals of sampling frequency, the frequency content at interval $\Psi=1 / 2, \ldots, 1$ is the same as the frequency content at interval $\Psi=-1 / 2, \ldots, 0$.

2. Each array $X^{j}(\Psi)$ is multiplied by a Gaussian shaped function that determines the bandpass filter. The function follows from Assumption 4.1 and is defined by

$$
G(\Psi)=\sqrt{|S(\Psi)|^{2}} \quad \text { for } \Psi=0, \ldots, 1 / 2,
$$

where $|S(\Psi)|^{2}$ is given by equation (4.2.1) centered on $\Psi_{\mathrm{rf}}=1 /\left(L_{\text {serie }} R_{8}\right)$. The resulting data $W^{j}(\Psi)=X^{j}(\Psi) G(\Psi)$ represents a bandpass filtered signal with random, uniformly distributed phase and the magnitude variations of a Rayleigh distribution. 
3. To prevent the resampling error that may occur by the interpolation scheme in step 4 of Section 4.4 .2 an oversampling factor $R_{\text {over }}$ is introduced. It causes an intermediate sampling frequency to increase to $\xi_{\text {over }}=R_{\text {over }} \xi_{\mathrm{s}}$. So in the case of $R_{\text {over }}>1$, the previous two steps should be realized for normalized frequency $\Psi=\xi / \xi_{\text {over }}$. To incorporate this, the series $W^{j}(\Psi)$ a.re then padded with zeros to the appropriate length $L_{\text {over }}=R_{\text {over }} L_{\text {serie }}$ for $\Psi=\left(0 \ldots, L_{\text {serie }}, \ldots, L_{\text {over }}\right) / L_{\text {over }}$. The function (4.4.1) is then centered at $\Psi_{\text {rf }}=1 /\left(L_{\text {serie }} R_{\mathrm{S}} R_{\text {over }}\right)$.

4. Using the inverse fast Fourier transform algorithm, $W^{j}(\Psi)$ is converted into the waveform $w^{j}\left(n_{w}\right)$, for $n_{w}=1, \ldots, L_{\text {over }}$ and the mean power of $w^{j}\left(n_{w}\right)$ is set to one. The real part of the signal is taken for analysis in order to have a signal compatible with practice.

An illustrative example of an individually generated waveform $w^{1}\left(n_{w}\right)$ gives figure 4.2. The waveform is plotted together with its envelope at the top. The bottom part shows the corresponding power spectral distribution with quality factor $Q_{\mathrm{s}}=2$. This individual distribution obeys approximately the power spectrum shown at Fig. 4.1 , except for the variations in the expected shape.

\subsubsection{Generation of Successive Signals}

The next stage is to compose a sequence of RF signals for $k=1, \ldots, M$ with temporal relationship that models the mean phase shift between successive signals according to the desired mean velocity. The idea is as follows: $N_{\mathrm{v}}$ sequences of partial signals $r^{i}(n, k)$ are arranged so that each sequence models the gradual translation that simulates one desired velocity component within the velocity bandwidth. The final sequence of RF signals $r(n, k)$ is compounded as a summation of $N_{\mathrm{v}}$ partial signals for $k=1, \ldots, M$.

In concrete terms, a sequence of $M$ backscattered RF signals is formed from the waveforms $w^{j}(n)$ for $j=1, \ldots, N_{\mathrm{v}}$, in the following way:

1. A rectangular observation window of the size specified by a number of periods $N_{\mathrm{p}}$ at the carrier frequency is applied on each waveform $w^{j}\left(n_{w}\right)$. The number of sample points within the observation window $N$ from the data matrix of Fig. 3.1 (in depth) then equals to $N_{\mathrm{p}} R_{\mathrm{s}} R_{\text {over }}$. From now on, the range of a variable $n$ is $1, \ldots, N$.

2. An initial position of the window $z^{j}$ for the first return $(k=1)$ is chosen in the middle of the waveform at position $L_{\mathrm{over}} / 2$, for all $j=1, \ldots, N_{\mathrm{v}}$.

3. The temporal relationship between consecutive $\mathrm{RF}$ returns follows from the imposed scaled mean velocity $\vartheta_{\mathrm{m}}$, and imposed scaled velocity spread $\vartheta_{\mathrm{s}}$, and 
is given by shift $d^{j}(k)$ for the $j$-th serie at time $k$ as:

$$
d^{j}(k)=(k-1) \frac{1}{\Psi_{\mathrm{rf}}}\left(\vartheta_{\mathrm{m}}+\vartheta_{\mathrm{s}}\left(\frac{2 j-N_{\mathrm{v}}-1}{2\left(N_{\mathrm{v}}-1\right)}+\frac{a^{j}(k)}{N_{\mathrm{v}}}\right)\right), j=1, \ldots, N_{\mathrm{v}}
$$

where $\Psi_{\mathrm{rf}}$ is a carrier (normalized) frequency of the RF signal which is by Assumptions $4.1,4.5$ and 4.6 equal to the normalized emitted frequency, $\Psi_{\mathrm{e}}$. Suppose, $\vartheta_{\mathrm{s}}=0$, then the ratio $\vartheta_{\mathrm{m}} / \Psi_{\mathrm{rf}}$ gives the fraction of carrier frequency that has been changed per pulse repetition period due to the motion of (scaled) speed $\vartheta_{\mathrm{m}}$. If $\vartheta_{\mathrm{s}} \neq 0$, the first term within the inner brackets next to $\vartheta_{\mathrm{s}}$ ensures that the $N_{\mathrm{v}}$ velocity components are evenly distributed between $\hat{\vartheta}_{\mathrm{m}}-\hat{\vartheta}_{\mathrm{s}} / 2$ (for $j=1$ ) and $\vartheta_{\mathrm{m}}+\vartheta_{\mathrm{s}} / 2$ (for $j=N_{\mathrm{v}}$ ). A number $a^{j}(k)$ in the second term is drawn from a uniform distribution within the interval $(-1 / 2,1 / 2)$ and causes a small random fluctuation around each component.

Thus $d^{j}(k)$ denotes the change in phase per pulse repetition period, or in other words, the fractional number of sampling points the following return should be shifted with respect to the current one. This is not always an integer, therefore interpolation in order to accurately simulate the gradual translation over successive returns may be necessary.

4. For $j=1, \ldots, N_{\mathrm{v}}$ a number of $M$ observations are extracted from waveform $w^{j}\left(n_{w}\right)$. The contribution of the $j$-th waveform $w^{j}\left(n_{w}\right)$ to the $j$-th partial RF signal $r^{j}(n, k)$ at time $k$ is given for all $k=1, \ldots, M$ as

$$
r^{j}(1, k), \ldots, r^{j}(N, k)=w^{j}\left(z^{j}-d^{j}(k)\right), \ldots, w^{j}\left(z^{j}-d^{j}(k)+N\right) .
$$

Non-integer shifts $d^{j}(k)$ are implemented by applying a linear interpolation between sample points.

As $d^{j}(k)$ models the gradual translation of the subsequent returns (due to the changing distance between the transducer and groups of moving scatterers), the sequence of $M$ fractions $r^{j}(n, 1), \ldots, r^{j}(n, M)$ at fixed $j$, where $j=1, \ldots, N_{\mathrm{v}}$, represents a backscattered signal from the environment with a constant mean velocity.

5. The partial signals $r^{j}(n, 1), \ldots, r^{j}(n, M)$ for all $j=1, \ldots, N_{\mathrm{v}}$ are decimated by the factor $R_{\mathrm{over}}$ so that the sample frequency is reduced from $\xi_{\text {over }}$ to $\xi_{\mathrm{s}}$.

6. The backscattered signal $r(n, k)$ at a fixed time $k$ is composed as a summation of $N_{\mathrm{v}}$ partial signals:

$$
r(n, k)=\sum_{j=1}^{N_{\mathrm{v}}} r^{j}(n, k) .
$$

By this, different velocity components are included in the simulated backscattered signal $r_{k}(n, k)$. 


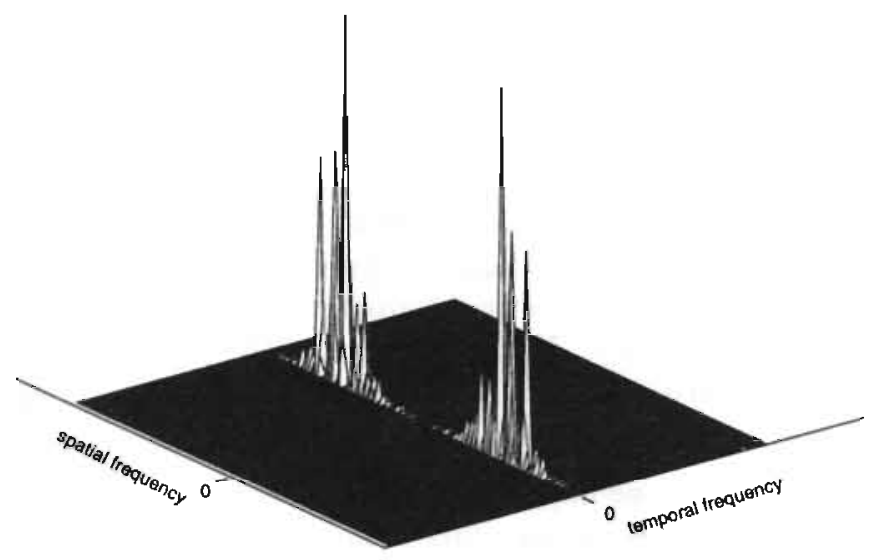

(a)

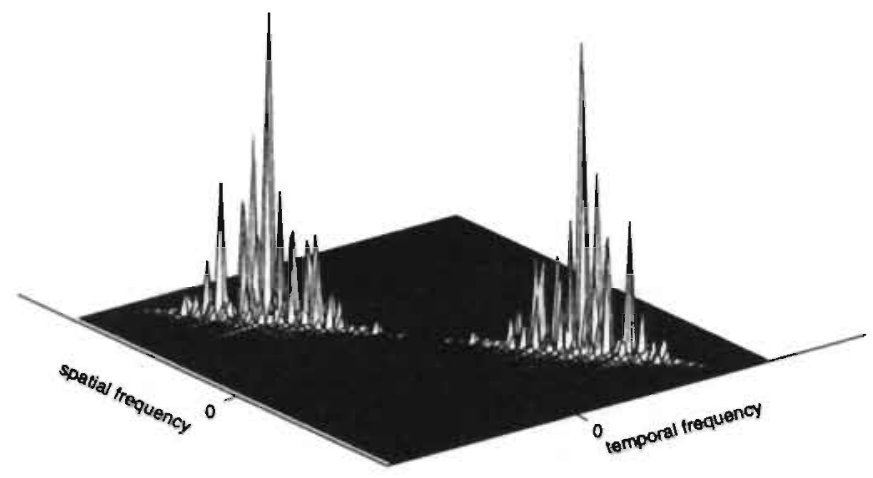

(b)

Figure 4.3. Power spectrum of the generated data with zero (a) and nonzero (b) imposed mean velocity. The model parameters were set at $M=64$ observations, $N_{\mathrm{p}}=32$ periods, $\vartheta_{\mathrm{s}}=0, Q_{\mathrm{rf}}=2, \mathrm{SNR}=40 \mathrm{~dB}, \vartheta_{\mathrm{m}}=0(\mathrm{a})$, and $\vartheta_{\mathrm{m}}=0.2 \lambda / T_{\mathrm{prf}}(\mathrm{b})$. 


\subsubsection{Generation of Signal with the Desired SNR}

To achieve the given signal-to-noise ratio SNR, a noise signal $w(n, k)$ is added to each signal $r(n, k)$ independently. The steps are the following:

1. According to the steps in Section 4.4 .1 a waveform $w\left(n_{w}\right)$ is formed. Its spectral properties are also modelled by (4.4.1), because of Assumption 4.7.

2. From a series $w\left(n_{w}\right)$ segments $w(n, k)$ of length $N$, specified in step 2 . of Section 4.4 .2 , are extracted with random initial position of window placement for all $k=1, \ldots, M$.

3. The noise signal $w(n, k)$ and backscattered signal $r(n, k)$ are mixed according to the imposed signal-to-noise ratio converted from $\mathrm{dB}$ to the dimensionless number $\mathrm{SNR}_{\text {ratio }}(2.4 .19)$. It yields the simulated received signal $e(n, k)$

$$
e(n, k)=\mathrm{SNR}_{\mathrm{ratio}} r(n, k)+w(n, k) .
$$

The above formula gives for $n=1, \ldots, N$ and for $k=1, \ldots, M$ the data matrix structured as in figure 3.1.

\subsection{Example of Simulated Signal}

This section deals with the analysis of the actual two-dimensional spectral characteristics of the RF signal that was generated by the simulation model introduced in the previous sections. According to our earlier discussions in Section 2.4.1, the spectrum should be concentrated around a straight line passing through the origin of the frequency plane. The line should have a slope proportional to the mean velocity. In this section, we will examine whether the example of the generated RF signal obeys this feature.

The parameter setting for the simulation model as defined by (4.3.3) is fixed at the following values: $M=64$ observations, $N_{\mathrm{p}}=32$ periods, $\vartheta_{\mathrm{m}}=0$ or $0.2 \lambda / T_{\text {prf }}$, $\vartheta_{\mathrm{s}}=0$, or $0.1 \lambda / T_{\mathrm{prf}}, Q_{\mathrm{rf}}=2, \mathrm{SNR}=40 \mathrm{~dB}$. Other parameters of (4.3.3) were fixed at constant values specified in Section 4.3 .

The power spectrum of the generated data for zero imposed mean velocity is depicted. in figure 4.3 (a) and for nonzero imposed mean velocity in figure 4.3 (b). We see that the components of the two-dimensional power spectrum consist of two segments on a line passing through the origin in spatial-temporal frequency domain. In Fig. 4.3 (a) the slope of this line is clearly zero which corresponds to the imposed mean velocity $\vartheta_{\mathrm{m}}=0$. The slope of the line in Fig. 4.3 (b) will be examined next.

The contour lines, the equal-height loci, of the power spectrum of Fig. 4.3 (b) are shown in figure $4.4(\mathrm{a})$. Indeed, the spectral components are concentrated on a line with a slope $\varphi=0.8$ as follows from (3.2.3). Dividing this dimensionless number by a sampling ratio $\left(R_{\mathrm{S}}=4\right)$ will yield the value of the mean velocity scaled with 


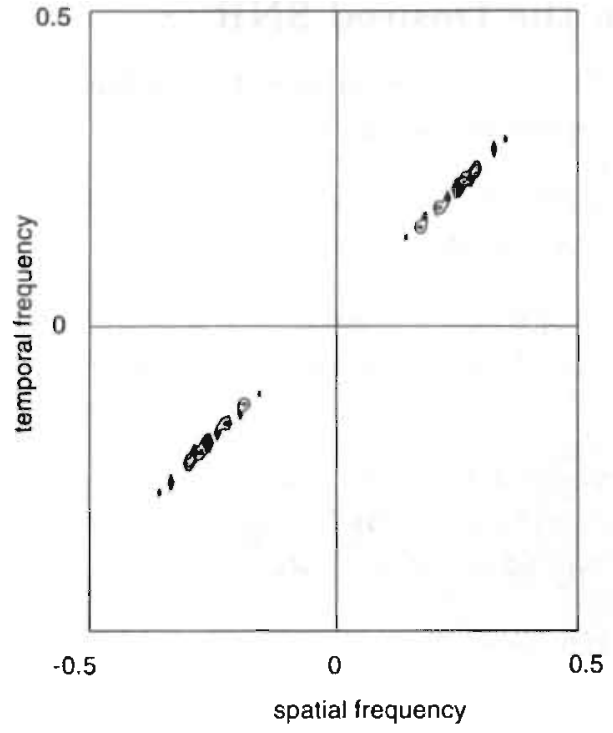

(a)

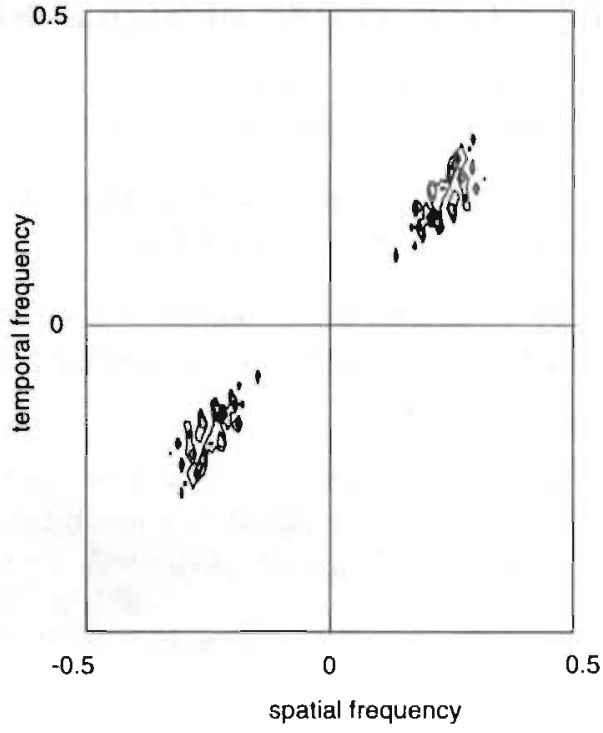

(b)

FigURE 4.4. Contonr plots of the two dimensional power spectrum of the generated sequence of signals for two various scaled velocity bandwidths, $\vartheta_{\mathrm{s}}=0,(\mathrm{a}), \vartheta_{\mathrm{s}}=$ $0.1 \lambda / T_{\mathrm{prf}}$, (b). Other model parameters are fixed at: $M=64$ observations, $N_{\mathrm{p}}=$ 32 periods, $\vartheta_{\mathrm{m}}=0.2 \lambda / T_{\mathrm{prf}}, Q_{\mathrm{rf}}=2, \mathrm{SNR}=40 \mathrm{~dB}$.

respect to $\lambda / T_{\mathrm{pr} f}$ (this follows from 4.3.2). Also in this case the scaled mean velocity $\vartheta=0.2 \lambda / T_{\text {prf }}$ is in accordance with the imposed scaled mean velocity, $\vartheta_{\mathrm{m}}$.

For comparison, the contour plot for the nonzero velocity bandwidth $\left(\vartheta_{\mathrm{s}}=\right.$ $\left.0.1 \lambda / T_{\text {prf }}\right)$ is depicted in figure $4.4(\mathrm{~b})$. Obviously, the slope is unchanged, only the line is more smeared which is a consequence of spectral broadening due to the different velocity components simulated within the sample volume.

Before leaving this section we point out that the purpose of this chapter has been to present the simulation model for the pulsed backscattered signal. We have indeed seen that the proposed simulation procedure yields signals with the desired properties. The resulting sequence of $\mathrm{RF}$ signals in a matrix structure as illustrated in figure 3.1 will be an input for analysis of various processing methods in the following chapters. 


\section{Chapter 5}

\section{The Maximum Likelihood Method}

\subsection{Introduction}

System identification methods are often applied in signal processing in order to extract features of interest. The area of system identification [43], [57] deals with the problem of extracting information from observations that themselves could be unreliable. From the previous chapters we know that the recorded sequence of RF signals we have to deal with contains useful information, because subsequent signals are related. However, in the presence of noise this relationship is not well defined. An assumed relationship among observed signals involves parameters that should be estimated, in our case it is a velocity.

This chapter we shall confine to one of the common parameter estimation methods, to the maximum likelihood (ML) method. It will be applied to the estimation of blood velocity from backscattered ultrasound signals.

The principle of maximum likelihood estimation is usually described within a probabilistic framework. The observations are then thought of as realizations of stochastic variables. There are several ways to describe the concept of the maximum likelihood estimation procedure that we shall use. Suppose that the observations are represented by the random variable $y$, a vector of observations that takes values in $\mathbf{R}^{n}$. Let the joint probability density function (pdf) for the random vector to be observed be given by $p(\mathbf{y}, \theta)$, where $\theta$ is a vector of unknown (but fixed) parameters, which completely describes the pdf. After observing $\mathbf{y}$, we shall want to estimate the value $\theta$. A reasonable estimator of $\theta$ could be to select it so that the observed event becomes "as likely as possible".

Since the realizations take values in $\mathbf{R}^{n}$, the probability that a particular realization (= observation) should take exactly the value $\mathrm{y}^{*}$ is zero. In our case, we can define the likelihood via the conditional probability density $p\left(y^{*} \mid \theta\right)$ (for details see Luesink [47]). This is a deterministic function of $\theta$ once the numerical value $\mathrm{y}^{*}$ is inserted. This function is called the likelihood function. It can be interpreted as giving a measure of the plausibility of the data under different parameters. So, it reflects 
the "likelihood" that the observed event should indeed take place. An estimate of $\theta$ that we seek could simply be selected as

$$
\hat{\theta}_{\mathrm{ML}}(\mathbf{y})=\arg \max _{\theta} p\left(\mathbf{y}^{*} \mid \theta\right)
$$

where the maximization is performed for fixed $\mathrm{y}^{*}$. This function is known as the maximum likelihood estimator. Hence, we choose as an estimate the value of $\theta$ that most likely caused a given value of $\mathbf{y}^{*}$ to occur.

In literature, there are two different treatments of the maximum likelihood estimation of blood velocities. They differ in the form of the likelihood function which is a crucial element in ML estimation. In the treatment presented by Talhami et al. in [58] the Kalman filter was applied in order to estimate the ARMA (autoregressive, moving average) models whose likelihood was to be maximized. The velocity followed from the estimated frequency, the mode of the spectrum of the identified ARMA process. In this case, the relation between a parametric ARMA model and physical processes that give rise to the Doppler signal is lost, which has already been mentioned in Section 4.1 with respect to the parametric spectral models (such as ARMA model). This modelling approach falls under the black-box modelling within the system identification. However, instead of the black-box approach we prefer models that make a use of a priori information. They result in an alternative treatment of maximum likelihood estimation that was formulated and developed by Van Trees for Doppler radar in [62], and [63]. For Doppler ultrasound it was applied by Ferrara and Algazi in [19], and [21]. In this case, the likelihood function is a function of velocity and the maximization is carried out over the velocity parameter only. The likelihood function is derived using the "slowly fluctuating point target" assumption from Doppler radar. The evaluation of the maximum likelihood method for blood velocity estimation in [19], and [21] includes only properties of the likelihood function, such as the height of the subsidiary peak, the width and position of the main peak. The theoretical predictions in [19] show promising results for signals that comply with the point target assumption. In [21] the evaluation of the method has been accomplished with an experimental data modelling closely ideal situation (parabolic flow within a tube of radius of $3.5 \mathrm{~mm}$, with the maximum velocity of approximately $0.2 \lambda / T_{\text {prf }}$ and no additive noise). Again, only properties of the likelihood function were evaluated for this particular situation.

Our contribution is directed to the extensive statistical evaluation of the interdependences of this method on signal and system parameters, thereby considering more realistic (simulated) signals. The performance is investigated in terms of bias and standard deviation which also gives the possibility for comparison with other processing techniques for blood velocity estimation.

The organization of this chapter is as follows. Section 5.2 gives a brief summary of the theoretical support for the maximum likelihood estimation method and describes inputs for the simulation procedure. A sample distribution of the maximum 
likelihood estimates of velocity is examined in Section 5.3. Then in Section 5.4 the empirical analysis of the performance (accuracy) of the proposed estimator is given with respect to the various processing and system parameters.

\subsection{Maximum Likelihood Blood Velocity Estimator}

The goal of this section is to illustrate the theoretical development of the maximum likelihood estimator for blood velocity estimation. We shall do it using a concept developed in [62] by means of a simple example in Section 5.2.1. Then we formulate the likelihood function for our specific case, blood velocity estimation in Section 5.2.2. Once the likelihood function is found the maximum likelihood estimator follows from equation (5.1.1). The realization of the maximum likelihood estimator of blood velocities is the subject of Section 5.2.3.

\subsubsection{Theoretical Derivation}

The theoretical derivation of the maximum likelihood estimation will be developed for the continuous time situation for reasons of clarity, but with suitable modification, it may be applied to a discrete array of data.

We first assume that the transducer transmits a cosine wave continuously at frequency $f_{\mathrm{e}}$ during the time $T$ whose envelope is Gaussian. Thus, the transmitted signal $s(t)$, having finite energy and thus a square integrable function $\left(s(t) \in \mathcal{L}_{2}\right)$, can be compactly described by means of a complex representation as given by equation (3.5.1). Suppose that the received signal from a large number of point scatterers moving at velocity $v$, in absence of noise is approximated as

$$
\tilde{r}(t)=\tilde{b} \tilde{s}(t ; v),
$$

where the multiplier $\tilde{b}$ is a zero-mean complex Gaussian random variable with $E[\tilde{b}]=$ 0 and $E\left[|\tilde{b}|^{2}\right]=2 \sigma_{b}^{2}$. Function $\tilde{s}(t ; v)$ is a deterministic function of time $t$ and velocity $v$ is its parameter. Moreover, $\tilde{s}(t)$ equals zero for $t<0$ and $t>T$ and is chosen to satisfy $\int_{0}^{T}|\tilde{s}(t)|^{2} d t=1$. Further in this section we will proceed on the general level. An explicit form of equation (5.2.1) for backscattered signal will be discussed in Section 5.2.2. The next step is to characterize the additive noise process. We assume that there is an additive Gaussian noise $w(t)$ that has a bandpass spectrum so that. we can represent it as

$$
w(t)=\operatorname{Re}\left[\tilde{w}(t) \exp \left(j 2 \pi f_{e} t\right)\right],
$$

where $\tilde{w}(t)$ is a complex envelope to $w(t)$ with respect to the central frequency $f_{\mathrm{e}}$. Thus, the total received waveform is

$$
\tilde{e}(t)=\tilde{b} \tilde{s}(t ; v)+\tilde{w}(t) .
$$


The purpose is to estimate the unknown parameter, velocity $v$, in presence of noise. Alternately, we may wish to specify $\tilde{e}(t)$ being $\mathcal{L}_{2}$ (square integrable) by a countable set of numbers

$$
\tilde{e}(t)=\sum_{i=1}^{\infty} \tilde{e}_{i} \tilde{\Phi}_{i}^{*}(t)
$$

where the $\tilde{\Phi}_{i}(t) i=1, \ldots, \infty$ is a complete set of orthonormal functions in $\mathcal{L}_{2}$. It holds that $\int_{0}^{T} \tilde{\Phi}_{i}(t) \tilde{\Phi}_{i}^{*}(t) d t=\delta_{i j}$, where $\delta_{i j}$ is the Kronecker delta function, providing

$$
\left\|\tilde{\Phi}_{i}(t)\right\|=\int_{0}^{T}\left|\tilde{\Phi}_{i}(t)\right|^{2} d t=1
$$

The coefficients $\tilde{e}_{i}$ of equation (5.2.4) are defined as

$$
\tilde{e}_{i}=\int_{0}^{T} \tilde{e}(t) \tilde{\Phi}_{i}^{*}(t) d t
$$

and they are statistically independent, zero mean complex Gaussian random variables. This is a property of the Karhunen-Loève expansion which has been applied in this case.

Here, the method is straightforward. If we choose the first orthonormal function $\Phi_{1}(t)$ to be the complex envelope of the modified transmitted signal, $\tilde{s}(t ; v)$, the first coefficient in the decomposition, $\tilde{e}_{1}$, is the complex Gaussian random variable,

$$
\tilde{e}_{1}=\int_{0}^{T} \tilde{e}(t) \tilde{s}^{*}(t ; v) d t
$$

Using equation (5.2.3) in (5.2.7) the first coefficient equals

$$
\tilde{e}_{1}=\tilde{b}+\tilde{w}_{1},
$$

where $\tilde{w}_{1}$ is a zero-mean complex Gaussian random variable $\left(E\left[\left|\tilde{w}_{1}\right|^{2}\right]=N_{0}\right)$. Obviously, the sum of two independent complex Gaussian random variables (equation 5.2.8) results in a complex Gaussian random variable with $E\left[\hat{e}_{1}\right]=0$, and $E\left[\left|\tilde{e}_{1}\right|^{2}\right]=\sigma_{1}^{2}=2 \sigma_{b}^{2}+N_{0}$.

The remaining $\tilde{e}_{i}(i>1)$ are complex Gaussian random variables which can be generated by using some arbitrary orthonormal set $\tilde{\Phi}_{i}(t)(i \neq 1)$ whose members are orthogonal to $\tilde{s}(t)$. Then,

$$
\tilde{e}_{i}=\int_{0}^{T} \tilde{e}(t) \tilde{\Phi}_{i}^{*}(t) d t=\tilde{w}_{i}, \quad i \neq 1 .
$$

Because $\tilde{w}_{i}$ and $\tilde{w}_{j}$ for $i \neq j$ are uncorrelated, and furthermore jointly Gaussian, they are statistically independent. We see that all $\tilde{e}_{i}(i>1)$ are equal to the coefficients of the noise expansion, $\tilde{w}_{i}$, only the first coefficient $\tilde{e}_{1}$ contains information originating from the signal. Further, all $\tilde{e}_{i}(i>1)$ are statistically independent of $\tilde{e}_{1}$, therefore $\tilde{e}_{1}$ is a sufficient statistic. The probability density of $\tilde{e}_{1}$ given the parameter $v$ follows immediately:

$$
p\left(\tilde{e}_{1} \mid v\right)=p\left(\tilde{e}_{1} \mid \tilde{e}_{1}=\tilde{b}+\tilde{w}_{1}\right)=\left(\pi \sigma_{1}^{2}\right)^{-1} \exp \left(-\frac{\left|\tilde{e}_{1}\right|^{2}}{\sigma_{1}^{2}}\right) .
$$


As was mentioned in Section 5.1 the conditional probability density, $p\left(\tilde{e}_{1} \mid v\right)$, defines the likelihood function when the numerical value of $\tilde{e}_{1}$ is inserted. Since we are only interested in the optimal velocity which maximizes the likelihood function, it may be divided by another function that does not depend on its parameter, $v$. We choose to divide it by the probability density of coefficient $\tilde{e}_{1}$ in situation when $\tilde{e}_{1}=\tilde{w}_{1}$, therebry assumed that no signal $\tilde{r}(t)$ is received $\left(p\left(\tilde{e}_{1} \mid \tilde{e}_{1}=\tilde{w}_{1}\right)=\left(\pi N_{0}\right)^{-1} \exp \left(-\left|\tilde{e}_{1}\right|^{2} / N_{0}\right)\right.$. Taking the logarithm and rearranging terms yields the likelihood function $l(v)$ to be of the following form

$$
l(v)=\log \frac{p\left(\tilde{e}_{1} \mid v\right)}{p\left(\tilde{e}_{1} \mid \tilde{e}_{1}=\hat{w}_{1}\right)}=\alpha\left|\tilde{e}_{1}\right|^{2}
$$

Thus the likelihood function is proportional to the squared magnitude of the sufficient statistic. The factor $\alpha$, which is independent of $v\left(\sigma_{1}^{2}\right.$ does not depend on $v$ ), is not of importance in our development since we will be dealing with maximization of $l(v)$ over the variable $v$. Therefore we will discard it from now on. Substitution of equation (5.2.7) into (5.2.11) gives the expression for the likelihood function $l(v)$,

$$
l(v)=\left|\int_{0}^{T} \tilde{e}(t) \tilde{s}^{*}(t ; v) d t\right|^{2} .
$$

The likelihood function depends explicitly on all possible velocities, $v \in \mathcal{V}$. As indicated by (5.1.1) the velocity which gives the largest value of the likelihood should be taken as the estimate. Hence, the corresponding maximum likelihood velocity estimator is

$$
\hat{v}=\arg \max _{v} l(v)
$$

where likelihood $l(v)$ is defined by $(5.2 .12)$. It is a general form of the likelihood function that will be used in our application. The next step is to find an exact form of $\tilde{r}(t)(5.2 .1)$ for the pulsed backscattered signal from blood which can be inserted into the likelihood function.

\subsubsection{Formulation of Likelihood Function for Blood Veloc- ity Estimation}

In this section we formulate the likelihood function for estimating the velocity of blood scatterers. The first step is to develop a mathematical model for the physical situation of interest. Once we obtain the mathematical model, the estimation procedure is directly analogous to that in the previous section, so that we can proceed quickly.

In pulsed measurement mode a received signal consists of a sequence of radiofrequency (RF) signals, separated by a relatively long period $T_{\text {prf }}$. In order to develop a model for the backscattered RF signal we recall the physical properties of the RF signal described in Section 2.4. The total backscattered signal is composed of the sum of the signals returned by a large number of scatterers present in the sample volume. Each scatterer moving at velocity $v_{i}$ induces a delay and attenuates the 
pulse function. Suppose the $i$-th scatterer positioned at depth $z_{i}$ has the scattering strength $A_{i}$ and induces delay given by $2 T_{\text {prf }} v_{i} / c$ according to (2.4.3) for $\cos \theta=1$. The RF signal due to the $k$-th transmission can then be written as:

$$
\begin{aligned}
r(x, k) & =s(x) *\left[\sum_{i} A_{i} \delta\left(x-z_{i}-T_{\mathrm{prf}} v_{i}\right)\right] \\
& =\sum_{i} A_{i} s\left(x-z_{i}-k T_{\mathrm{prf}} v_{i}, 0\right),
\end{aligned}
$$

where $*$ is the convolution operator, $\delta$ the unit-impulse function. The summation in (5.2.14) represents a large number of scatterers of random positions as well as scattering strengths.

Following the derivation from the previous section the complex envelope representation $\tilde{r}(x, k)$ with respect to $x$ is needed. Similar to equation (5.2.14) the Hilbert transform of $r(x, k)$ satisfies the equality

$$
r_{h}(x, k)=\sum_{i} A_{i} s_{h}\left(x-z_{i}-k T_{\mathrm{prf}} v_{i}, 0\right),
$$

where $s_{h}(x)$ is the Hilbert transform of $s(x)$. Using expressions (3.4.1), (5.2.14), and (5.2.15) we obtain that the complex envelope of $r(x, k)$ can be expressed in terms of the complex envelope of $s(x)$ as follows

$$
\begin{aligned}
\tilde{r}(x, k)= & \left(r(x, k)+j r_{h}(x, k)\right) \exp \left(-j 2 \pi \xi_{\mathrm{e}} x\right) \\
= & \sum_{i} A_{i}\left(s\left(x-z_{i}-k T_{\mathrm{prf}} v_{i}, 0\right)+j s_{h}\left(x-z_{i}-k T_{\mathrm{prf}} v_{i}, 0\right)\right) \exp \left(-j 2 \pi \xi_{\mathrm{e}} x\right) \\
= & \sum_{i} A_{i}\left(s\left(x-z_{i}-k T_{\mathrm{prf}} v_{i}, 0\right)+j s_{h}\left(x-z_{i}-k T_{\mathrm{prf}} v_{i}, 0\right)\right) \\
& \times \exp \left(-j 2 \pi \xi_{\mathrm{e}}\left(x-z_{i}-k T_{\mathrm{prf}} v_{i}\right)\right) \exp \left(-j 2 \pi \xi_{\mathrm{e}}\left(z_{i}+k T_{\mathrm{prf}} v_{i}\right)\right) \\
= & \sum_{i} A_{i} \tilde{s}\left(x-z_{i}-k T_{\mathrm{prf}} v_{i}, 0\right) \exp \left(-j 2 \pi \xi_{\mathrm{e}}\left(z_{i}+k T_{\mathrm{prf}} v_{i}\right)\right) .
\end{aligned}
$$

In order to develop our model of the backscattered signal in the form similar to equation (5.2.1) we have to apply to the result of (5.2.16) the "slowly fluctuating point target" approximation, commonly used in radar. Slowly fluctuating point target approximation models the groups of scatterers within a sample volume as a point target moving at a constant velocity $v$. We now want to determine the characteristics of the sum in (5.2.16) for a point target with several reflecting surfaces $i=1, \ldots, K$. If we assume that the random phase $z_{i}$ incurred in the reflecting process are statistically independent, that the reflecting strengths $A_{i}$ have equal magnitudes, and that $K$ is large, we can use a central limit theorem argument to obtain

$$
\tilde{r}(x, k)=\tilde{b} \tilde{s}\left(x-z-k T_{\mathrm{prf}} v, 0\right) \exp \left(-j 2 \pi \xi_{\mathrm{e}} k T_{\mathrm{prf}} v\right),
$$

where the multiplier $\tilde{b}$ may be considered as a complex Gaussian random variable. It includes the effect of summation over scatterers of the product of the signal amplitude and the random phase of each scatterer $\left(\tilde{b}=\sum_{i} A_{i} \exp \left(-j 2 \pi \xi_{\mathrm{e}} z_{i}\right)\right)$. The distance of 
the target from the transducer is given by depth $z$, therefore the transmitted signal acquires the delay of $z$.

The mathematical model of the RF signal due to the $k$-th transmission (equation 5.2.17) compared to the general model description (equation 5.2.1) is of the same form we were seeking. Therefore, a similar argument to that in Section 5.2 .1 can be applied. We can expand the signal (5.2.17) corrupted by noise $(\tilde{e}(x, k)=\tilde{r}(x, k)+$ $\tilde{w}(x, k))$ as given by (5.2.3) using any complete orthonormal set of functions and obtain statistically independent coefficients. Just as in the previous section, we can choose the signal $\tilde{s}(x)$ as the first orthonornal function and the resulting coefficient will be a sufficient statistic. Since the signal $\tilde{s}(x)$ consists of a sum of short pulses, the sufficient statistic as a function of parameter $v$ would then be

$$
\tilde{e}_{1}=\sum_{k} \int_{0}^{T} \tilde{e}(x, k) \tilde{s}^{*}\left(x-z-k T_{\mathrm{pr}} v, 0\right) \exp \left(j 2 \pi \xi_{\mathrm{e}} k T_{\mathrm{prf}} v\right) d x .
$$

Since we are considering only a particular value of $z$, we can assume that it is zero for algebraic simplicity. The modification for nonzero $z$ is obvious. The likelihood function $l(v)$ for $v \in \mathcal{V}$ then follows from equation (5.2.11)

$$
l(v)=\left|\sum_{k} \int_{0}^{T} \tilde{e}(x, k) \tilde{s}^{*}\left(x-k T_{\mathrm{prf}} v, 0\right) \exp \left(j 2 \pi \xi_{\mathrm{e}} k T_{\mathrm{prf}} v\right) d x\right|^{2} .
$$

In words, first the particular received signal $\tilde{e}(x, k)$ is matched with the complex conjugated model of the noiseless signal $\tilde{s}\left(x-k T_{\mathrm{prf}} v, 0\right) \exp \left(j 2 \pi \xi_{\mathrm{e}} k T_{\mathrm{prf}} v\right)$ giving a sample of correlation for a velocity $v$ at a particular time $k$. Then a number of correlation samples are averaged over time. Finally, taking its squared magnitude yields the value of the likelihood function at a velocity $v$. This procedure is repeated for all possible velocities, $v \in \mathcal{V}$. The estimate of the velocity then follows from $(5.2 .13)$.

The form (5.2.19) of the likelihood function is identical to the expression used in Ferrara and Algazi in [20], and slightly different from the expression of Ferrara and Algazi in [19]. The difference is in the exponential term. It is possible to derive (5.2.19) from formula 6 in [19] where inside the exponential function the additional term $j 2 \pi \xi_{\mathrm{e}} v x$ is found. Estimates based on both forms of the likelihood function were compared in [65] and it was concluded that a difference is not evident.

\subsubsection{Implementation of Maximum Likelihood Estimator}

In this section we describe the implementation of the identification procedure given by the maximum likelihood method. It involves the processing scheme of the maximum likelihood estimation, and a choice of a reference function in the likelihood expression (5.2.19).

For maximum likelihood velocity estimation we must generate the likelihood funs tion (5.2.19) for all values of $v$ in the region of interest. For any particular $v \in \mathcal{V}$, 


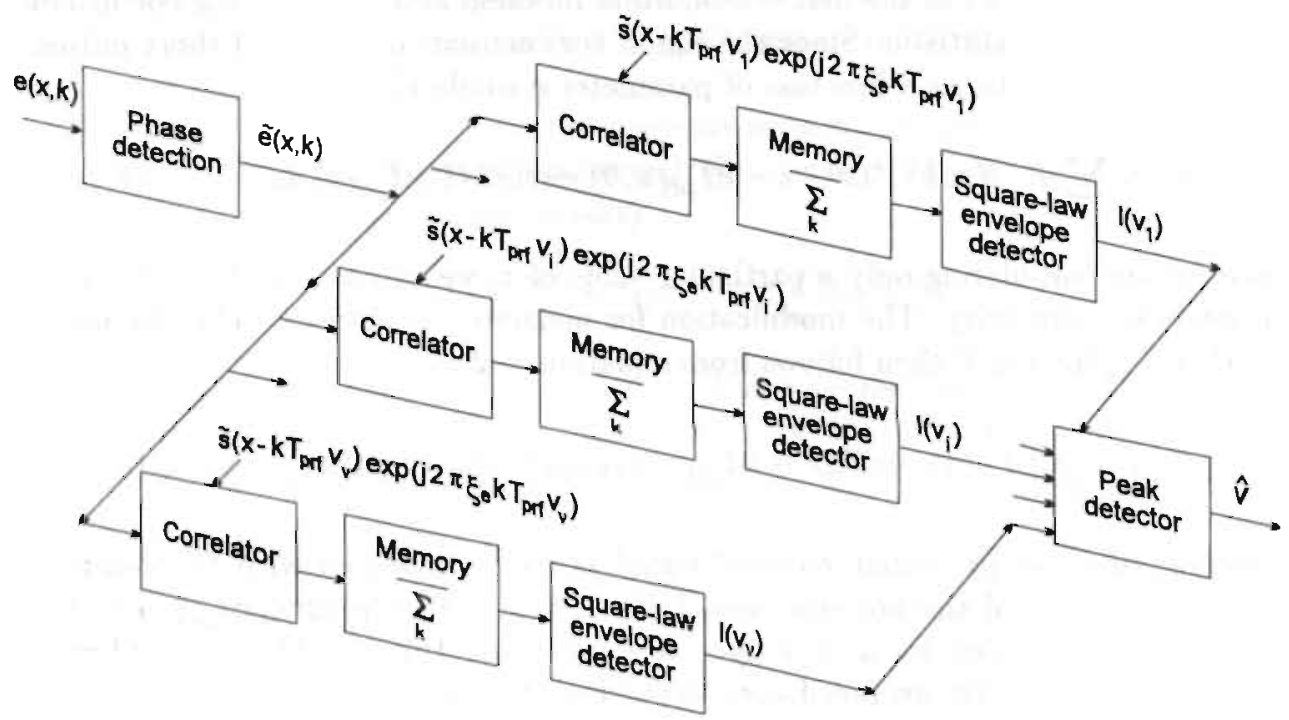

Figure 5.1. Scheme for maximum likelihood estimation of blood velocities. A transformed backscattered signal is correlated in depth with a reference signal tuned to a possible velocity for $i=1, \ldots, \nu$. Notice, that first block - Correlator, and the second - Memory $\mid \sum_{k}$, form together the realization of two-dimensional correlation of the backscattered and reference signal at lag given by $\left(k T_{\mathrm{prf}} v_{i}, 0\right)$. The magnitude squared of the complex valued correlation gives the likelihood value for a particular velocity. Each parallel processing line is tuned to a different velocity so that they cover all possible velocities present in the backscattered signal. The last block of this scheme detects the peak of discrete likelihood function and the estimate of velocity is chosen as that value of velocity which gives this peak. 

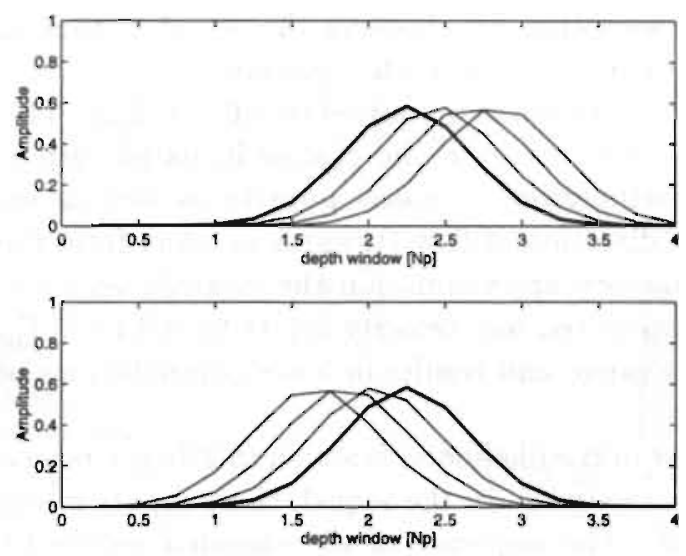

FIGURE 5.2. Reference signal within depth window of 4 periods. The top depicts the movement for positive simulated velocities, the bottom shows it for negative velocities. The starting position corresponding to the 0 -th transmission is indicated in bold line. It should be noticed that it is an overlap of four subsequent reference sig. nals tuned to scaled velocity $\vartheta= \pm 0.2 \lambda / T_{\text {prf }}$. The quality factor of the transmitted pulse was chosen to be $Q_{\mathrm{s}}=2$.

we can generate it using a correlation receiver or matched filter. Our realization uses a correlation receiver which is easy to implement on a $\mathrm{PC}$. The maximum likelihood procedure is illustrated schematicaly in Fig. 5.1. The likelihood function is realized as its discrete approximation for possible values $v_{i}, i=1, \ldots \nu$ that span the velocity range of interest. Therefore the scheme contains $\nu$ parallel processing lines.

A backscattered signal due to the $k$-th transmission, $e(x, k)$, is transformed into the complex envelope signal $\tilde{e}(x, k)$ with respect to the frequency $\xi_{\mathrm{e}}$. This transformation was described in Section 3.4 and causes a shift of the spectral content of a signal to the lower frequencies. Hence, the maximum likelihood estimator is based on the processing of the low frequency signal $\tilde{e}(x, k)$, for $k=1, \ldots, M$. The sampled (low frequency) signal $\tilde{e}(x, k)$ is passed through $\nu$ parallel branches, where $\nu$ is a number of possible velocities, $v_{i}, i=1, \ldots, \nu$. In each branch it is correlated with a reference signal $\tilde{s}\left(x-k T_{\mathrm{prf}} v_{i}, 0\right) \exp \left(j 2 \pi \xi_{\mathrm{e}} k T_{\mathrm{prf}} v_{i}\right)$ tuned to a particular velocity $v_{i}$. Correlations obtained from $M$ successive RF returns are stored and subsequently averaged. In fact a two-dimensional correlation of the backscattered signal and the reference signal at a particular lag is performed at each processing line.

The likelihood function for a particular velocity is obtained as the squared absolute value of the correlation. The discrete approximation of the function $l(v)$ depends explicitly on considered velocity components. The position of the maximum of the likelihood function indicates the most likely velocity of blood which is then taken as its estimate, $\hat{v}$. 
We have just seen that by choosing the set of $v_{i}$ that span the velocity range of interest, we can obtain a discrete approximation to $l(v)$ as indicated in figure 5.1. The scaled velocity range is limited to $\pm 0.5 \lambda / T_{\text {prf }}$. The reason for this is that aliasing (Section 2.4.4) occurs if the change in pathlength is greater than half the wavelength $\lambda$. Furthermore, it spans positive as well as negative velocities. The sign indicates the direction of flow (towards or away from the transducer). In order to obtain a satisfactory approximation the (scaled) velocity grid was chosen to be $0.01 \lambda / T_{\text {prf }}$. We restricted our velocity limits to $\pm 0.45 \lambda / T_{\text {prf }}$ which covers almost the whole velocity range and results in fewer computations per estimate.

Two signals appear in the likelihood function (5.2.19): a received signal in its complex form and a reference signal. In the sequel, these inputs of our simulation procedure will be considered. The sequence of the sampled received signals $e(x, k)$ for $k=$ $0, \ldots, M-1$ forming the data matrix $\mathbf{D}(M, N)$ in figure 3.1 is generated according to the simulation model of Chapter 4 . In order to obtain a low frequency signal $\tilde{e}(x, k)$, a complex envelope transformation (equation 3.4.1) is applied to each $e(x, k)$ for $k=0, \ldots, M-1$. The low frequency signals are then processed in the way indicated by the maximum likelihood scheme of figure 5.1. We want to emphasize that for the signals generated by our simulation procedure the slowly fluctuating point target approximation is not valid. Although the likelihood function has been derived under this approximation, we apply the simulated signals that do not satisfy this assumption and we investigate the behavior of the method.

The reference signal for the ML scheme will be characterized now. According to Assumption 4.1, the envelope of the transmitted signal (and therefore also the reference signal) is supposed to have a Gaussian shape, in concrete terms,

$$
\begin{aligned}
s(x) & =\exp \left(-\frac{x^{2}}{2 x_{d}^{2}}\right) \cos \left(2 \pi \xi_{\mathrm{e}} x\right) \\
& =\operatorname{Re}\left[\exp \left(-\frac{x^{2}}{2 x_{d}^{2}}\right) \exp \left(j 2 \pi \xi_{\mathrm{e}} x\right)\right],
\end{aligned}
$$

where $x_{d}$ is the duration of the signal. Comparison of right-hand sides of (5.2.20) and (3.4.2) implies that the complex. envelope of reference signal should be chosen as

$$
\tilde{s}(x-\Delta x, 0)=s_{0}(\Delta x) \exp \left(-\frac{(x-\Delta x)^{2}}{2 x_{d}^{2}}\right), \quad \Delta x=k T_{\mathrm{prf}} v_{i},
$$

where $s_{0}(\Delta x)$ is constant for fixed $\Delta x$. Because of the condition (5.2.5) on the orthonormal function, the reference signal has to be modified through $s_{0}(\Delta x)$. So that

$$
\int_{0}^{T}|\tilde{s}(x-\Delta x)|^{2} d t=1,
$$

for all $\Delta x$ the energy does not depend on the shift. The value of $x_{d}$ can be extracted from the inverse Fourier transform of equation (4.2.1), resulting in $x_{d}=$ 


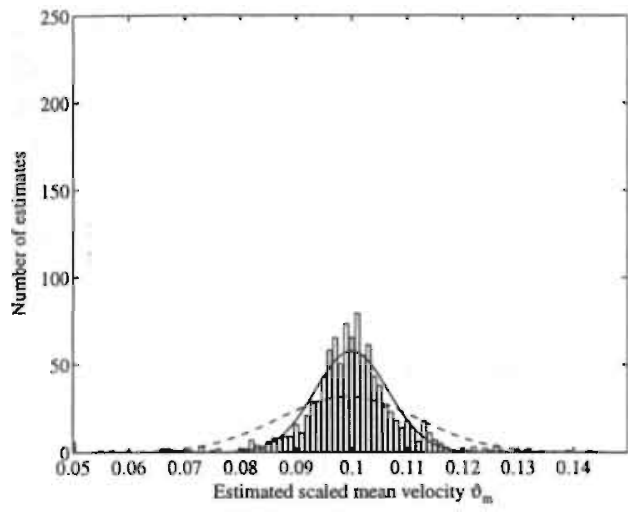

(a)

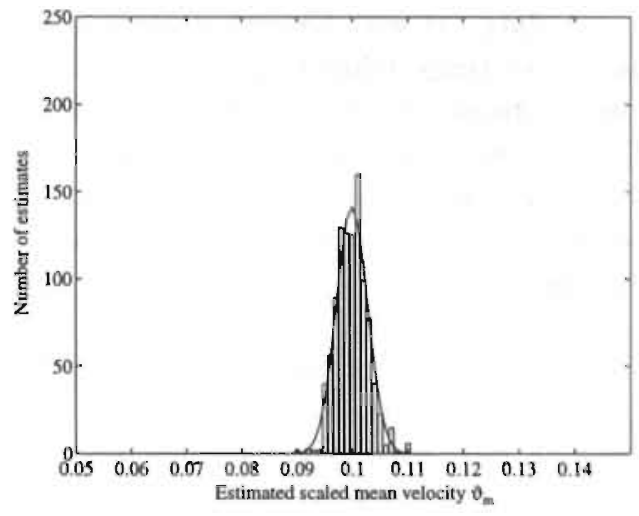

(b)

FIgURE 5.3. Two histograms of maximum likelihood estimates with approximated distribution function. (a) shows the unfitered estimates, (b) depicts the maximum likelihood estimates filtered by a median filter. The model parameters are fixed to the following values: $M=25$ observations, $N_{\mathrm{p}}=8$ periods, $\vartheta_{\mathrm{m}}=0.1 \lambda / T_{\mathrm{prf}}, \vartheta_{\mathrm{s}}$ $=0, Q_{\mathrm{rf}}=2, \mathrm{SNR}=10 \mathrm{~dB}$.

$\sqrt{\log 2} Q_{\mathrm{s}} / 4 \xi_{\mathrm{e}}$. Two input parameters, quality factor $Q_{\mathrm{s}}$, and center frequency $\xi_{\mathrm{e}}$, are matched to the values used for simulation of the backscattered signal in Chapter 4. The quality factor $Q_{\mathrm{s}}$ equals the quality factor $Q_{\mathrm{rf}}$ (Section 4.2).

The generated signal for the 0 -th transmission $\tilde{s}(x, 0)$ is placed in the center of the sample volume and shifted with each observation $(k>0)$ according the $\Delta x$ as indicated in figure 5.2. In this figure a model is generated for signals with quality factor $Q_{s}=2$ within a window of 4 periods. Four subsequent signals are shifted to the right for positive mean velocity $\left(\vartheta_{\mathrm{m}}=0.2 \lambda / T_{\mathrm{prf}}\right)$, and to the left for negative mean velocity $\left(\vartheta_{\mathrm{mn}}=-0.2 \lambda / T_{\mathrm{prf}}\right)$. It should be mentioned that these plots are illustrative, to indicate the shift between signals due to the motion. Actually, just one signal within a depth window enters the correlator of figure 5.1 at time $k$.

At this stage, all quantitative input parameters of the simulation procedure for maximum likelihood estimator of blood velocity are known, so the likelihood function (5.2.19) can be constructed and the velocity estimate (5.2.13) identified as shown in figure 5.1 .

\subsection{Statistical Properties of the Maximum Likelihood Es- timator}

From classical estimation theory [43] we know that the maximum likelihood estimator converges in distribution to the normal distribution. The exact analytical calculation of asymptotic properties (as the sample size, number $S$, tends to infinity) is hindered by the complexity involved. Therefore, we have chosen to address it by means of Monte Carlo simulation study with a sample set of $S=1000$ estimates. 
A data set was generated according to the simulation model of Chapter 4 with model settings defined by equation (4.3.3) and described in Section 4.3, for $M=25$ observations, $N_{\mathrm{p}}=8$ periods, $\vartheta_{\mathrm{m}}=0.1 \lambda / T_{\mathrm{prf}}, \vartheta_{\mathrm{s}}=0, Q_{\mathrm{rf}}=2, \mathrm{SNR}=10 \mathrm{~dB}$. The $S$ simulation runs were made independently for the identical conditions. The likelihood function was implemented as described in Section 5.2.3, however, here with (scaled) velocity grid of $0.001 \lambda / T_{\mathrm{prf}}$ for fine approximation of the likelihood function.

The distribution of the maximum likelihood estimates of the imposed scaled mean velocity $\vartheta_{\mathrm{m}}=0.1 \lambda / T_{\mathrm{prf}}$ is plotted in figure 5.3 (a). At first sight, the estimates look to be normally distributed around their mean $\left(0.1 \lambda / T_{\text {prf }}\right)$. An attempt to fit a Normal curve to their distribution in Fig. 5.3 (a) is given by two lines. The dashed Gaussian curve with mean 0.0993, and standard deviation 0.0126 estimated from the whole sample, does not exactly overlap with the distribution of estimates. It indicates that estimates are broadly spread around their mean. Nevetherless, if we consider only estimates lying in the interval between 0.08 and $0.012 \lambda / T_{\text {prf }}$, the resulting solid Gaussian curve in Fig. 5.3 (a) gives a good approximation of the distribution function. It happens that $95.9 \%$ of estimates lie within the given interval. Their mean 0.0999 , and standard deviation 0.0066 have been considerably improved. Note, that the standard deviation is $50 \%$ lower than the one obtained from the whole sample.

The reason for the difference in standard deviation is an occasional large measurement error which causes the sequence of estimates to consist of strong, spikelike components. The corresponding abnormal data points are called outliers and give: large contributions to the estimates of mean value (3.3.6) and standard deviation (3.3.7). In the following we will give insight into the causes of this type of error.

As we know, the estimation of the velocity by the maximum likelihood method is done by finding the position of the maximum in the likelihood function. Thus the reliability of this nonlinear approach depends on the quality of the likelihood function. The question is whether the peak of the likelihood function occurs at the correct position which gives the velocity estimate. As pointed out in Section 5.2.3, the characteristics of applied simulated signals differ from the slowly fluctuating point target characteristics. This approximation was used in the derivation of the likelihood function in Section 5.2.2 and causes mismatch between the simulated backscattered signal and reference signal. The incompatibility between the assumption and actual characteristics of the applied signal is a reason of misplacement of the peak of the likelihood function that lead to large identification errors. This error is very significant at poor signal-to-noise ratios. The noise contribution increases the decorrelation between the subsequent signals, whereas almost "perfect" correlation is assumed for the reference signal.

An example of the likelihood function of an individual estimate for poor and high signal to noise ratios is plotted in Fig. 5.4. The following setting was simulated: $M$ $=25$ observations, $N_{\mathrm{p}}=4$ periods, $\vartheta_{\mathrm{m}}=0.3 \lambda / T_{\mathrm{prf}}, Q_{\mathrm{rf}}=4$. The other parameters 


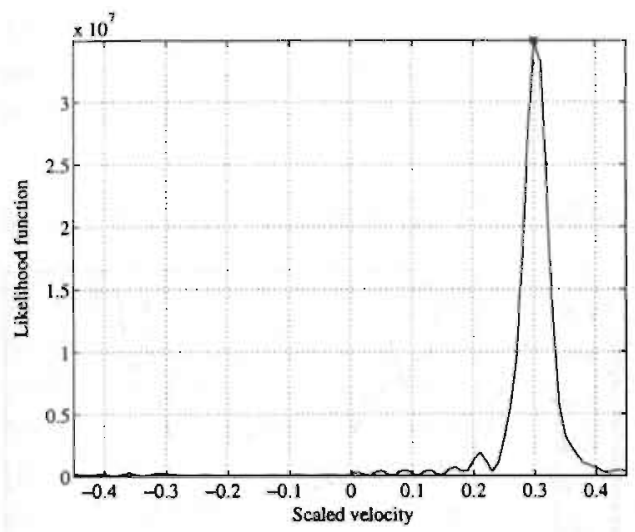

(a)

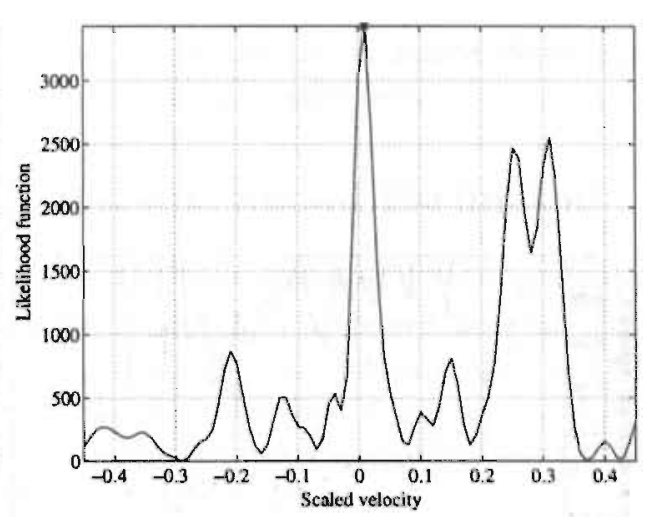

(b)

Figure 5.4. Examples of likelihood function. The star in the plot indicates the detected peak. Mlustrative simulations with (a) SNR $=40 \mathrm{~dB}$, (b) SNR $=0 \mathrm{~dB}$. The other model parameters are kept constant: $M=25$ observations, $N_{\mathrm{p}}=4$ periods, $\vartheta_{\mathrm{m}}=$ $0.3 \lambda / T_{\text {prf }}, \vartheta_{\mathrm{s}}=0, Q_{\mathrm{rf}}=4$.

are identical to those in Fig. 5.3. It is clear that the likelihood function is a positive function whose maximum value is located at a velocity the scatterers are suppose to move with. However, due to noise in the acquired signals and the use of data whose characteristics are more complicated from those assumed, it is quite possible that the maximum will be at a position different from that which the true velocity would indicate. In Fig. 5.4 (a), the shape of the likelihood function is reasonably flat except for the central peak that appears at the correct value, $0.3 \lambda / T_{\text {prf }}$ (simulated under SNR of $40 \mathrm{~dB}$ ). On the other hand, Fig. 5.4 (b) where $\mathrm{SNR}=0 \mathrm{~dB}$, demonstrates the harmful effect of noise on the likelihood function. We observe that the noise contribution caused the total value at the subsidiary peak to exceed the value at the major peak. As a consequence, a false estimate of the mean velocity will be obtained that will appear as a "spike" in the sequence of estimates.

To the velocity estimates, a nonlinear postprocessing technique like median filtering may be applied to improve their quality [6], [35]. The outliers can be filtered out and the behavior of the maximum likelihood estimate can be smoothed to some extent. The effect of median filtering on the velocity distribution can be seen in figure 5.3 (b), where a median filter of 7-th order was applied to estimates. In this case the distribution of the filtered estimates follows a Gaussian curve with mean 0.0999 , and standard deviation 0.0028 , estimated from filtered data. Note that the standard deviation is improved by almost $80 \%$. Median filtering ( 7 -th order) of estimates based on 25 observations is in practice comparable to the situation that uses $7 \cdot 25=175$ returns for one velocity estimate. Then the applicability of high order median filtering is questionable when we realize that the RF signal is only temporarily stationary. We included the median filtering in order to show that the maximum likelihood velocity estimates can be improved to some extent by such postprocessing. 


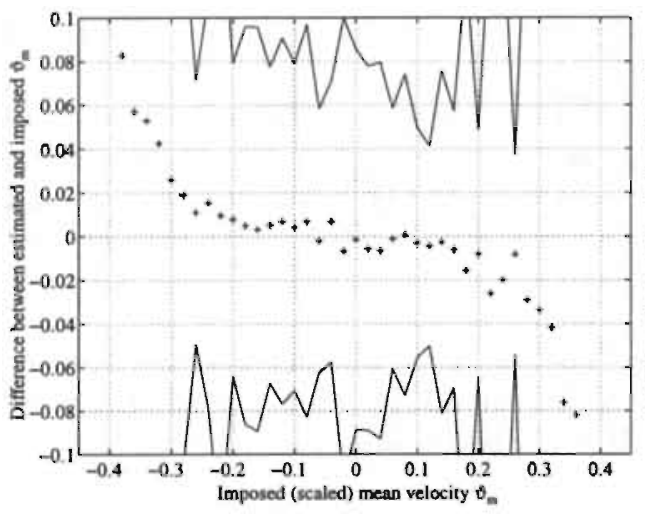

(a)

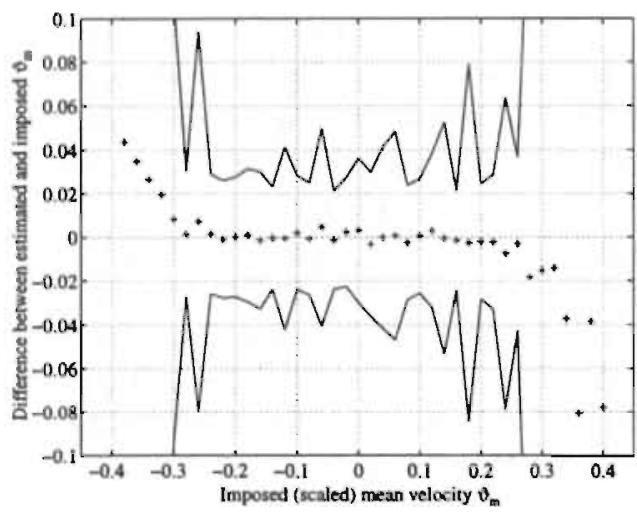

(c)

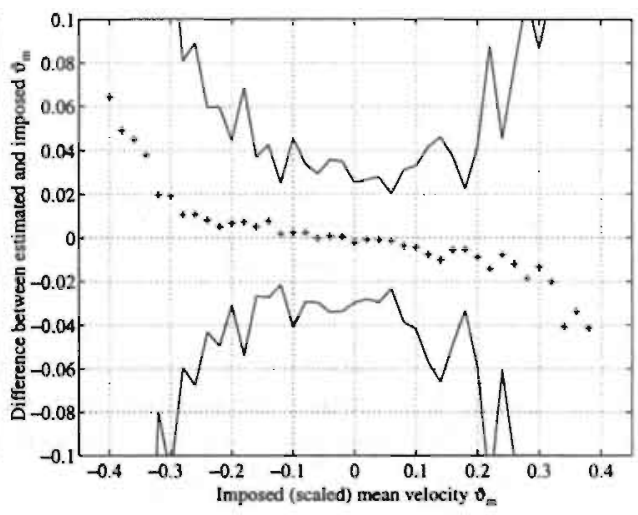

(b)

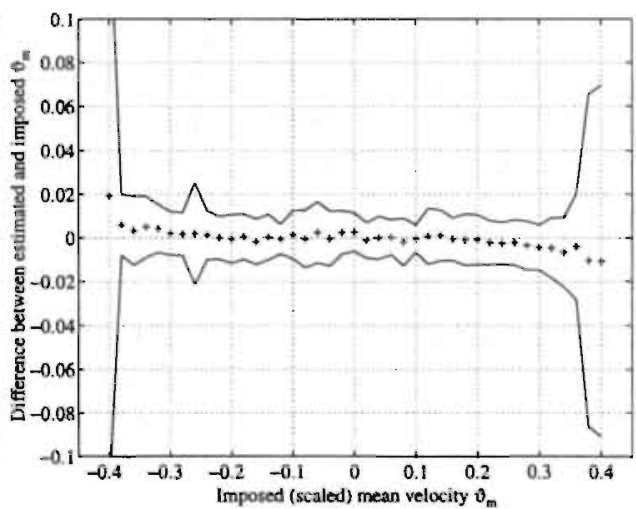

(d)

FIGURE 5.5. Difference between estimated and imposed scaled mean velocity, $\vartheta_{m}$, with the $95 \%$ confidence interval. (a) $N_{\mathrm{p}}=4$ periods, $M=8$ observations, (b) $N_{\mathrm{p}}$ $=4$ periods, $M=16$ observations, (c) $N_{\mathrm{p}}=8$ periods, $M=16$ observations, (d) estimates from $(c)$ filtered by 7 -th order median filter. The other model parameters are kept constant: $\vartheta_{\mathrm{S}}=0, \mathrm{SNR}=10 \mathrm{~dB}, Q_{\mathrm{rf}}=2$. 
The conclusion that could be drawn is that we can consider the ML estimates to be assymptotically normally distributed. Therefore, the mean and standard deviation will sufficiently describe the sample distribution of the ML estimates.

\subsection{Performance of the Maximum Likelihood Estimator}

To examine the behavior of the maximum likelihood estimator and to investigate its practical potential, the computer simulation approach is preferred because this problem cannot be addressed so easily by purely analytical methods, due to the complexity involved. In case of the maximum likelihood method it interests us how the incompatibility between assumed model of the backscattered signal and the signal actually applied influences the performance of the method under various processing and system parameters.

A data set is generated according to the simulation model of Chapter 4 for the settings defined by (4.3.3), and described in Section 4.3. The implementation of the likelihood function follows the description given in Section 5.2.3. For computational reasons, we reduced the sample set to $S=100$ estimates in comparison to the sample set used for analysis in the previous section where $S$ was set to 1000 .

Since the (imposed) properties of the simulated signals are completely known a qualitative assessment of the performance of the ML estimator is possible. This task is evaluated in terms of the bias and standard deviation of resulted estimates obtained from a sample set $S$ according to equations (3.3.8), and (3.3.7).

In the following subsections we investigate the effect of numerous different parameters on the performance of the ML estimator individually. In Section 5.4.1 we study the influence of the scaled mean velocity, in Section 5.4 .2 we inquire into the effects of sizes of the temporal and spatial windows, and into the effect of velocity dispersion. In Section 5.4.3 we pay attention to the influence of the signal-to-noise ratio and quality factor. In the last Section 5.4.4 we summarize the observations obtained from the computer simulation results.

\subsubsection{Effect of Velocity}

Let us inspect the behavior of the ML estimator as a function of scaled mean velocity $\vartheta_{\mathrm{m}}$. The difference between imposed and estimated scaled mean velocity (bias) is plotted as plus sign in Fig. 5.5 for the velocities within the range of $(-0.4,0.4) \lambda / T_{\mathrm{prf}}$ with step of $0.02 \lambda / T_{\mathrm{prf}}$. Around the bias values, a $95 \%$ confidence interval (two times the standard deviation) is drawn, which provides information about the distribution of estimates at a particular velocity. All parts of Fig. 5.5 (a), (b), (c), and (d) are generated with zero velocity spread, quality factor $Q_{\mathrm{rf}}=2$, and $S \mathrm{NR}=10 \mathrm{~dB}$. Window length in depth and in time differ per plot.

Following the sequence of plots in Fig. 5.5 from (a) to (d) the improvement is visible in each step. We will start with Fig. 5.5 (a). The results are obtained for a 


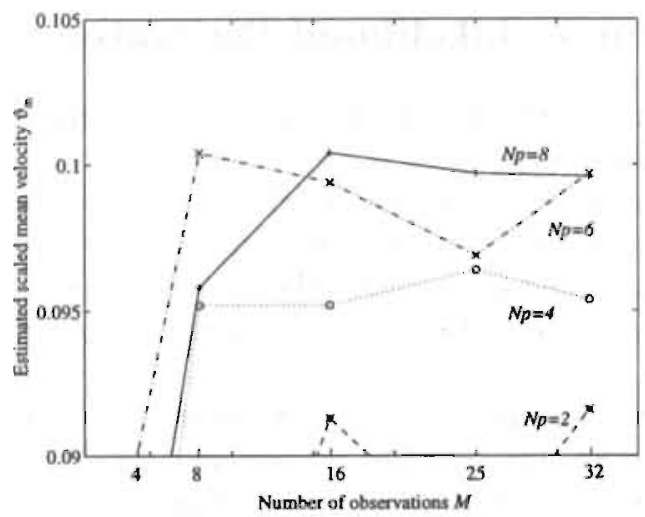

(a)

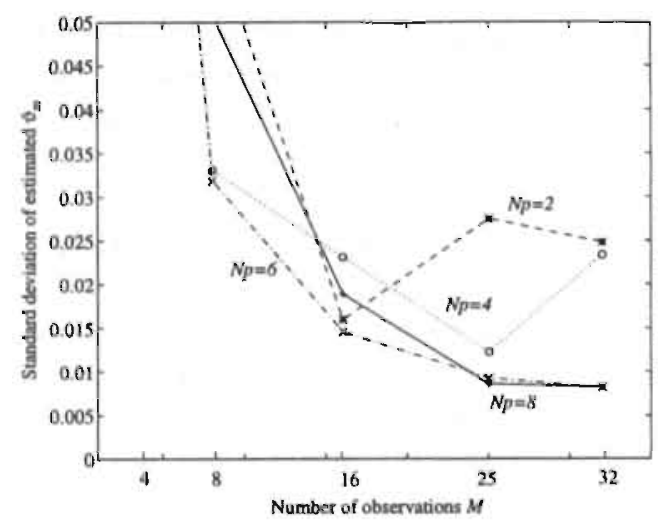

(c)

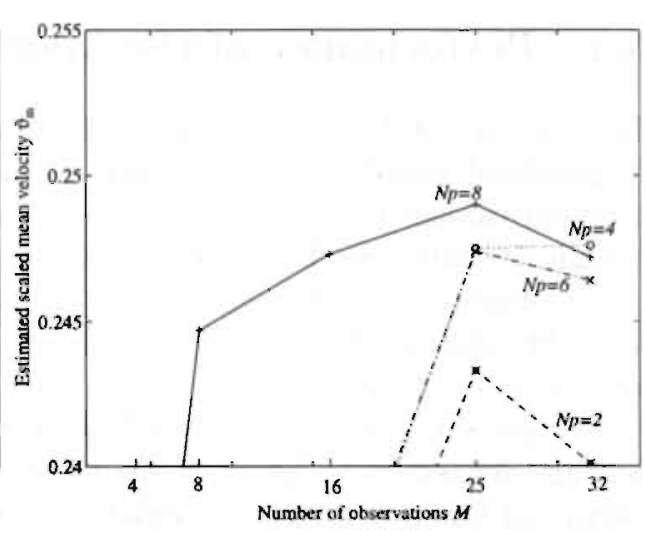

(b)

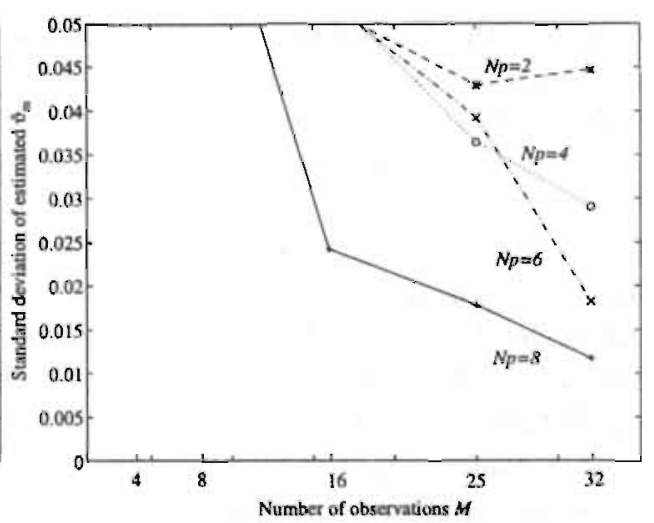

(d)

FIGUAE 5.6. Influence of the depth and time window sizes. Four different lines represent four various sizes of the depth window: the dashed line with stars pertains to $N_{\mathrm{p}}$ $=2$, the dotted line with circles to $N_{\mathrm{p}}=4$, the dashed-dotted line with crosses to $N_{\mathrm{p}}=6$, and the solid line with plus signs to $N_{\mathrm{p}}=8$. The estimates of $\hat{\vartheta}_{\mathrm{m}}$ and standard deviations are plotted for two imposed scaled mean velocities: (a), (c) $v_{\mathrm{mi}}=0.1 \lambda / T_{\mathrm{prf}},(\mathrm{b}),(\mathrm{d}) \vartheta_{\mathrm{m}}=0.25 \lambda / T_{\mathrm{prf}}$ as function of the time window size of $M=4,8,16,25$, and 32 observations. The other model parameters are kept constant: $\vartheta_{\mathrm{s}}=0, Q_{\mathrm{rf}}=2, \mathrm{SNR}=10 \mathrm{~dB}$. 
depth window of 4 periods, and time window of 8 observations. The bias exceeds the value of $|0.01| \lambda / T_{\text {prf }}$ for scaled mean velocities, $\left|\vartheta_{\mathrm{m}}\right|>0.2 \lambda / T_{\text {prf }}$, reaching the value of about $|0.04| \lambda / T_{\text {prf }}$ for scaled mean velocities around $|0.3| \lambda / T_{\text {prf }}$. Also the $95 \%$ confidence interval exceeds the extreme values - $|0.1| \lambda / T_{\text {prf }}$ for some velocities. The values are outside the observation window.

The next plot, Fig. 5.5 (b), illustrates the situation when the number of observations per estimate is doubled $(M=16)$. The bias for velocities around zero is smoother and the width of the $95 \%$ confidence interval is decreased by a factor of two approximately. However, the increase in the number of observations has brought no improvement to the statistics for higher (absolute) velocities. Elongating the window length in depth simultaneously, $N_{\mathrm{p}}=8$ periods, as seen in Fig. 5.5 (c), improves the statistics for a broader range of velocities. It can be expected that a further increase in $M$ and $N_{\mathrm{p}}$ will result in a further lowering of bias and narrower confidence intervals for a larger range of scaled velocities. While this trend is encouraging, it should be emphasized that a long depth window is a poor alternative because it causes a loss in spatial resolution. Also the number of observations, $M$, is limited by the temporal stationarity of the RF signal. That is why small depth windows and a low number of observations are desirable.

In Figs. 5.5 (a), (b), (c) we observe the abrupt changes on the border of the confidence interval. We remember from the discussion in Section 5.3 that the ML estimator suffers from large variations in the standard deviation. These variations are due to improper localization of the peak of the likelihood function yielding outliers. In the measurement sequence they appear as discontinuities, making them easy to detect and to remove by a median filter as we have already pointed out in Section 5.3. To outline this fact we plotted in Fig. 5.5 (d) the statistics from filtered estimates. The same data set at each imposed mean velocity was used as for Fig. 5.5 (c), on which the 7 -th order median filter was applied. The abrupt changes in statistics are smoothed for a broad range of scaled velocities. These improvements should be evaluated with caution since a 7 -th order median filter may not be applicable due to the limited stationarity of the RF signal. Despite this postprocessing procedure, the quality of the estimator is not excellent. There is still some bias especially at high velocities. Furthermore, the limits of the $95 \%$ confidence interval indicate that estimates are spread more than $0.01 \lambda / T_{\text {prf }}$ around the mean value. It is again attributed to the slowly fluctuating point target approximation. This assumption is opposed in character to the simulated signal and particularly at high velocities the slow fluctuation of a target is violated.

Highly biased estimates at points close to the limits of our assumed velocity range (between $\pm 0.4 \lambda / T_{\mathrm{prf}}$ ) indicate that the method is highly dependent also on the limits of velocity region considered $\left(|0.45| \lambda / T_{\text {prf }}\right.$ as indicated in Section 5.2.3). The velocities at the positions that correspond to the middle of the assumed velocity space are unbiased. In our situation when the limits of the velocity range are $\pm 0.45 \lambda / T_{\text {prf }}$, we can conclude that the ML estimator does not provide a reliable estimate for scaled mean velocities $\left|\vartheta_{\mathrm{m}}\right|>0.2 \lambda / T_{\mathrm{prf}}$. 


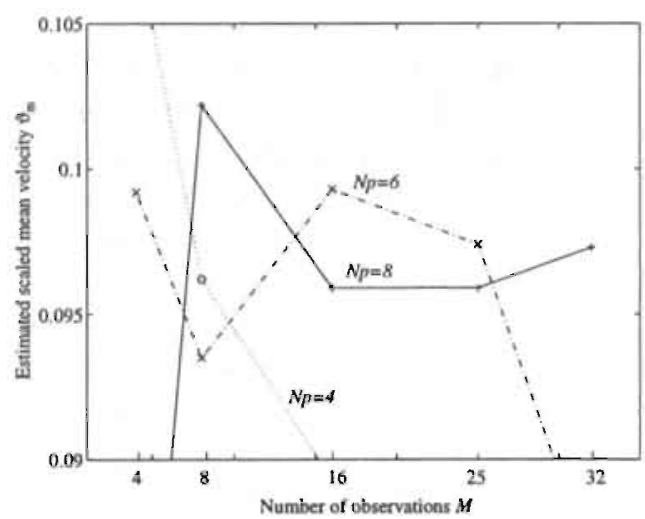

(a)

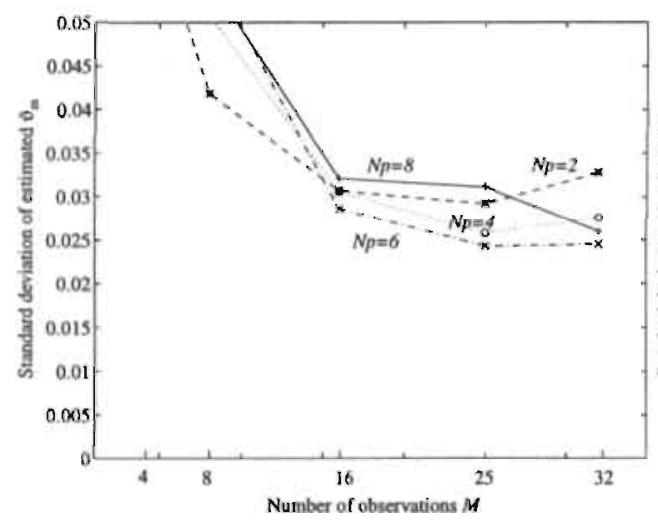

(c)

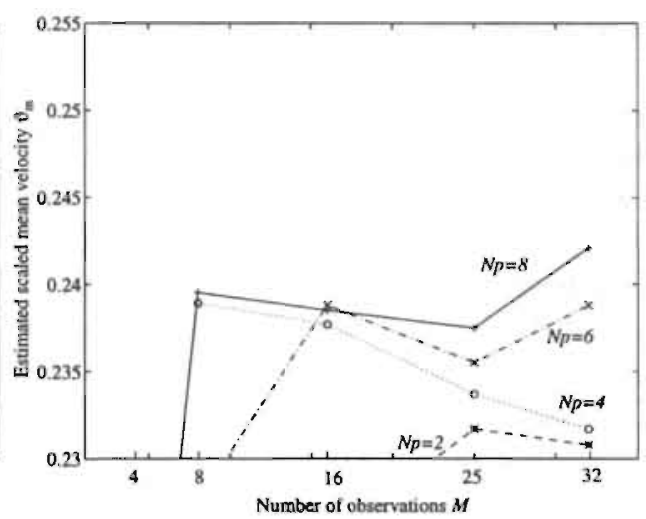

(b)

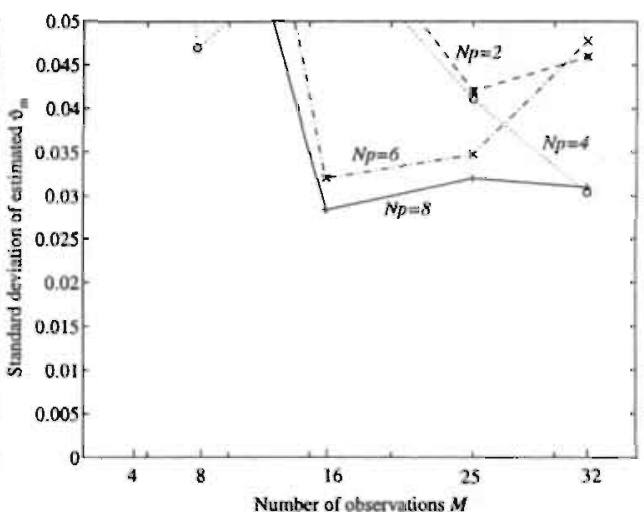

(d)

FigURE 5.7. Effect of velocity spread, $\vartheta_{\mathrm{s}}=0.1 \lambda / T_{\text {prf }}$. Four different lines represent four various sizes of the depth window: the dashed line with stars pertains to $N_{\mathrm{p}}=2$, the dotted line with circles to $N_{\mathrm{p}}=4$, the dashed-dotted line with crosses to $N_{\mathrm{p}}=$ 6 , and the solid with plus signs to $N_{\mathrm{p}}=8$. The estimated scaled mean velocity $\hat{\vartheta}_{\mathrm{m}}$ and their standard deviations are plotted for two imposed scaled mean velocities: (a), (c) $\vartheta_{\mathrm{m}}=0.1 \lambda / T_{\mathrm{prf}}$, (b), (d) $\vartheta_{\mathrm{m}}=0.25 \lambda / T_{\mathrm{prf}}$ as function of the time window size of $M=4,8,16,25$, and 32 observations. The other model parameters are not modified: $Q_{\mathrm{rf}}=2, \mathrm{SNR}=10 \mathrm{~dB}$. 
The application of this algorithm (as described in [19]) reported in [22] has been evaluated only for estimation of low blood velocities in small vessels. There it was claimed that velocities below $1 \mathrm{~mm} / \mathrm{s}$ (which is for their settings approximately $0.05 \lambda / T_{\text {prf }}$ ) can be estimated reliably when using 20 pulses in each estimate. The velocity range and the length of the depth window could not be deduced from the article, so it is quite difficult to compare. However, it shows the limited applicability of this method since only low velocities were investigated.

\subsubsection{Effect of the Processing Parameters}

In the previous section we have already indicated that processing parameters, like the length of the window in depth and time, might have influence on the performance of the ML estimator. In this section we will investigate this dependence for low and high scaled mean velocities and also the effect of nonzero velocity bandwidth.

The simulation experiments were repeated for two imposed scaled mean velocities, corresponding to low and high values. The scaled mean velocity of $0.1 \lambda / T_{\mathrm{pr}}$ represents low velocities, and $0.25 \lambda / T_{\text {prf }}$ is representative of high velocities. Quality factor $Q_{\mathrm{rf}}=2$, and $\mathrm{SNR}=10 \mathrm{~dB}$ were chosen as defaults for all simulations in this section. The estimated mean values are plotted at the top, (a) and (b), while the standard deviations of the estimates are given at the bottom, (c) and (d), of Figs. 5.6, 5.7, and 5.8. They are presented as a function of $M=4,8,1.6,25$, and 32 observations and as a function of the depth window length $N_{\mathrm{p}}=2,4,6$, and 8 periods. The separate lines connect statistics for fixed $N_{\mathrm{p}}$ : the dashed line links stars that pertain to $N_{\mathrm{p}}=2$, the dotted line connects circles for $N_{\mathrm{p}}=4$, the dashed-dotted line relates the crosses for $N_{\mathrm{p}}=6$, and the solid line connects plus signs that pertain to $N_{\mathrm{p}}=8$.

First, have a look at Fig. 5.6 where zero velocity bandwidth is considered. As expected, the estimator demonstrates an improvement as more pulses become available for processing and as the depth window becomes longer. For increasing $N_{\mathrm{p}}$ and $M$ the estimated mean approaches the true value $\left(0.1 \lambda / T_{\text {prf }}\right)$ only for low velocities, (a). For high velocities, (b), the estimated mean value stays below the true value $\left(0.25 \mathrm{\lambda} / T_{\mathrm{prf}}\right)$ even when high values of $N_{\mathrm{p}}$ and $M$ have been selected. Considering the standard deviations in Fig. 5.6 (c) the decaying tendency is more striking with respect to the raised number of observations than with respect to a longer depth window. For equivalent conditions the observed standard deviations are substantially higher for the higher scaled velocities (Fig. 5.6 (d)) than for the relatively low velocities (Fig. $5.6(\mathrm{c})$ ).

At low velocities (Fig. 5.6 (a), (c)) we see that for signal with the quality factor $Q_{\mathrm{rf}}$ of 2 , the sizes of the depth window $N_{\mathrm{p}}=2$ and 4 do not provide estimates with sufficient accuracy even if the time window $M$ is increased. The improvement is obtained by applying a longer depth window, $N_{\mathrm{p}}$ of at least 6 and a longer time window, $M$ of at least 16 . The limited amount of data for calculation of the likelihood. function (correlation) restricts the performance. At higher velocities, the increase 


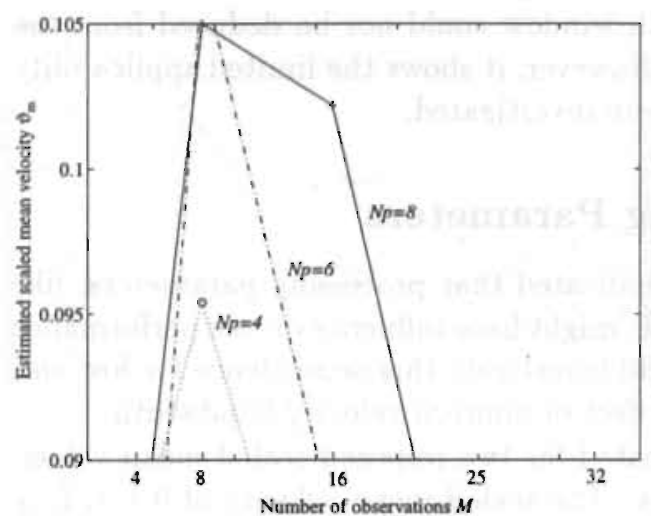

(a)

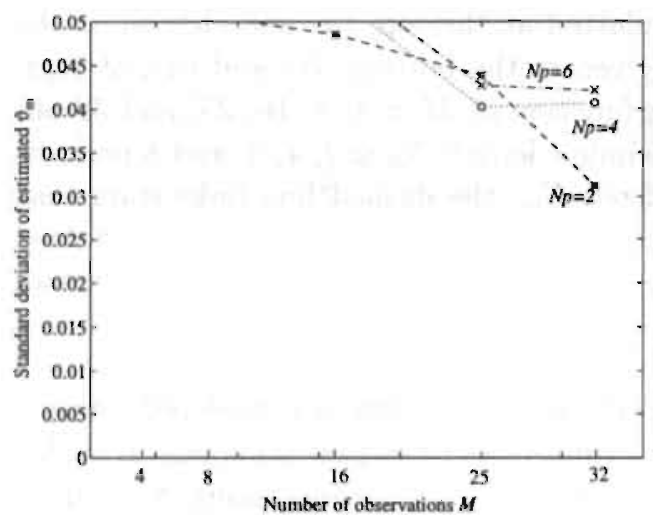

(c)

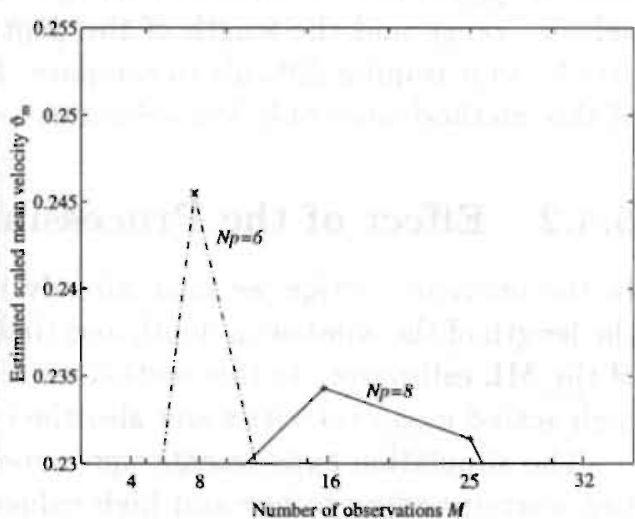

(b)

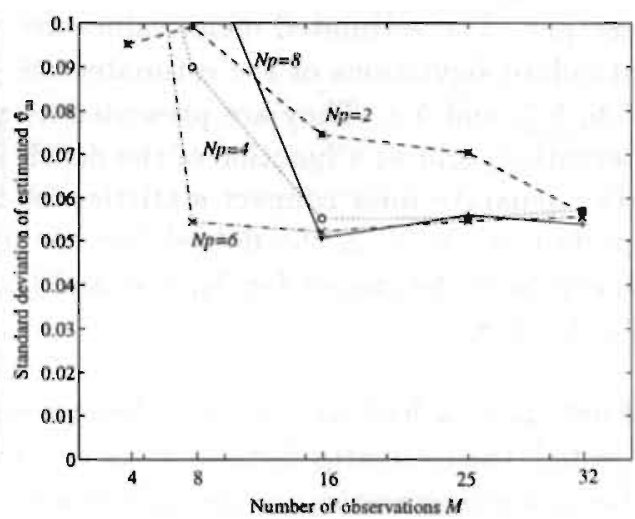

(d)

FIGURE 5.8. Effect of the velocity spread, $\vartheta_{\mathrm{s}}=0.2 \lambda / T_{\mathrm{prf}}$. Four different lines represent four various sizes of the depth window: the dashed line with stars pertains to $N_{\mathrm{p}}$ $=2$, the dotted line with circles to $N_{\mathrm{p}}=4$, the dashed-dotted line with crosses to $N_{\mathrm{p}}=6$, and the solid with plus signs to $N_{\mathrm{p}}=8$. The estimated scaled mean velocity $\hat{\vartheta}_{\mathrm{m}}$ and their standard deviations are plotted for two imposed scaled mean velocities: (a), (c) $\vartheta_{\mathrm{m}}=0.1 \lambda / T_{\mathrm{prf}}$, (b), (d) $\vartheta_{\mathrm{m}}=0.25 \lambda / T_{\mathrm{prf}}$ as function of the time window size of $M=4,8,16,25$, and 32 observations. The other model parameters are not modified: $Q_{\mathrm{rf}}=2, \mathrm{SNR}=10 \mathrm{~dB}$. 
in lengths of the depth and time windows improves the quality measures but still stays behind the values obtained for low velocities under the identical situation. The reasons were already indicated in the previous section and involve the slowly fluctuating point target assumption and dependance of the method on the boundary values of the velocity range considered.

Next, we will inspect the influence of the imposed velocity bandwidth under identical conditions as in the previous figure. Two different values were simulated: $\vartheta_{\mathrm{s}}=$ $0.1 \lambda / T_{\mathrm{prf}}$, and $0.2 \lambda / T_{\mathrm{prf}}$. The resulted estimates and standard deviations are demonstrated in Figs. 5.7, and 5.8. The respective parts of these figures can be compared with each other as well as with Fig. 5.6, where zero velocity bandwidth was considered. It is worth noticing that the selected picture size had to be enlarged in Figs. 5.7 (b), 5.8 (b), and 5.8 (d).

For both low and high mean velocities the performance of the estimator deteriorates strongly with the introduction of a nonzero velocity bandwidth. The difference between estimated and imposed mean scaled velocities (bias) has been increased in comparison to the situation with zero velocity spread. We see that the ML estimator is biased under all conditions considered (Figs. 5.7 (a), (b), 5.8 (a), (b)). Furthermore, the standard deviations are very sensitive to increased velocity bandwidth (Figs. 5.7 (c), (d), 5.8 (c), (d)). The results show that the depth window size has a marginal effect on the performance of the ML estimator in this situation. Thus, it appears that for this estimator the benefits of using a longer depth window are cancelled out by the precision deterioration due to an increased velocity spread. That this method fails under nonzero velocity bandwidth is not surprising because it has no "built in" mechanism to cope with the velocity spread. The fundamental assumption of this method about a point target slowly fluctuating at a constant velocity is violated in the sense that the applied signals simulate signals originating from a large number of scatterers moving with different velocities.

The results of this section show the limited applicability of this method. ML estimates seriously degrade as nonzero velocity bandwidth is introduced. When zero velocity bandwidth is considered, the results indicate a high penalty associated with the use of a very short depth window $\left(N_{p}=2,4\right)$ and a very short time window ( $M=4,8$ observations). However, the performance can be improved by employing more pulses in an estimate and/or by elongation of the depth window. It should be remembered that small values of $N_{\mathrm{p}}$, and $M$ are recommendable for a good spatial and temporal resolutions.

\subsubsection{Effect of the System Properties}

In this section the influence of system parameters such as signal-to-noise ratio (SNR) and quality factor $\left(Q_{\mathrm{rf}}\right)$ will be addressed. First, the effect of SNR on the performance of the ML estimator will be determined. Then the statistics for various quality factors will be compared. 


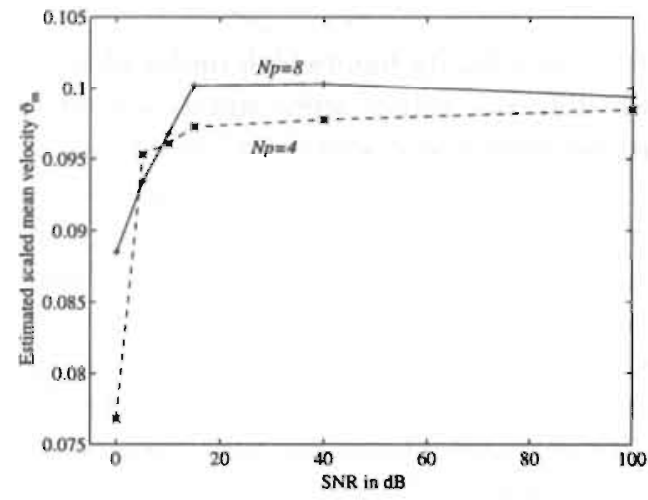

(a)

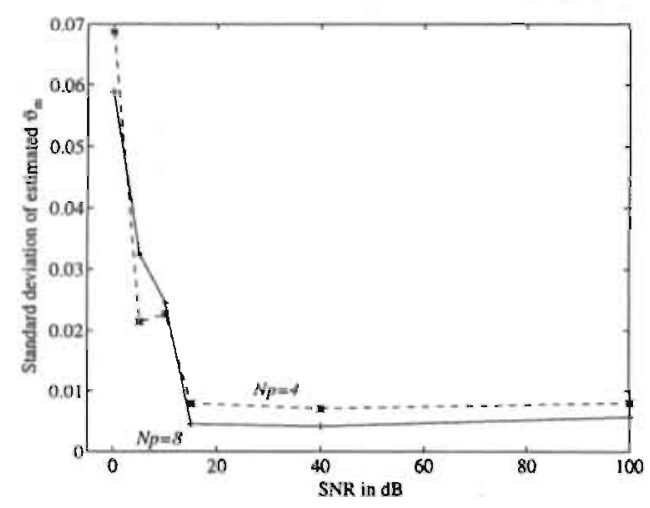

(c)

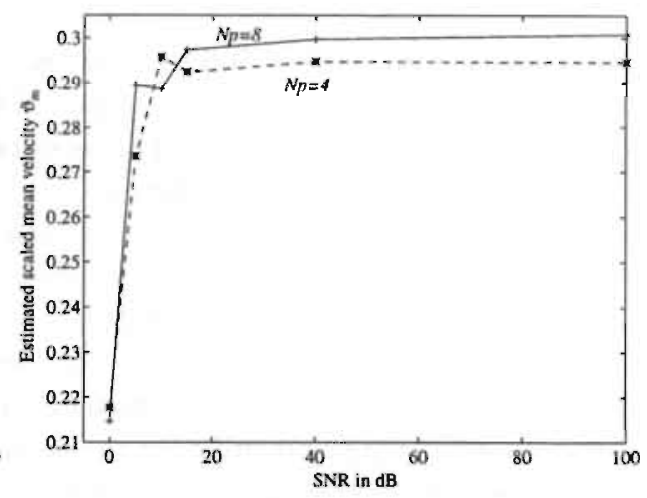

(b)

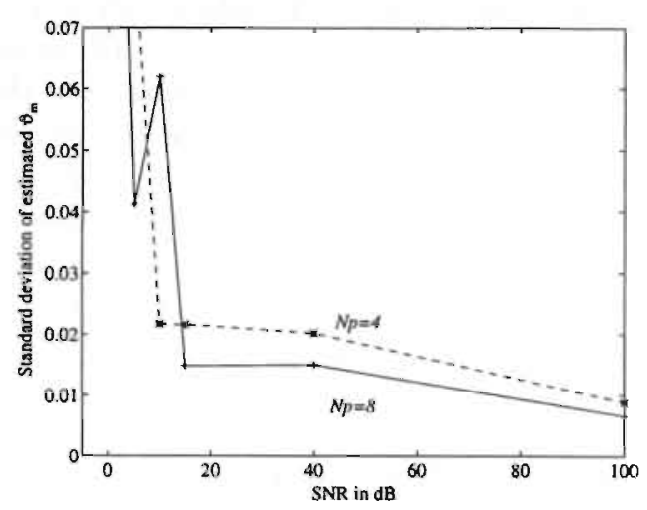

(d)

FIGURE 5.9. Effect of signal to noise ratio. Two different lines represent two various sizes of depth window: the dashed line with stars pertains to $N_{\mathrm{p}}=4$, and the solid with plus signs to $N_{\mathrm{p}}=8$. The estimated scaled mean velocity $\hat{\vartheta}_{\mathrm{m}}$ and their standard deviations are plotted for two imposed scaled mean velocities: (a), (c) $\vartheta_{\mathrm{m}}=0.1 \lambda / T_{\mathrm{prf}}$, (b), (d) $\vartheta_{\mathrm{m}}=0.3 \lambda / T_{\mathrm{prf}}$ as function of signal to noise ratio, $\mathrm{SNR}=0,5,10,15,40,100 \mathrm{~dB}$. The other model parameters are kept constant: $\vartheta_{\mathrm{S}}$ $=0, Q_{\mathrm{rf}}=4, M=25$ observations. 
The estimated scaled mean velocities $\hat{\vartheta}_{\mathrm{m}}$ and their standard deviations can be found for two imposed scaled mean velocities as a function of signal to noise ratio in Fig. 5.9. The SNR varies from $0,5,10,15,40$, to $100 \mathrm{~dB}$, the imposed scaled mean velocity equals for (a), (c) $\vartheta_{\mathrm{m}}=0.1 \lambda / T_{\mathrm{prf}}$, and for (b), (d) $\vartheta_{\mathrm{m}}=0.3 \lambda / T_{\mathrm{prf}}$. Velocity bandwidth, quality factor and time window are fixed to the following values: $\vartheta_{\mathrm{s}}=$ $0, Q_{\mathrm{rf}}=4, M=25$. The development of quality measures (mean and standard deviation) is illustrated for two lengths of depth window, $N_{\mathrm{p}}=4$ in the dashed line with stars, and 8 periods in the solid line with plus signs.

It can be recognized that with higher SNR and longer depth window $N_{\mathrm{p}}$, the estimated mean approaches closer to the true value: $0.1 \lambda / T_{\text {prf }}$ in Fig. 5.9 (a), or $0.3 \lambda / T_{\text {prf }}$ in Fig. 5.9 (c). The relationship between standard deviation and SNR is depicted in Figs. 5.9 (c), and (d). Standard deviations at high velocity (Fig. 5.9 (d)) are two times larger than those for low velocity (Fig. 5.9 (c)). This could be expected. From our exposition in the previous sections we know that the method is limited at high velocities. The results indicate that for poor SNR a small improvement in SNR can dramatically reduce the standard deviation. A change in SNR above a threshold of $15 \mathrm{~dB}$ has a limited effect upon the bias and standard deviation. We also see that results can be made better by extending the size of depth and time window, which has already been discussed in Section 5.4.2 for a SNR of $10 \mathrm{~dB}$.

It can be concluded that for SNRs above $10 \mathrm{~dB}$ the velocity bandwidth will be the greatest source of error because a change in SNR (above $15 \mathrm{~dB}$ ) has little effect on the bias and standard deviation. On the other hand, a drop in SNR from $10 \mathrm{~dB}$ to $0 \mathrm{~dB}$ dramatically affects the quality measures which become very poor even in the absence of velocity spread. In the situation with high SNR the probability that we choose a correct peak is much higher than with low SNR. There will be an error if the noise contributions are large enough to move the peak of the likelihood function away from the correct value. The error is attributed to the fact that the likelihood function was derived under the slowly fluctuating point target assumption and in the simulated situation we consider much more complicated backscattering mechanism. Then the reference signal that enters the likelihood function mismatches the signal actually applied. At poor signal-to-noise ratios the noise contribution increases further this mismatch.

The effect of the quality factor on the performance of the ML estimator was investigated for a SNR of $10 \mathrm{~dB}$ (typical in blood flow measurements). We have included also statistics of the filtered versions (7-th order median filter) of original estimates in order to emphasize the relationship of statistics with respect to the $Q_{\mathrm{rf}}$. As we already know, they can show large variability for low SNRs.

The estimated scaled mean velocities $\hat{\vartheta}_{\mathrm{m}}$ and their standard deviations are displayed in Fig. 5.10 for two imposed scaled mean velocities: (a), (c) $\vartheta_{\mathrm{m}}=0.1 \lambda / T_{\mathrm{pr}}$, and (b), (d) $\vartheta_{\mathrm{m}}=0.25 \lambda / T_{\mathrm{prf}}$ as a function of quality factor $Q_{\mathrm{rf}}=2,4,6,8$. In this figure, the symbol $Q_{\mathrm{s}}$ means the same as $Q_{\mathrm{rf}}$ used in the text. Unfiltered estimates are shown in the dashed-dotted line with crosses for $N_{\mathrm{p}}=Q_{\mathrm{rf}}$, and the solid line 


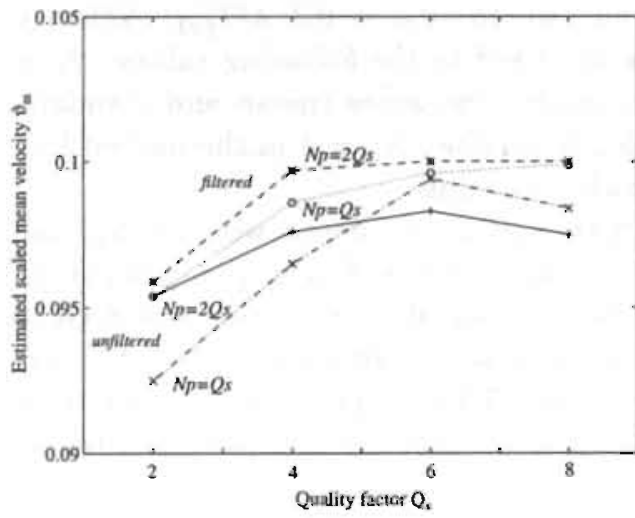

(a)

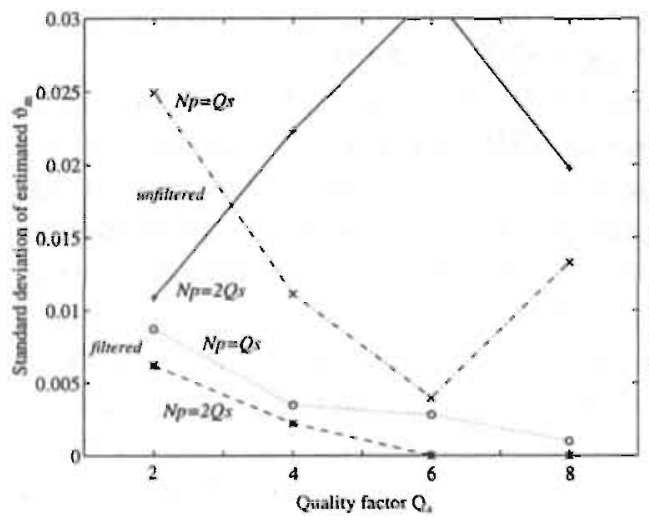

(c)

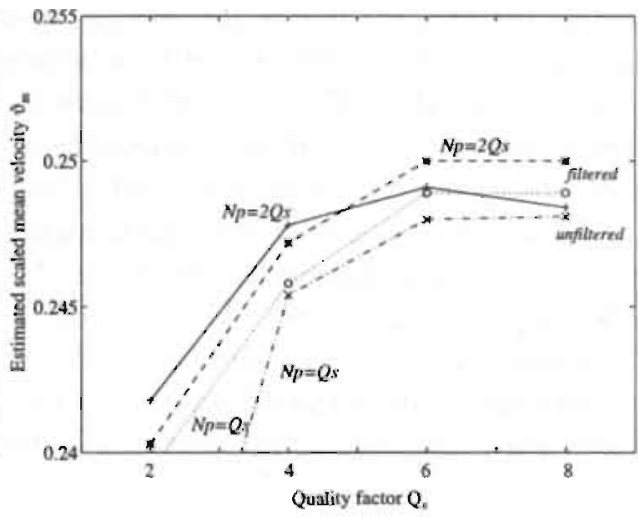

(b)

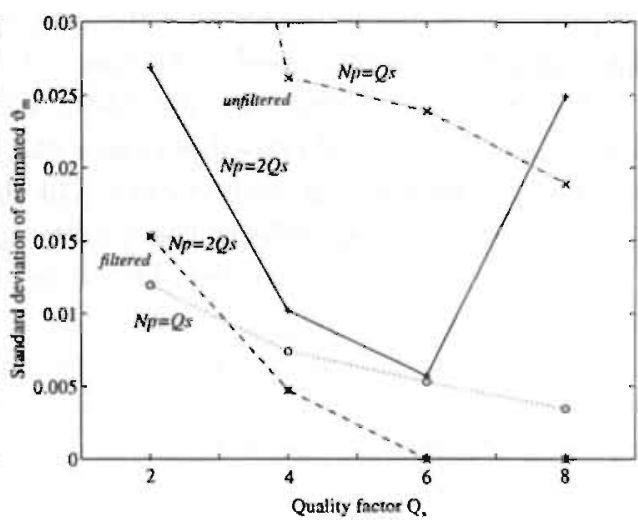

(d)

FIGURE 5.10. Effect of quality factor. In this figure, the symbol $Q_{\mathbb{S}}$ means the same as $Q_{\mathrm{rf}}$ used in the text. The different lines represent two various sizes of the depth window for unfiltered and filtered estimates: the dashed line with stars pertains to filtered version with $N_{\mathrm{P}}=2 Q_{\mathrm{rf}}$, the dotted line with circles to filtered version with $N_{\mathrm{p}}=Q_{\mathrm{rf}}$, the dashed-dotted line with crosses to unfiltered version with $N_{\mathrm{p}}=Q_{\mathrm{rf}}$, and the solid with plus signs to unfiltered version with $N_{\mathrm{p}}=2 Q_{\mathrm{rf}}$. The estimated scaled mean velocities $\hat{\vartheta}_{\mathrm{m}}$ and standard deviations are plotted for two imposed scaled mean velocities: (a), (c) $\vartheta_{\mathrm{m}}=0.1 \lambda / T_{\text {prf }}$, (b), (d) $\vartheta_{\mathrm{m}}=0.25 \lambda / T_{\text {prf }}$ as function of quality factor $Q_{\mathrm{rf}}=2,4,6,8$. The other model parameters are kept constant: $M=25$ observations, $\vartheta_{\mathrm{s}}=0, \mathrm{SNR}=10 \mathrm{~dB}$. 
with plus signs for $N_{\mathrm{p}}=2 Q_{\mathrm{rf}}$. The mean and standard deviations for the filtered version of the estimates are drawn in the dotted line with circles for $N_{\mathrm{p}}=Q_{\mathrm{rf}}$, and in the dashed line with stars for $N_{\mathrm{p}}=2 Q_{\mathrm{rf}}$. The other settings are identical to those in Fig. 5.9.

Since in Section 2.3.1 it was shown that the quality factor determines the number of periods of the impulse response, our choice for the size of depth window, $N_{\mathrm{p}}$, is expressed as integer multiple of $Q_{\mathrm{rf}}$. When we keep the ratio $N_{\mathrm{p}} / Q_{\mathrm{rf}}$ constant, and increase $Q_{\mathrm{rf}}$ (corresponding to elongation of the depth window as well as reduction of spatial resolution), the performance gets better (see Fig. 5.10). The estimated mean approaches the true value, which is $0.1 \lambda / T_{\text {prf }}$ in Fig. 5.10 (a), or $0.25 \lambda / T_{\text {prf }}$ in Fig. 5.10 (c).

The discrepancies in standard deviations of filtered and unfiltered cases are related to the effect of pulse length on jitter magnitude that were studied in [67], [68] for estimators based on the peak detection of the correlation function. Jitter is a second type of error that can be detected when outliers are removed through median filtering. It was indicated [67] that a longer pulse length has the potential to reduce jitter magnitude since it causes an increase in signal power. Increasing signal power reduces the effect of noise and reduces the jitter magnitude. Longer pulses, however, have a flatter likelihood function, which means high subsidiary peaks relative to the primary peak, causing an increase in the number of false peak errors. Hence, when these outliers can be removed long, narrow-band pulses can be used to reduce jitter. The observed zero standard deviations for the filtered case in Figs. 5.10 (c), (d) sub. stantiate this fact. It should be kept in mind, that a longer pulse reduces the spatial resolution.

\subsubsection{Summary}

From the results presented in Sections 5.4.1, 5.4.2, and 5.4.3, we can make the following summary concerning the maximum likelihood estimator.

- The results from our computer simulations indicate an acceptable performance of the maximum likelihood method only for a limited range of signal and processing parameters (high signal-to-noise ratio, long size of depth window, large number of RF returns per estimate, absence of velocity dispersion). The bias and standard deviations as quality measures can be improved to some extent by median filtering. The limited applicability of the ML method is not surprising in this case. We have applied signals whose characteristics change more due to the scattering mechanism than change the characteristics of the transmitted signal due to the "slowly fluctuating point target" assumption. The incompatibility between the assumption and actual characteristics of the applied signal is a reason of misplacement of the peak of the likelihood function that lead to large identification errors. The performance of the method degrades with more complications (scattering, low signal-to-noise ratio, velocity spread within the sample volume, high mean velocity) and is also influenced by the small amount 
of data (a small size of depth and time windows).

- We can compare our observations with those presented in [19], and [21] only on the general level. The characteristics of the signals used in analysis differ. In [21] results obtained from experiments modelling the parabolic flow within a tube are reported. Only limited range of velocities is considered (between 0 and $0.2 \lambda / T_{\text {prf }}$ ) and velocity spread within a sample volume absent. In [19] some theoretical predictions about the form of likelihood function are analyzed. Obviously, the situation changes when the applied signal is more complicated than that assumed by the "slowly fluctuating point target" assumption.

In [21] it was shown for signals with the quality factor $Q_{\mathrm{rf}}$ of 2 , in the absence of noise that the width of the main peak of the the likelihood function becomes smaller as more observations are used to calculate the likelihood function. We obtained similar conclusion since the accuracy of the estimator has been shown to improve with the larger size of temporal window $(M)$. More comparisons are hindered by the differences mentioned above.

An additional discussion of this technique in relation with another velocity estimation method follows in Chapter 7. 


\section{Chapter 6}

\section{Cross-Correlation Model Method}

\subsection{Introduction}

The method that will be presented in this chapter falls under the processing of high frequency signals within our classification scheme. In Section 3.5 we have proposed a classification scheme based on the type of input data, e.g. low frequency or high frequency signals. In the case of processing low frequency signals, the RF signals are transformed to low frequency with respect to a reference frequency. On the other hand, processing of high frequency signals does not involve a signal transformation with respect to a reference frequency. It means that this chapter is dedicated to a technique that does not involve a signal transformation (this transformation was described in Section 3.4). This is an advantage because it avoids an assumption about the carrier frequency of RF returns which can change too much due to the frequency dependent attenuation, introducing a relative error in the estimate.

A brief reference to the reason for our interest in this method has already been given in Section 3.5.2. There it was mentioned that the greatest similarity over successive RF returns can be addressed quantitatively via the correlation function. The following reasoning can be given. Due to the changing distance between transducer and groups of moving scatterers, the RF returns exhibit a gradual translation over a number of pulse returns. The cross-correlation function $\gamma(\Delta x)$ over successive RF returns has therefore certain characteristic features, expressed by equation (3.5.5). From the observed data an estimate of $\gamma(\Delta x)$ can be obtained according to (3.3.5). A search for the depth shift for which the backscattered signals are best aligned is accomplished by seeking the shift which gives a maximum correlation. Obviously, the position of the peak of the correlation function would provide an estimate of the mean distance travelled by the scatterers within the pulse repetition interval $T_{\text {prf }}$ and hence an estimate for the scatterers' velocity. The aim is to locate the peak of the cross-correlation function from discrete data. The precision depends very much on the depth sampling frequency. The higher the sampling frequency, the better the peak of the cross-correlation can be identified. Performing cross-correlation of the signals sampled at high frequency however, demands higher computational efforts. Some modifications of the peak-seeking procedure mentioned in Section 3.5 .2 reduce computational effort but require more sophisticated hardware. 
Suppose, however, that we know the functional form of the cross-correlation $\gamma(\Delta x)$. If we obtain an expression for velocity analytically, we can skip the peakseeking procedure. The estimate of the correlation function is then fitted to the model, yielding a velocity estimate. Because this technique is based on the explicit model of the correlation, respective cross-correlation function, the name CrossCorrelation Model (CCM) was given to it. Obviously, the model of the correlation function should be formed first and a solution for its variable, velocity, should be found. The CCM concept was initiated by de Jong et al. [15] for tissue deformation and by Hoeks et al. [29] for blood velocity estimation. It circumvents the computational demands associated with efforts to alocate the peak of the estimated cross-correlation function. This method has already been successfully applied for the assessment of wall shear rate and arterial impedance in [11].

In the previous studies [15], [29], the CCM method was developed under a narrowband assumption $\left(Q_{\mathrm{rf}} \geq 6\right)$. Our contribution includes an extension of this principle to the wideband signals.

In the subsequent development of this method for blood velocity estimation we begin with a description of the model of the cross-correlation function in Section 6.2. Then we present a solution for the velocity estimate for both narrowband and wideband signals in Section 6.3. In section 6.4 we examine a sample distribution of resulting estimates. Then in Section 6.5 we analyze the performance of the proposed estimator with respect to the processing and system parameters.

\subsection{Model of the Cross-Correlation Function}

The name of the method studied in this chapter indicates that it is based on a model of the cross-correlation function. Our starting point is the two-dimensional (2-D) correlation which follows from (3.5.5) as

$$
\gamma\left(\Delta x, k T_{\mathrm{prf}}\right)=\gamma_{0}\left(\Delta x+v \cos \theta k T_{\mathrm{prf}}\right)
$$

with $\gamma_{0}(\cdot)$ a $1-D$ auto-correlation function. The other symbols in (6.2.1) are broadly used throughout this thesis, for instance they can be found in Section 3.5.1. For simplicity, we will drop the term $\cos \theta$ from the expressions from now on, thereby assuming that only the axial velocity needs to be measured. The final expressions for the estimated mean velocity should be thus divided by $\cos \theta$.

Expression (6.2.1) represents an ideal situation in the absence of measurement noise. In the presence of noise, the expression should be slightly modified because noise contributions are independent in time. This fact causes that the intensities of auto-correlation and cross-correlation differ. Therefore, we introduce a scaling factor $\rho$ to distinguish between auto- and cross-correlation and rewrite (6.2.1) as

$$
\gamma\left(\Delta x, k T_{\mathrm{prf}}\right)=\gamma_{1}\left(\Delta x+v k T_{\mathrm{prf}}\right)=\rho \gamma_{0}\left(\Delta x+v k T_{\mathrm{prf}}\right),
$$


where $\gamma_{1}(\cdot)$ is the 1-D cross-correlation function. The exact value of $\rho$ is not of importance when estimating velocities, what interest us is a model of the crosscorrelation function. We should be able to calculate the specific form of the autocorrelation function $\gamma_{0}(x)$ (and so the cross-correlation function) from the assumed power spectral density $G_{0}(\xi)$ since a power spectrum and correlation function form Fourier pairs.

The CCM concept is based on the assumption that the RF signal has a bandlimited symmetric spectrum concentrated around the center frequency $\xi_{\mathrm{rf}}$. The most straightforward approximation of a power spectrum would be a Gaussian curve. This is very likely if the transmitted signal is considered to have a Gaussian envelope, resulting in a spectrum

$$
G_{0}(\xi)=\frac{1}{2}\left[\left|S\left(\xi-\xi_{\mathrm{rf}}\right)\right|^{2}+\left|S\left(\xi+\xi_{\mathrm{rf}}\right)\right|^{2}\right], \quad \xi \in \mathbf{R},
$$

where $|S(\xi)|^{2}=\exp \left(-\xi^{2} / 2 \xi_{\mathrm{b}}^{2}\right)$ with $\xi_{b}$ the bandwidth frequency. Taking the inverse Fourier transform of $G_{0}(\xi)$ results in a model for the auto-correlation function $\gamma_{0}(x)$ in the form of an exponentially damped sinusoid, mathematically given as

$$
\gamma_{0}(x)=x_{0} \cos \left(2 \pi \xi_{\mathrm{rf}} x\right) \exp \left(-2 \pi^{2} \xi_{\mathrm{b}}^{2} x^{2}\right)
$$

where $x_{0}$ is a constant that includes signal and noise powers. Substitution of (6.2.1) into (6.2.2) gives the model of the cross-correlation function.

\subsection{Cross-Correlation Model Estimator of Blood Velocity}

To dcrive an expression for the velocity estimate from the correlation function we make use of $(6.2 .2)$ and recognize that for $k=0$ we are dealing with the autocorrelation function $\gamma_{0}(x)$. A general model of correlation over RF signals can be stated as

$$
\gamma\left(\Delta x, k T_{\mathrm{pr}}\right)= \begin{cases}\rho \gamma_{0}\left(\Delta x+v_{\mathrm{m}} k T_{\mathrm{prf}}\right), & \text { if } k \neq 0, \\ \gamma_{0}(\Delta x), & \text { if } k=0,\end{cases}
$$

where $\gamma_{0}(x)$ is given by (6.2.4), and $v_{\mathrm{m}}$ is the mean velocity at which the scatterers have moved within $k T_{\text {prf }}$. In the sequel we wish to work with normalized quantities with respect to the spatial sampling frequency, $\xi_{\mathrm{s}}$, and temporal sampling frequency, $f_{\text {prf }}$. The connecting considerations were already mentioned in Section 3.2. There, the normalized frequencies $\Psi$ and $F$ were introduced as ratios: $\Psi=\xi / \xi_{\mathrm{s}}$, and $F=f / f_{\text {prf }}$. The dimensionless velocity $\varphi$ is then defined by expression (3.2.3). Using the normalized quantities in the correlation, for instance, $\gamma(i, k)$, reflects correlation $\gamma\left(i x_{\mathrm{s}}, k T_{\mathrm{prf}}\right)$, where sampling distance $x_{\mathrm{s}}$, and period $T_{\mathrm{prf}}$ are the inverses of the sampling frequencies $\xi_{\mathrm{s}}$, and $f_{\mathrm{pr} f}$ respectively.

Having a model of a correlation $\gamma\left(\Delta x, k T_{\text {prf }}\right)$, described by (6.3.1) and (6.2.4), we will in this section present two estimates of the mean velocity. The two cases differ in the assumptions taken. First, it is assumed that the transmitted signal is a narrowband signal (in Section 6.3.1), later this assumption will be dropped (in Section 6.3.2). 


\subsubsection{Narrowband CCM Velocity Estimator}

The derivation of the CCM velocity estimate for narrowband signals is given in [14] for the correlation function corresponding to the rectangular power spectral density. An analogical derivation for narrowband signals but for a Gaussian power spectral shape is formulated in [29]. It is worth mentioning that both final expressions for the velocity estimate, starting from different shapes of power spectrum, are equivalent for the case of narrowband signals.

The narrowband assumption simplifies the correlation function, since as bandwidth $\xi_{b}$ approaches zero, the exponential term of (6.2.4) will approach one. Therefore, the model of the auto-correlation function (6.2.4) is rewritten as

$$
\gamma(i, k)= \begin{cases}\rho x_{0} \cos \left(2 \pi \Psi_{\mathrm{rf}}\left(i+\varphi_{\mathrm{m}} k\right)\right), & \text { if } k \neq 0 \\ x_{0} \cos \left(2 \pi \Psi_{\mathrm{rf}} i\right), & \text { if } k=0 .\end{cases}
$$

We will not repeat all the derivation steps here, rather the final result will be stated in order to allow for a comparison with a new estimator that will be presented in the next section. The correlations at the following five different lags

$$
\begin{aligned}
\gamma(0,0) & =x_{0} \\
\gamma(1,0) & =x_{0} \cos \left(2 \pi \Psi_{\mathrm{rf}}\right), \\
\gamma(0,1) & =\rho x_{0} \cos \left(2 \pi \Psi_{\mathrm{rf}} \varphi_{\mathrm{m}}\right), \\
\gamma(1,1) & =\rho x_{0} \cos \left(2 \pi \Psi_{\mathrm{rf}}\left(1+\hat{\varphi}_{\mathrm{m}}\right)\right) . \\
\gamma(-1,1) & =\rho x_{0} \cos \left(2 \pi \Psi_{\mathrm{rf}}\left(-1+\varphi_{\mathrm{m}}\right)\right),
\end{aligned}
$$

are sufficient to solve for the dimensionless mean velocity $\varphi_{\mathrm{m}}$. Let

$$
\begin{aligned}
& \alpha=\arccos \left(\frac{\hat{\gamma}(1,0)}{\hat{\gamma}(0,0)}\right), \\
& \beta=\arctan 2\left(\frac{\hat{\gamma}(1,1)-\hat{\gamma}(-1,1)}{2}, \hat{\gamma}(0,1) \sin (\alpha)\right),
\end{aligned}
$$

where $\hat{\gamma}(n, m)$ is an estimator of $\gamma(n, m)$ given by equation (3.3.5). Then the estimate of dimensionless mean velocity $\hat{\varphi}_{\mathrm{m}}$ equals

$$
\hat{\varphi}_{\mathrm{m}}=\frac{\beta}{\alpha} .
$$

The estimate of scaled mean velocity, $\hat{\vartheta}_{\mathrm{m}}$, that is used for simulations, can be obtained by applying (4.3.2). Therefore,

$$
\hat{\vartheta}_{\mathrm{m}}=\frac{\hat{\varphi}_{\mathrm{m}}}{R_{\mathrm{s}}}
$$

Equation (3.2.4), or (4.3.1) will give the estimated mean velocity in $\mathrm{ms}^{-1}$ as

$$
\hat{v}_{\mathrm{m}}=\frac{\beta}{\alpha} \frac{T_{\text {prf }}}{f_{\mathrm{s}}} \frac{c}{2}
$$


with $c$ the acoustic velocity. Also other unknown parameters of the correlation function, $x_{0}, \rho$, and $\Psi_{\mathrm{rf}}$ can be solved from the above (6.3.3) correlations. Note, that $T_{\text {prf }}, c$, and $f_{\mathrm{s}}$ are a priori known quantities. Evaluating $\alpha$ of (6.3.4) will result in $2 \pi \Psi_{\mathrm{rf}}$, from which the estimate of the spatial carrier frequency $\hat{\xi}_{\mathrm{rf}}$ follows as

$$
\hat{\xi}_{\mathrm{rf}}=\frac{\alpha}{2 \pi} \xi_{\mathrm{s}} .
$$

This fact is an advantage for the processing of high-frequency signals with respect to the processing of low-frequency signals in which the spatial carrier frequency is assumed to be known and equal to the emitted frequency.

\subsubsection{Wideband CCM Velocity Estimator}

As was already indicated, we will now drop our assumption about narrowband signals $\left(Q_{\mathrm{rf}}>6\right)$. The CCM concept will be extended also to signals with quality factor $Q_{\mathrm{rf}}>1$, wideband signals. The bandwidth $\xi_{\mathrm{b}}$ cannot be neglected in (6.2.4), thus the auto-correlation model $\gamma_{0}(x)$ is given by (6.2.4). The general correlation model of (6.3.1) is rewritten in normalized quantities as follows

$$
\gamma(i, k)= \begin{cases}\rho x_{0} \cos \left(2 \pi \Psi_{\mathrm{rf}}\left(i+\varphi_{\mathrm{m}} k\right) \exp \left(-2 \pi^{2} \Psi_{\mathrm{b}}^{2}\left(i+\varphi_{\mathrm{m}} k\right)^{2}\right),\right. & \text { if } k \neq 0, \\ x_{0} \cos \left(2 \pi \Psi_{\mathrm{rf}} i\right) \exp \left(-2 \pi^{2} \Psi_{\mathrm{b}}^{2} i^{2}\right), & \text { if } k=0 .\end{cases}
$$

In order to solve for the normalized mean velocity $\varphi_{\mathrm{m}}$, the correlations at the following lags have to be considered:

$$
\begin{aligned}
\gamma(0,0) & =x_{0} \\
\gamma(1,0) & =x_{0} \cos \left(2 \pi \Psi_{\mathrm{rf}}\right) \exp \left(-2 \pi^{2} \Psi_{\mathrm{b}}^{2}\right), \\
\gamma(2,0) & =x_{0} \cos \left(4 \pi \Psi_{\mathrm{rf}}\right) \exp \left(-8 \pi^{2} \Psi_{\mathrm{b}}^{2}\right), \\
\gamma(0,1) & =\rho x_{0} \cos \left(2 \pi \Psi_{\mathrm{rf}} \varphi_{\mathrm{m}}\right) \exp \left(-2 \pi^{2} \Psi_{\mathrm{b}}^{2} \varphi_{\mathrm{m}}^{2}\right), \\
\gamma(1,1) & =\rho x_{0} \cos \left(2 \pi \Psi_{\mathrm{rf}}\left(1+\varphi_{\mathrm{m}}\right)\right) \exp \left(-2 \pi^{2} \Psi_{\mathrm{b}}^{2}\left(1+\varphi_{\mathrm{m}}\right)^{2}\right), \\
\gamma(-1,1) & =\rho x_{0} \cos \left(2 \pi \Psi_{\mathrm{rf}}\left(-1+\varphi_{\mathrm{m}}\right)\right) \exp \left(-2 \pi^{2} \Psi_{\mathrm{b}}^{2}\left(-1+\varphi_{\mathrm{m}}\right)^{2}\right) .
\end{aligned}
$$

We define the following normalized correlations (normalized with respect to the power, $\gamma(0,0)$, respect. $\gamma(0,1)$, that are different from zero)

$$
\begin{aligned}
A_{1} & =\frac{\gamma(1,0)}{\gamma(0,0)}=\cos \left(2 \pi \Psi_{\mathrm{rf}}\right) \exp \left(-2 \pi^{2} \Psi_{\mathrm{b}}^{2}\right) \\
A_{2} & =\frac{\gamma(2,0)}{\gamma(0,0)}=\cos \left(4 \pi \Psi_{\mathrm{rf}}\right) \exp \left(-8 \pi^{2} \Psi_{\mathrm{b}}^{2}\right) \\
C_{1} & =\frac{\gamma(1,1)}{\gamma(0,1)}=\frac{\cos \left(2 \pi \Psi_{\mathrm{rf}}\left(1+\varphi_{\mathrm{m}}\right)\right)}{\cos \left(2 \pi \Psi_{\mathrm{rf}} \varphi_{\mathrm{m}}\right)} \exp \left(-2 \pi^{2} \Psi_{\mathrm{b}}^{2}\left(1+2 \varphi_{\mathrm{m}}\right)\right. \\
C_{-1} & =\frac{\gamma(-1,1)}{\gamma(0,1)}=\frac{\cos \left(2 \pi \Psi_{\mathrm{rf}}\left(-1+\varphi_{\mathrm{m}}\right)\right)}{\cos \left(2 \pi \Psi_{\mathrm{rf}} \varphi_{\mathrm{m}}\right)} \exp \left(-2 \pi^{2} \Psi_{\mathrm{b}}^{2}\left(1-2 \varphi_{\mathrm{m}}\right)\right.
\end{aligned}
$$


Let us define two variables, $\alpha_{w}$ and $\beta_{w}$, that appear to be useful in the derivations and can be verified using goniometric relations:

$$
\begin{aligned}
\alpha_{w} & =\left(A_{1}^{4}-A_{2}\right)^{\frac{1}{4}}=\left|\sin \left(2 \pi \Psi_{\mathrm{rf}}\right)\right| \exp \left(-2 \pi^{2} \Psi_{\mathrm{b}}^{2}\right), \\
\boldsymbol{\beta}_{w} & =\left(A_{1}^{2}-C_{1} C_{-1}\right)^{\frac{1}{2}}=\left|\sin \left(2 \pi \Psi_{\mathrm{rf}}\right)\right|\left|\tan \left(2 \pi \Psi_{\mathrm{rf}} \varphi_{\mathrm{m}}\right)\right| \exp \left(-2 \pi^{2} \Psi_{\mathrm{b}}^{2}\right) \\
& =\alpha_{w}\left|\tan \left(2 \pi \Psi_{\mathrm{rf}} \varphi_{\mathrm{m}}\right)\right| .
\end{aligned}
$$

We now derive an expression for the normalized spatial carrier frequency $\Psi_{\text {rf }}$. If we divide $\alpha_{w}$ by $A_{1}$, we obtain

$$
\frac{\alpha_{w}}{A_{1}}=\frac{\left|\sin \left(2 \pi \Psi_{\mathrm{rf}}\right)\right|}{\cos \left(2 \pi \Psi_{\mathrm{rf}}\right)}
$$

from which it follows that the estimate of the normalized spatial carrier frequency is

$$
\hat{\Psi}_{\mathrm{rf}}=\frac{\arctan 2\left(\alpha_{w}, A_{1}\right)}{2 \pi} .
$$

To derive an expression for the dimensionless mean velocity $\varphi_{\mathrm{m}}$ we shall make use of the second relation of (6.3.12) and recognize that

$$
\tan \left(2 \pi \Psi_{\mathrm{rf}} \varphi_{\mathrm{m}}\right)=\left|\frac{\beta_{w}}{\alpha_{w}}\right| .
$$

Equivalently,

$$
2 \pi \Psi_{\mathrm{rf}}\left|\varphi_{\mathrm{m}}\right|=\arctan 2\left(\left|\beta_{w}\right|, \operatorname{sign}(\gamma(0,1)) \alpha_{w}\right),
$$

where the function $\operatorname{sign}(\cdot)$ returns 1 if the element is positive, 0 if it equals zero and -1 if it is negative. Using (6.3.14) in (6.3.16) gives

$$
\left|\varphi_{\mathrm{m}}\right|=\frac{\arctan 2\left(\left|\beta_{w}\right|, \operatorname{sign}(\gamma(0,1)) \alpha_{w}\right)}{\arctan 2\left(\alpha_{w}, A_{1}\right)} .
$$

The modification of (6.3.17) to include the sign, which indicates the direction of flow, is obtained from the observation that

$$
\begin{aligned}
\operatorname{sign}(\gamma(-1,1)-\gamma(1,1)) & \cdots a_{0}\left(\left(a_{1}-a_{2}\right) \cos \left(2 \pi \Psi_{\mathrm{rf}}\right) \cos \left(2 \pi \Psi_{\mathrm{rf}} \varphi_{\mathrm{m}}\right)\right. \\
+ & \left.\left(a_{1}+a_{2}\right) \sin \left(2 \pi \Psi_{\mathrm{rf}}\right) \sin \left(2 \pi \Psi_{\mathrm{rf}} \varphi_{\mathrm{III}}\right)\right),
\end{aligned}
$$

where $a_{0}=\rho x_{0} \exp \left(-2 \pi^{2} \Psi_{\mathrm{b}}^{2}\left(1+\varphi_{\mathrm{m}}^{2}\right)\right), a_{1}=\exp \left(4 \pi^{2} \Psi_{\mathrm{b}}^{2} \varphi_{\mathrm{m}}\right), a_{2}=\exp \left(-4 \pi^{2} \Psi_{\mathrm{b}}^{2} \varphi_{\mathrm{m}}\right)$ are always positive. Let us discover for which values of $\varphi_{\mathrm{m}}$ the right-hand side of (6.3.18) gives zero, by solving the following equation

$$
\frac{a_{1}-a_{2}}{a_{1}+a_{2}}=-\frac{\sin \left(2 \pi \Psi_{\mathrm{rf}}\right) \sin \left(2 \pi \Psi_{\mathrm{rf}} \varphi_{\mathrm{m}}\right)}{\cos \left(2 \pi \Psi_{\mathrm{rf}}\right) \cos \left(2 \pi \Psi_{\mathrm{rf}} \varphi_{\mathrm{m}}\right)} .
$$

Equivalently,

$$
\tanh \left(4 \pi^{2} \Psi_{\mathrm{b}}^{2} \varphi_{\mathrm{m}}\right)=-\tan \left(2 \pi \Psi_{\mathrm{rf}}\right) \tan \left(2 \pi \Psi_{\mathrm{rf}} \varphi_{\mathrm{m}}\right)
$$


The hyperbolic tangent function is positive for $\varphi_{\mathrm{m}}>0$, and is negative for $\varphi_{\mathrm{m}}<0$. The right side of equation (6.3.20) is with respect to $\varphi_{\mathrm{m}}$ positive for $\varphi_{\mathrm{m}}<0$, and negative for $\varphi_{\mathrm{m}}>0$. Therefore, there exists only one intersection point of these functions, namely $\varphi_{\mathrm{m}}=0$. Furthermore, when we substitute for $\varphi_{\mathrm{m}}=1$ into (6.3.18), the result of $\operatorname{sign}(\gamma(-1,1)-\gamma(1,1))=1$, and for $\varphi_{\mathrm{m}}=-1, \operatorname{sign}(\gamma(-1,1)-$ $\gamma(1,1))=-1$. Then from the continuity of the function (6.3.18) we conclude that

$$
\operatorname{sign}(\gamma(-1,1)-\gamma(1,1))= \begin{cases}1, & \text { if } \varphi_{\mathrm{m}}>0 \\ 0, & \text { if } \varphi_{\mathrm{m}}=0, \\ -1, & \text { if } \varphi_{\mathrm{m}}<0\end{cases}
$$

From (4.3.2) and our above development it is clear that an estimate of the scaled mean velocity is given as

$$
\hat{\vartheta}_{\mathrm{m}}=\frac{\hat{\varphi}_{\mathrm{m}}}{R_{\mathrm{s}}}=\operatorname{sign}(\hat{\gamma}(-1,1)-\hat{\gamma}(1,1)) \frac{\left|\hat{\varphi}_{\mathrm{m}}\right|}{R_{\mathrm{s}}},
$$

where (6.3.17) has to be substituted for $\left|\hat{\varphi}_{\mathrm{m}}\right|$. All variables with ${ }^{\wedge}$ are obtained from the estimated correlations of (6.3.10) by an estimator (3.3.5). The estimate of mean velocity in $\mathrm{ms}^{-1}$ directly follows from equation (4.3.1) and equals

$$
\hat{v}_{\mathrm{m}}=\operatorname{sign}(\hat{\gamma}(-1,1)-\hat{\gamma}(1,1))\left|\hat{\varphi}_{\mathrm{m}}\right| \frac{T_{\mathrm{prf}}}{f_{\mathrm{s}}} \frac{c}{2}
$$

where $c$ is the acoustic velocity.

\subsection{Statistical Properties of the CCM Estimator}

From our development in Section 6.3.2 it follows that by estimating the correlation function of the RF signal at several lags, the velocity can be deduced and used to characterize the blood flow. Therefore, the statistical properties of the wideband CCM velocity estimator will be affected by the statistical properties of the correlation function estimator (3.3.5).

The statistical properties of the correlation estimates have been studied in some detail in [59] where analytical expressions for the second-order moments can be found. Computer simulations in [60] show that by normalizing the correlation estimates with the signal power estimate, the probability density function fits to a Gaussian distribution. This observation can be a starting point for us since the wideband CCM velocity estimator is based on the normalized correlations (6.3.11). However, the velocity estimator (6.3.22) gives a nonlinear mapping of the normalized correlation estimates, which makes it difficult to deduce analytical expressions for the probability density function. We have chosen to address it by means of a simulation study with a sample set $S$ of 1000 estimates.

A data set was generated according to the simulation model of Chapter 4 with the model settings defined by equation (4.3.3), for $M=25$ observations, $N_{\mathrm{p}}=8$ periods, 


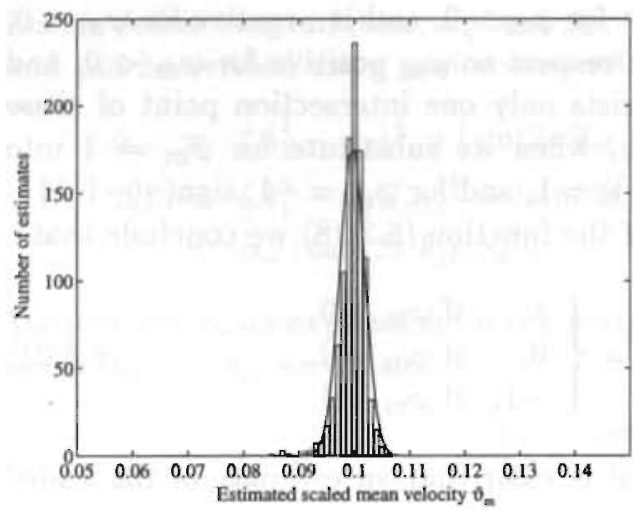

(a)

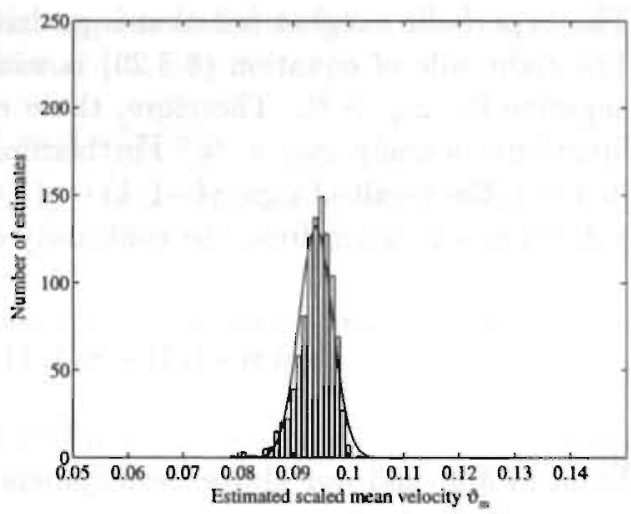

(b)

Figure 6.1. Two histograms of the CCM estimates. (a) shows the wideband CCM estimates, (b) depicts the distribution of the narrowband CCM estimates. The model parameters are kept constant: $M=25$ observations, $N_{\mathrm{p}}=8$ periods, $\vartheta_{\mathrm{m}}=$ $0.1 \lambda / T_{\mathrm{prf}}, \vartheta_{\mathrm{s}}=0, Q_{\mathrm{rf}}=2, \mathrm{SNR}=10 \mathrm{~dB}$.

$\vartheta_{\mathrm{m}}=0.1 \lambda / T_{\mathrm{prf}}, \vartheta_{\mathrm{s}}=0, Q_{\mathrm{rf}}=2, \mathrm{SNR}=10 \mathrm{~dB}$. The other model parameters are fixed at values stated in Section 4.3. Notice, that this setting is identical to the one used in Section 5.3 on statistical properties of the ML estimator. The simulations were run in parallel for the same set of data.

The distribution of CCM velocity estimates is plotted in Fig. 6.1 for (a) wideband (6.3.22) and (b) narrowband (6.3.6) estimates of the imposed scaled mean velocity $\vartheta_{\mathrm{m}}=0.1 \lambda / T_{\text {prf }}$. We observe that histograms of CCM estimates have a form close to the Gaussian distribution. In case of the wideband CCM estimator, Fig. 6.1 (a), the histogram is concentrated at the imposed scaled mean velocity $\left(0.1 \lambda / T_{\mathrm{prf}}\right)$, and is narrow, which means that the estimates are very close to the true value. To their distribution a Gaussian curve is fitted with estimated mean velocity, 0.0996 $\lambda / T_{\mathrm{prf}}$, and standard deviation, $0.0022 \lambda / T_{\mathrm{prf}}$, which is a good approximation to the distribution function of estimates.

The shape of the histogram for the narrowband CCM velocity estimates in Fig. 6.1 (b) obeys the Gaussian curve with mean $0.094 \lambda / T_{\text {prf }}$, and standard deviation $0.0030 \lambda / T_{\text {prf }}$. It can be seen that the estimates are not concentrated around the true value, $0.1 \lambda / T_{\mathrm{prf}}$. This deviation is not surprising. The bias in the narrowband CCM velocity estimator is caused by its assumption on the bandwidth of the signal. The estimator is designed for signals with a quality factor of at least six, while for the experimental situation of Fig. 6.1 the quality factor of 2 was used, which corresponds to wideband signals. The reason we have included it here is to compare the performance of both estimators. It should be clear, that for signals with high quality factor, both estimators behave in a similar way, while for signals with low quality factor, the wideband CCM estimator (6.3.23) is superior. 
The conclusion from this section is that the CCM velocity estimators (narrowband and wideband) converge in distribution to the Gaussian distribution, which means that the first- and second-order moments give an adequate statistical description of the set of estimates.

\subsection{Performance of the Wideband CCM Estimator}

To test the behavior of the new wideband CCM estimator and to investigate its practical potential the computer simulation approach is preferred, since due to the complexity involved the theoretical analysis would be very complicated. The wideband CCM estimator, (6.3.22) is a nonlinear mapping of several correlations. The analytical derivation of standard deviation would require a linearization procedure that may lead to oversimplifications, and the final expression would no longer correctly describe the CCM behavior.

A data set was generated according to the simulation model of Chapter 4 for the settings defined by equation (4.3.3). The defaults are described in Section 4.3. Since the (imposed) properties of the simulated signals are completely known a qualitative assessment of the performance of the CCM estimator is possible. This task is evaluated in terms of the bias and standard deviation of resulting estimates obtained from a sample set $S$ according to equations (3.3.8), and (3.3.7). For computational reasons, we reduced the sample set to $S=100$ estimates in comparison to the sample set used for analysis in the previous section. The robustness properties of the estimator can also be addressed by means of a simulation experiment. Assumptions about correlation model may be deliberately violated to obtain an indication of sensitivity to that violation.

In the following subsections we investigate the effect of numerous different parameters on the performance of the CCM estimator individually. In Section 6.5.1 we study the influence of the scaled mean velocity, in Section 6.5 .2 we inquire into the effects of sizes of the temporal and spatial windows, and into the effect of velocity dispersion. In Section 6.5.3 we pay attention to the effect of the signal-to-noise ratio and quality factor. We then analyze in Section 6.5.4 the robustness of the wideband CCM method with respect to the assumption on the symmetric shape of the spectrum. In the last Section 6.5.5 we summarize the observations obtained from the simulation results.

\subsubsection{Effect of Velocity}

In this section we will inspect the behavior of the wideband CCM estimator as a function of scaled mean velocity, $\vartheta_{\mathrm{m}}$. The difference between imposed and estimated scaled mean velocity, respective bias is depicted as plus signs in Fig. 6.2 for velocities within the range of $(-0.4,0.4) \lambda / T_{\text {prf }}$ with step of $0.02 \lambda / T_{\text {pri }}$. Around these bias 


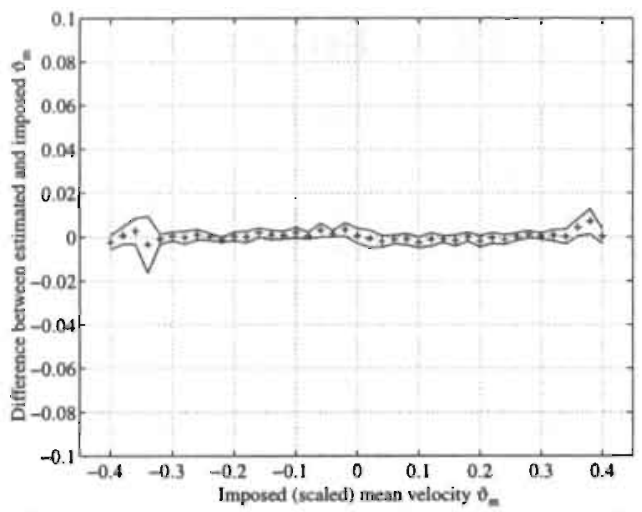

(a)

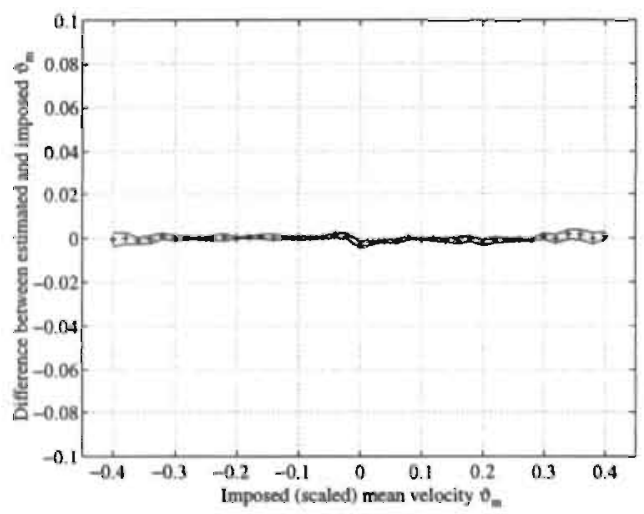

(c)

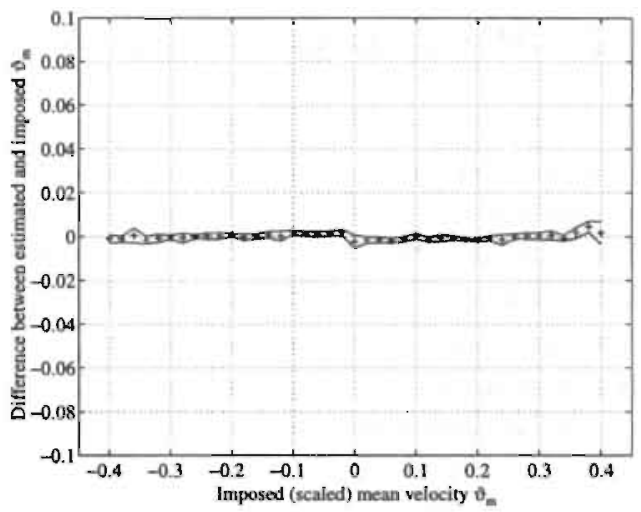

(b)

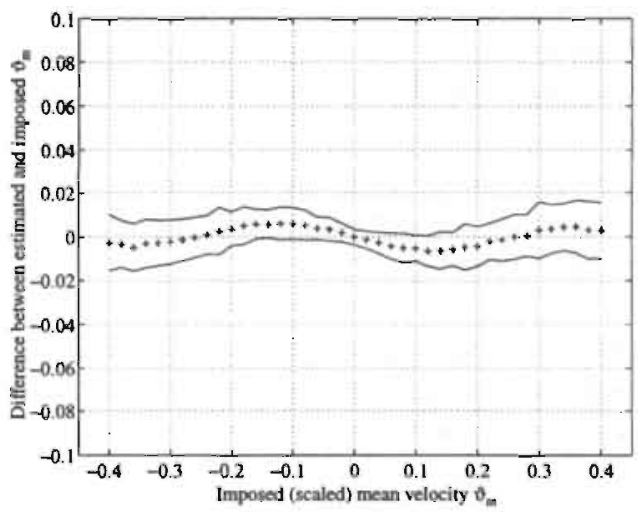

(d)

FigURE 6.2. The difference between estimated and imposed scaled mean velocity, $29 \mathrm{~m}$, with a $95 \%$ confidence interval. (a) $N_{\mathrm{p}}=4$ periods, $M=8$ observations, (b) $N_{\mathrm{p}}$ $=4$ periods, $M=16$ observations, (c) $N_{\mathrm{p}}=8$ periods, $M=16$ observations, (d) $N_{\mathrm{p}}=8$ periods, $M=16$ observations, for the narrowband CCM estimates. The other model parameters are kept constant: $\vartheta_{\mathrm{S}}=0, Q_{\mathrm{rf}}=2, \mathrm{SNR}=10 \mathrm{~dB}$. 
values a $95 \%$ confidence interval (two times the standard deviation) is drawn in order to gain a picture of the velocity resolution. All parts of Fig. 6.2 (a), (b), (c), and (d) are generated with zero velocity spread, quality factor $Q_{\mathrm{rf}}=2$, and $\mathrm{SNR}=10 \mathrm{~dB}$. Window length in depth and in time differ per plot.

The resulting estimates in Fig. 6.2 (a) are obtained for a depth window of 4 periods, and time window of 8 pulses. We observe that the behavior is stable for the entire range of considered velocities. Only small fluctuations are visible at higher absolute velocities. The next plot, Fig. 6.2 (b), illustrates the situation when the number of observations per estimate is doubled $(M=16)$. It causes a reduction in difference between estimated and true velocity as well as a decrease in width of the $95 \%$ confdence interval. Elongating the window in depth, $N_{\mathrm{p}}=8$ periods, as seen in Fig. 6.2 (c), brings further improvement (smaller width of the confidence interval) for most velocities. The observed deviations around zero velocity are not critical, since in blood velocity estimation zero velocities are suppressed by the wall filter.

In Fig. 6.2 (d) the narrowband CCM velocity estimates are evaluated. The same data set was used as for Fig. 6.2 (c). As expected, it is a biased estimator for $Q_{\mathrm{rf}}=$ 2 because its assumption of narrowband signals is not fulfilled.

Figs. 6.2 (a), (b), and (c) promise an excellent velocity resolution and prove a significant improvement in the performance of the wideband CCM velocity estimator in comparison to the narrowband CCM estimator (Fig. 6.2 (d)) in the wideband situation.

\subsubsection{Effect of the Processing Parameters}

From the previous section it may be expected that an increase in the length of the processing parameters, like depth and time window will improve the performance of the wideband CCM estimator. To investigate this hypothesis properly, the simulation experiments were repeated for two specific scaled mean velocities under a velocity bandwidth of $0,0.1 \lambda / T_{\mathrm{prf}}$, and $0.2 \lambda / T_{\mathrm{prf}}$ respectively. The imposed scaled mean velocity of $0.1 \lambda / T_{\text {prf }}$ represents low velocities while $0.25 \lambda / T_{\text {prf }}$ represents high velocities. Remember that the velocity bandwidth of for instance $0.2 \lambda / T_{\mathrm{prf}}$ simulates the velocity field with velocity components within $\pm 0.1 \lambda / T_{\text {prf }}$ of the imposed scaled mean velocity.

The results are documented in Fig. 6.3 for zero velocity spread, in Fig. 6.4 for velocity spread of $0.1 \lambda / T_{\text {prf }}$, and in Fig. 6.5 for velocity spread of $0.2 \lambda / T_{\text {prf }}$. The default values $Q_{\mathrm{rr}}=2$, and $\mathrm{SNR}=10 \mathrm{~dB}$ were chosen for all simulations studied in this section. The estimated mean values are plotted at the top, (a) and (b), with the standard deviations at the bottom, (c) and (d) of Figs. 6.3, 6.4, 6.5. They are presented as a function of $M=4,8,16,25$, and 32 observations and a function of depth window length $N_{\mathrm{p}}=2,4,6$, and 8 periods. Separate lines connect the statistics for fixed $N_{\mathrm{p}}$ : the dashed line links stars that pertain to $N_{\mathrm{p}}=2$, the dotted line connects circles for $N_{\mathrm{p}}=4$, the dashed-dotted line relates the crosses for $N_{\mathrm{p}}=$ 6 , and the solid line connects plus signs that pertain to $N_{\mathrm{p}}=8$. 


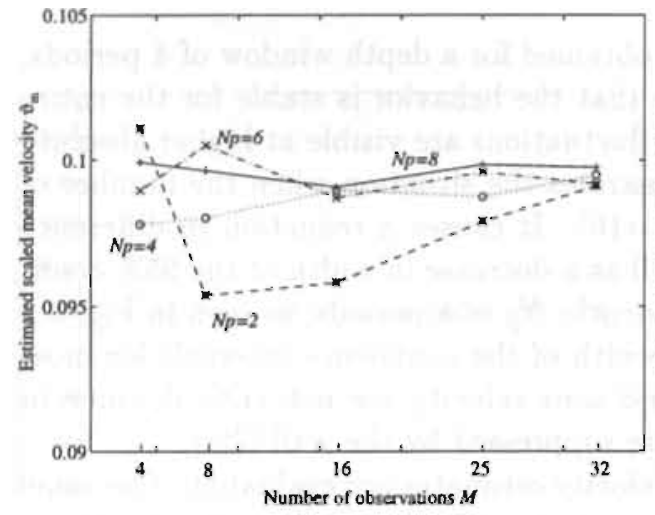

(a)

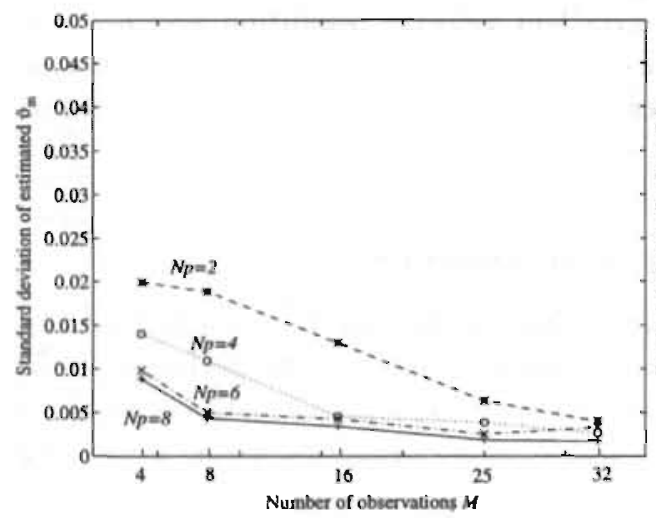

(c)

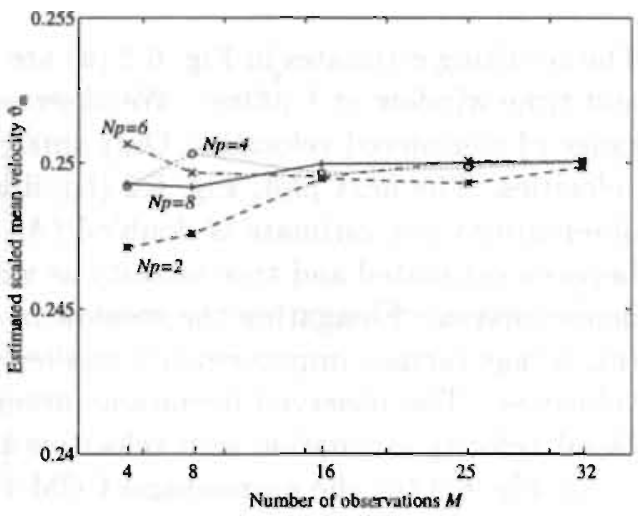

(b)

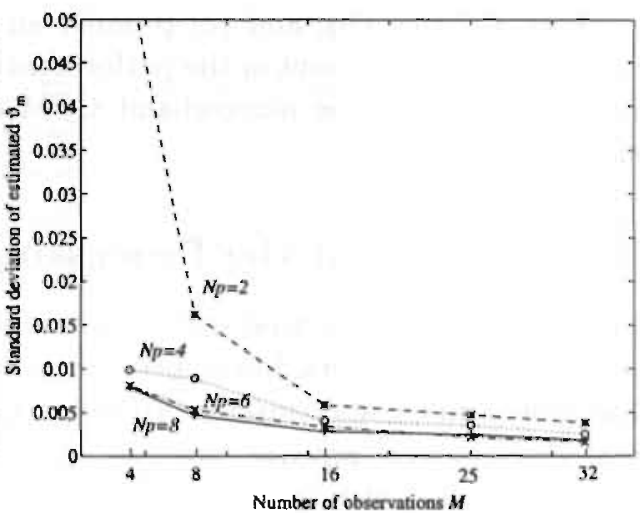

(d)

Figure 6.3. Influence of the depth and time window sizes. Four different lines represent four various sizes of depth window: the dashed line with stars pertains to $N_{\mathrm{p}}=$ 2 , the dotted line with circles to $N_{\mathrm{p}}=4$, the dashed-dotted line with crosses to $N_{\mathrm{p}}=6$, and the solid line with plus signs to $N_{\mathrm{p}}=8$. The estimates $\hat{\vartheta}_{\mathrm{m}}$ and standard deviations are plotted for two imposed scaled mean velocities: (a), (c) $\vartheta_{\mathrm{m}}=0.1 \lambda / T_{\text {prf }},(\mathrm{b}),(\mathrm{d}) \vartheta_{\mathrm{m}}=0.25 \lambda / T_{\text {prf }}$ as function of the time window size of $M=4,8,16,25$, and 32 observations. The other model parameters are kept constant: $\vartheta_{\mathrm{s}}=0, Q_{\mathrm{rf}}=2, \mathrm{SNR}=10 \mathrm{~dB}$. 


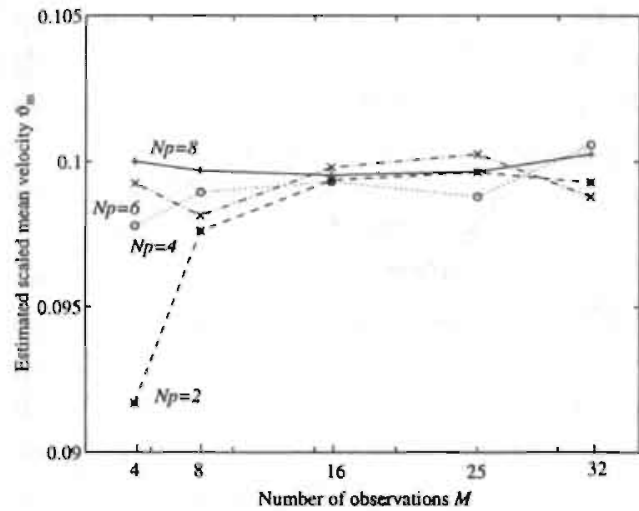

(a)

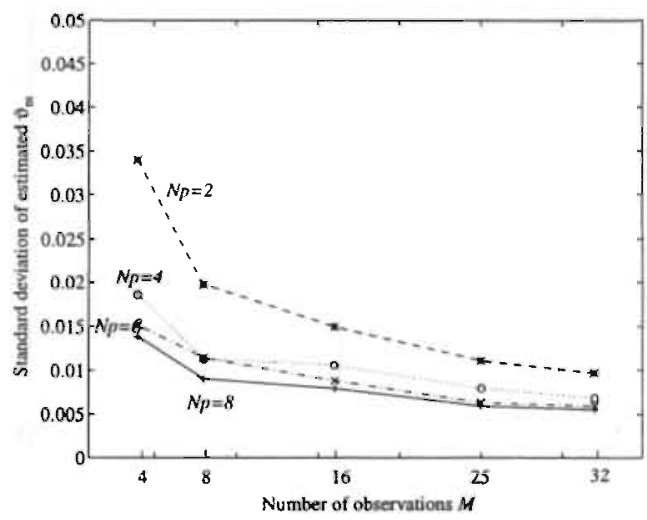

(c)

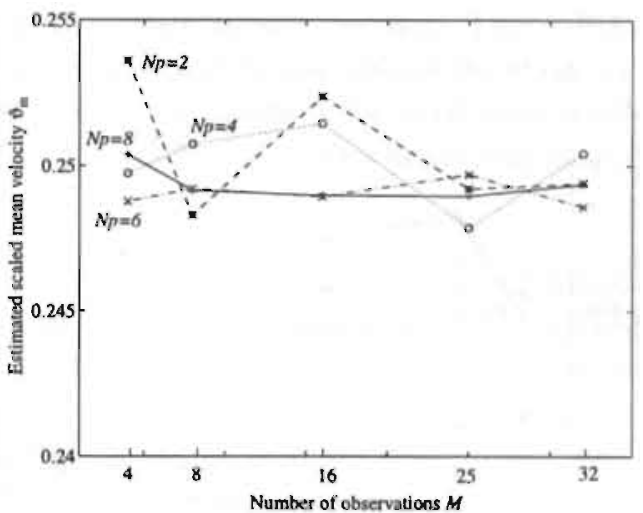

(b)

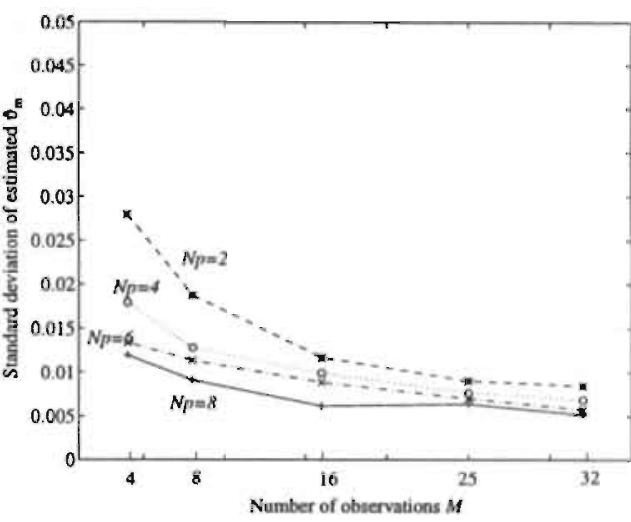

(d)

FiguRE 6.4. Effect of the velocity spread $\vartheta_{\mathrm{s}}=0.1 \lambda / T_{\text {prf }}$. Four different lines represent four various sizes of depth window: the dashed line with stars pertains to $N_{\mathrm{p}}=2$, the dotted with circles to $N_{\mathrm{p}}=4$, the dashed-dotted line with crosses to $N_{\mathrm{p}}=6$, and the solid line with plus signs to $N_{\mathrm{p}}=8$. The estimated scaled mean velocity $\hat{\vartheta}_{\mathrm{m}}$ and standard deviation are plotted for two imposed scaled mean velocities: (a), (c) $\vartheta_{\mathrm{m}}=0.1 \lambda / T_{\mathrm{prf}},(\mathrm{b})$, (d) $\vartheta_{\mathrm{m}}=0.25 \lambda / T_{\mathrm{prf}}$ as function of the time window size of $M=4,8,16,25$, and 32 observations. The other model parameters are not modified: $Q_{\mathrm{rf}}=2, \mathrm{SNR}=10 \mathrm{~dB}$. 


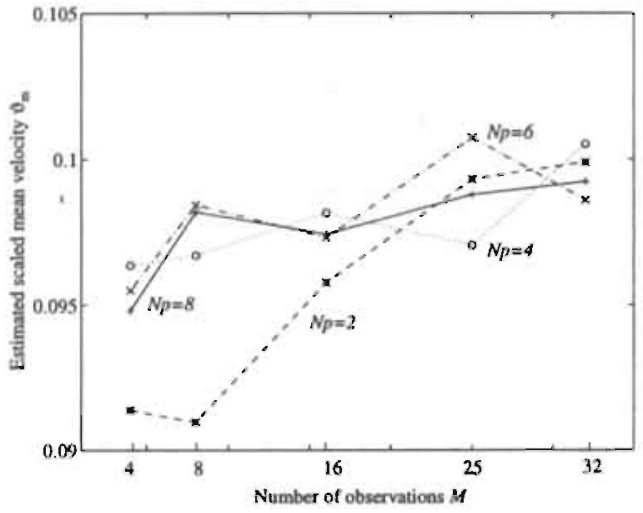

(a)

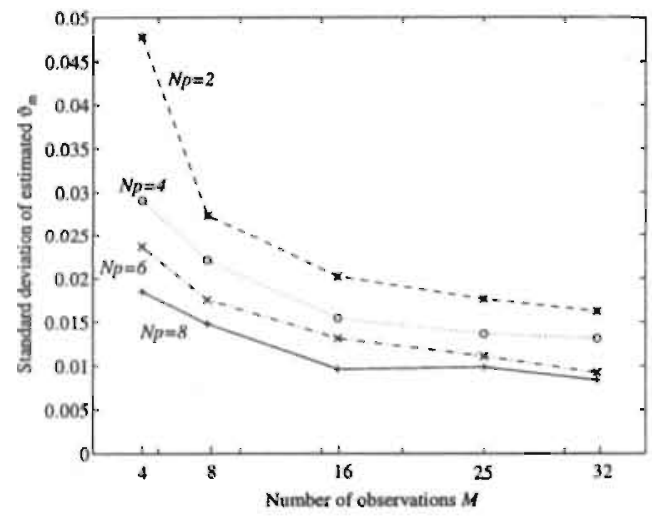

(c)

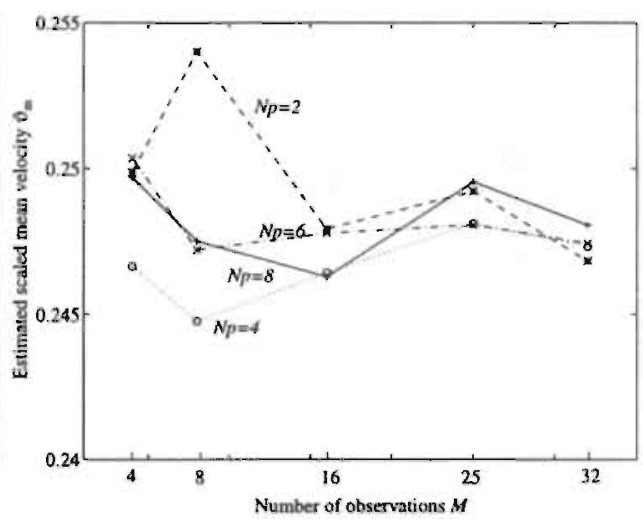

(b)

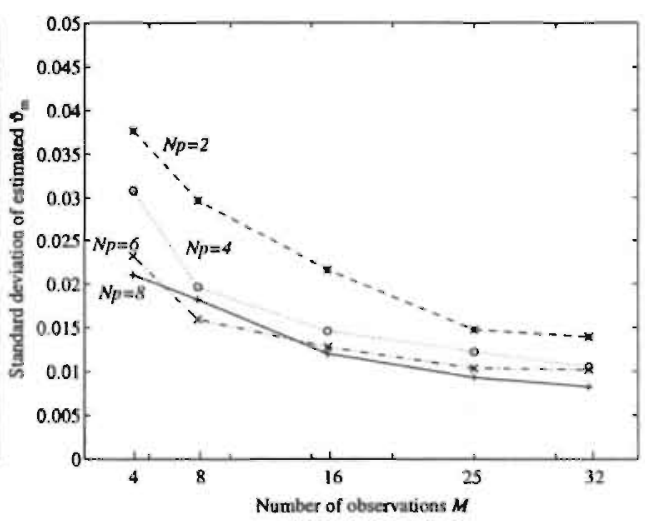

(d)

FIGURE 6.5. Effect of the velocity spread $\vartheta_{\mathrm{S}}=0.2 \lambda / T_{\mathrm{pr}}$. represent four various sizes of depth window: the dashed line with stars pertains to $N_{\mathrm{p}}=2$, the dotted line with circles to $N_{\mathrm{p}}=4$, the dashed-dotted line with crosses to $N_{\mathrm{p}}=6$, and the 'solid line with plus signs to $N_{\mathrm{p}}=8$. The estimated scaled mean velocity $\hat{\vartheta}_{\mathrm{m}}$ and standard deviation are plotted for two imposed scaled mean velocities: (a), (c) $\vartheta_{\mathrm{m}}=0.1 \lambda / T_{\mathrm{prf}},(\mathrm{b}),(\mathrm{d}) \vartheta_{\mathrm{m}}=0.25 \lambda / T_{\mathrm{prf}}$ as function of the time window size of $M=4,8,16,25$, and 32 observations. The other model parameters are not modified: $Q_{\mathrm{rf}}=2, \mathrm{SNR}=10 \mathrm{~dB}$. 
Let us have a look at Fig. 6.3 where zero velocity bandwidth is considered. Estimated mean values approach the true values, $0.1 \lambda / T_{\mathrm{prf}}$ in (a), and $0.25 \lambda / T_{\mathrm{prf}}$ in (b) as $N_{\mathrm{p}}$ and $M$ increase. The standard deviations in Figs. 6.3 (c) and (d) show monotone decreasing dependancy on $N_{\mathrm{p}}$ and $M$ as expected. When we inspect the two plotted lines for $N_{\mathrm{p}}=6$ and 8 , we see that they almost overlap. This means that a further increase of the length of depth window will do not reduce the standard deviation to such an extent that we would wish to exchange it for axial resolution. The stable behavior of the wideband CCM estimator shown in this figure will ensure good velocity precision and depth resolution.

Next, we will focus on the influence of imposed velocity bandwidth for conditions equivalent to those of the previous experiment (Fig. 6.3). Two different values were simulated: $\vartheta_{\mathrm{s}}=0.1 \lambda / T_{\mathrm{prf}}$, and $0.2 \lambda / T_{\mathrm{prf}}$. The resulting mean and standard deviation are demonstrated in Figs. 6.4, and 6.5. Because of identical conditions and selected zoom, the respective parts of these figures can be compared with each other as well as with Fig. 6.3, where zero velocity bandwidth was considered.

The estimated mean values undergo somewhat larger deviations for $M=4,8$ but with increased $M$ and $N_{\mathrm{p}}$ estimated means approach the true values (Figs. 6.4 (a), (b), 6.5 (a), (b)). An increase in standard deviation by a factor from 1.5 to 2 can be observed when the degree of velocity dispersion goes up in Figs. 6.4 (c), (d), 6.5 (c), (d). The results of these figures show again a steady. improvement in the standard deviation as the depth window becomes larger. The estimator also demonstrates a clear downward trend as more pulses become available for processing.

The results of this section offer the promise of excellent identification of blood velocity with an acceptable resolution also in the presence of velocity spread.

\subsubsection{Effect of the System Properties}

In this section the influence of system parameters such as signal to noise ratio (SNR) and quality fartor $\left(Q_{\mathrm{rf}}\right)$ is examined for the wideband $\mathrm{CCM}$ estimator.

Fig. 6.6 documents the effect of SNR for the low and high imposed scaled mean velocities: (a), (c) $\vartheta_{\mathrm{m}}=0.1 \lambda / T_{\mathrm{prf}}$, and (b), (d) $\vartheta_{\mathrm{m}}=0.3 \lambda / T_{\text {prf }}$. SNR varies from $0,5.10,15,40$, to $100 \mathrm{~dB}$, velocity bandwidth, quality factor and time window are fixed at the following values: $\vartheta_{\mathrm{s}}=0, Q_{\mathrm{rf}}=4, M=25$. The development of resulting mean and standard deviation is illustrated for two lengths of depth window, $N_{\mathrm{p}}=$ 4 in the dashed line with stars, and 8 periods in the solid line with plus signs.

In Fig. 6.6 we see that a change in SNR from $40 \mathrm{~dB}$ to $15 \mathrm{~dB}$ has little effect upon. bias and standard deviations. In contrast, a drop in SNR from $15 \mathrm{~dB}$ to $0 \mathrm{~dB}$ increases the statistical quality measures of performance (bias and standard deviation). Thus, above a threshold of $15 \mathrm{~dB}, \mathrm{SNR}$ has little effect upon the performance. At higher SNRs the quality of estimator does not change significantly with extension of the depth window. This is not surprising since the time window is large enough to ensure good performance. Check Fig. 6.3 at $M=25$. Longer $N_{\mathrm{p}}$ produces only slightly 


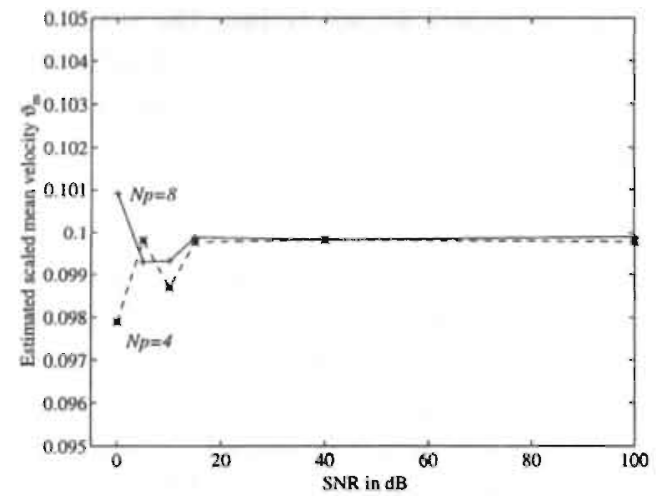

(a)

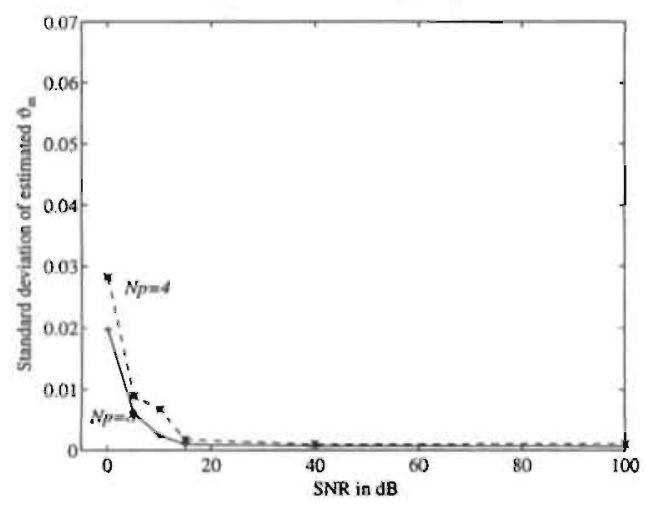

(c)

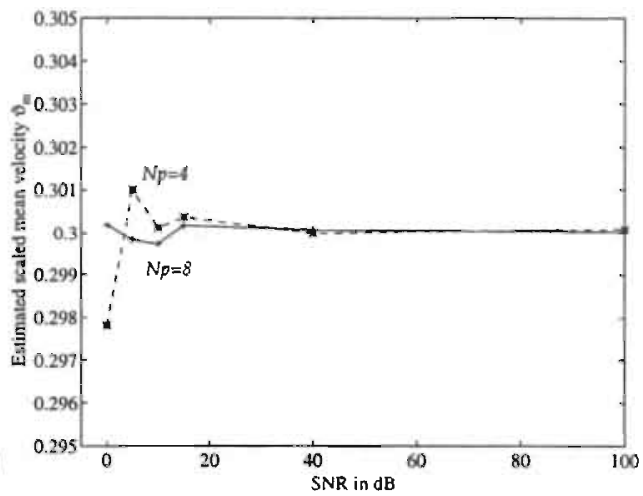

(b)

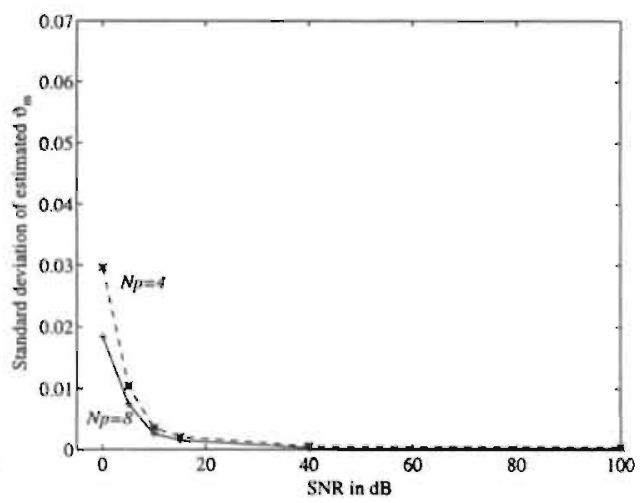

(d)

FIGURE 6.6. Effect of signal to noise ratio. Two different lines represent two various sizes of depth window: the dashed line with stars pertains to $N_{\mathrm{p}}=4$, and the solid line with plus signs to $N_{\mathrm{p}}=8$. The estimated scaled mean velocity $\hat{\vartheta}_{\mathrm{m}}$ and standard deviation are plotted for two imposed scaled mean velocities: (a), (c) $\vartheta_{\mathrm{m}}=0.1 \lambda / T_{\mathrm{prf}},(\mathrm{b})$, (d) $\vartheta_{\mathrm{m}}=0.3 \lambda / T_{\mathrm{prf}}$ as function of the signal to noise ratio SNR $=0,5,10,15,40,100 \mathrm{~dB}$. The other model parameters are kept constant: $\vartheta_{\mathrm{S}}$ $=0, Q_{\mathrm{rf}}=4, M=25$ observations. 
better results at $\mathrm{SNR}=10 \mathrm{~dB}$ at this point. It may be expected that this small difference will become insignificant at higher SNRs.

The effect of the quality factor on performance of the wideband CCM estimator was investigated under a typical SNR of $10 \mathrm{~dB}$. In Fig. 6.7 the estimated scaled mean velocities $\hat{\vartheta}_{\mathrm{m}}$ and their standard deviations are displayed for two imposed scaled mean velocities: (a), (c) $\vartheta_{\mathrm{m}}=0.1 \lambda / T_{\text {prf }}$, and (b), (d) $\vartheta_{\mathrm{m}}=0.25 \lambda / T_{\text {prf }}$ as a function of the quality factor $Q_{\mathrm{rf}}=2,4,6,8$. In this figure, the symbol $Q_{\mathrm{s}}$ means the same as $Q_{\mathrm{rf}}$ used in the text. We have included in (a) and (c) also the narrowband CCM estimator in order to compare it with respect to the $Q_{\mathrm{rf}}$. The wideband CCM estimator is shown in the dashed-dotted line with crosses for $N_{\mathrm{p}}=Q_{\mathrm{rf}}$, and the solid line with plus signs for $N_{\mathrm{p}}=2 Q_{\mathrm{rf}}$. The narrowband CCM estimator is drawn in the dotted line with circles for $N_{\mathrm{p}}=Q_{\mathrm{rf}}$, and in the dashed line with stars for $N_{\mathrm{p}}=2 Q_{\mathrm{rf}}$. The other settings are identical to those in Fig. 6.6.

Since in Section 2.3.1 it was shown that the quality factor determines the minimum number of periods, our choice for the size of depth window, $N_{\mathrm{p}}$, is expressed as integer multiple of $Q_{\mathrm{rf}}$. When we keep the ratio $N_{\mathrm{p}} / Q_{\mathrm{rf}}$ constant, and increase $Q_{\mathrm{rf}}$ (corresponding to elongation of the depth window as well as reduction of spatial resolution), the performance gets only slightly better (see Fig. 6.7).

In the sequel, we compare the narrowband and wideband CCM estimators in Fig. 6.7 (a) and (c). For $Q_{\mathrm{rf}}>6$ they all lie within the same range, which is however not true for a lower $Q_{\mathrm{rf}}$. We see the main difference in estimated mean at a quality factor of 2 (Fig. 6.7 (a)). The standard deviations are of the same order and can be decreased when the ratio $N_{\mathrm{p}} / Q_{\mathrm{rf}}$ increases. From this figure it is clear that a major advantage of the wideband CCM estimator is an excellent performance at a low $Q_{\mathrm{rf}}$ with a reasonable length of depth window. Employing a wideband signal together with short depth window allows for high axial (depth) resolution which is desirable in velocity imaging.

\subsubsection{Sensitivity Analysis for the Shape of the Spectrum}

To recapitulate, in Section 6.2, the wideband CCM estimator has been derived under the assumption of a symmetric RF spectrum. It is of interest to obtain an indication about the sensitivity of the estimator to this specific assumption. Computer simulations give us an opportunity to deliberately violate this assumption and generate the RF signal with a nonsymmetric spectrum. The proposed estimator is then applied to this signal to estimate the imposed mean velocity. Subsequent comparison of the performance gives us an indication of the robustness of the method in this specific aspect.

It means that the simulation model of the backscattered signal as described in Chapter 4 should be modified. It takes place at the second step of Section 4.4.1 where each data array is multiplied by the window given by (4.4.1). The original form of this window, plotted as a dashed line in Fig. 6.8 (a) should now be replaced by the sawtooth window, illustrated as a solid line in the same figure (this shape 


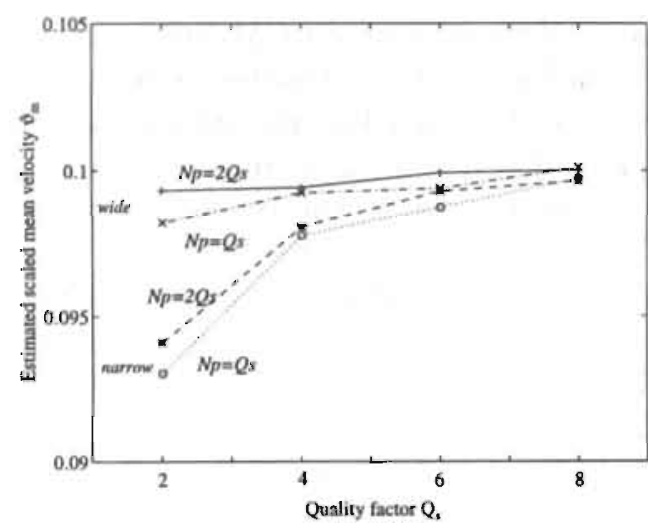

(a)

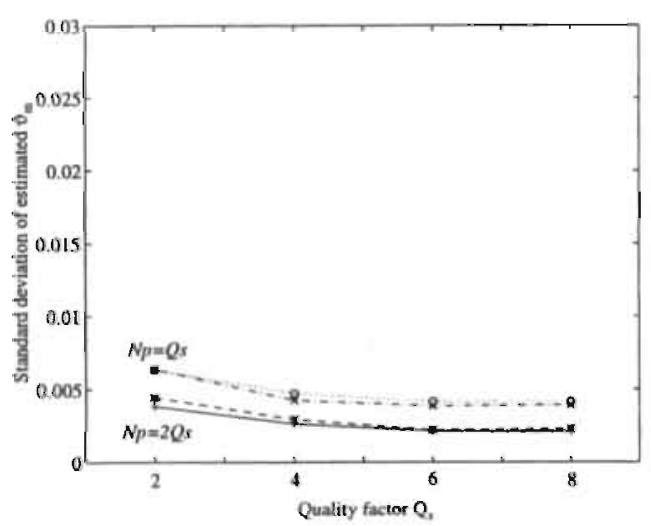

(c)

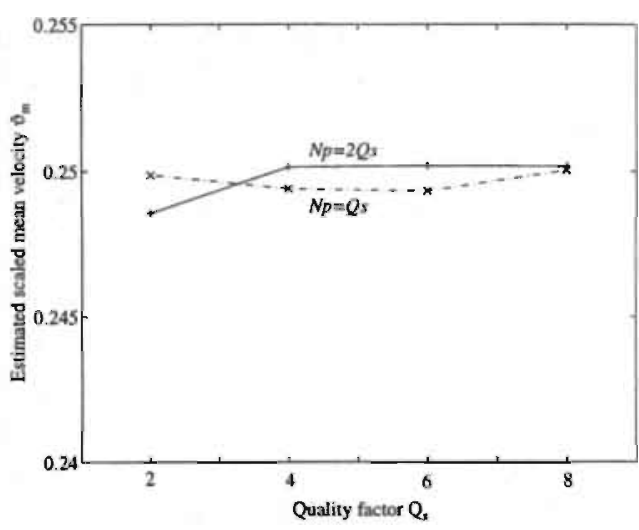

(b)

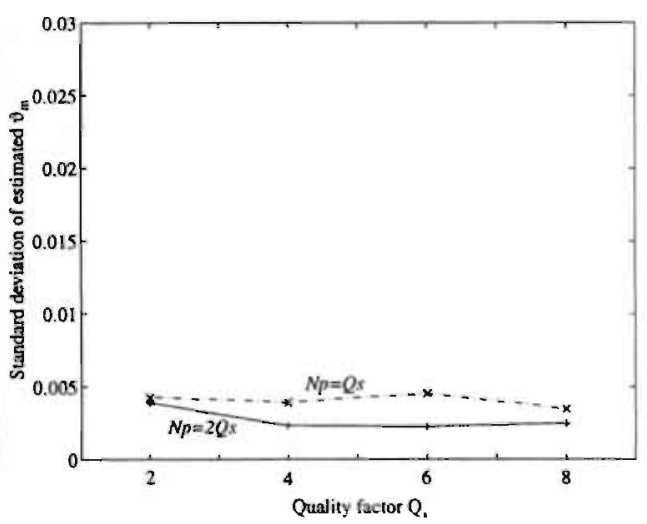

(d)

FigURE 6.7. Effect of quality factor. In this figure, the symbol $Q_{\mathrm{s}}$, means the same as $Q_{\mathrm{rf}}$ used in the text. The different lines represent two various sizes of depth window for the wideband or narrowband CCM estimator: the dashed line with stars pertains to the narrowband CCM estimator with $N_{\mathrm{p}}=2 Q_{\mathrm{rf}}$, the dotted line with circles to the narrowband CCM estimator with $N_{\mathrm{p}}=Q_{\mathrm{rf}}$, the dashed-dotted line with crosses to the wideband CCM estimator with $N_{\mathrm{p}}=Q_{\mathrm{rf}}$, and the solid with plus signs to the wideband CCM estimator with $N_{\mathrm{p}}=2 Q_{\mathrm{rf}}$. The estimated scaled mean velocities $\hat{\vartheta}_{\mathrm{m}}$ and standard deviations are plotted for two imposed scaled mean velocities: (a), (c) $\vartheta_{\mathrm{m}}=0.1 \lambda / T_{\mathrm{prf}}$, (b), (d) $\vartheta_{\mathrm{m}}=0.25 \lambda / T_{\mathrm{prf}}$ as function of the quality factor $Q_{\mathrm{rf}}=2,4,6,8$. The other model parameters are not modified: $M=25$ observations, $\vartheta_{\mathrm{s}}=0, \mathrm{SNR}=10 \mathrm{~dB}$. 


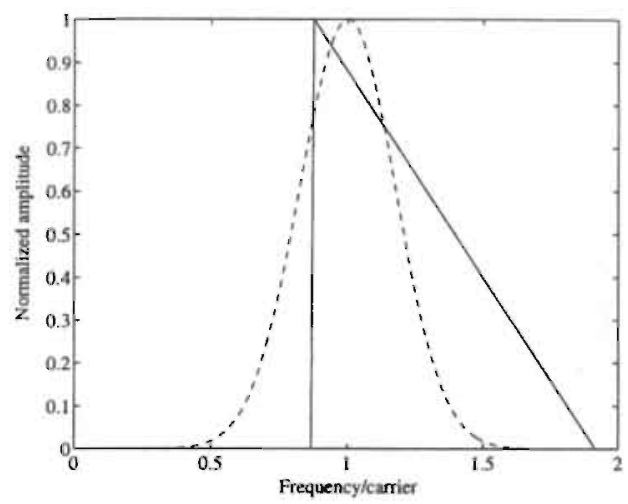

(a)

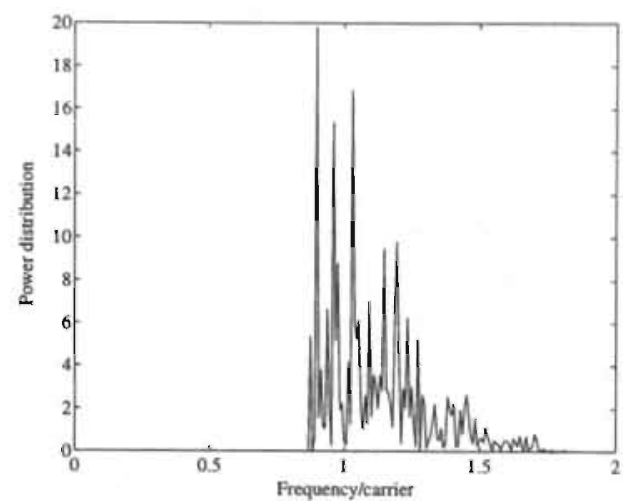

(b)

FIGURE 6.8. Generation of the RF signal with asymmetric spectrum. (a) shows the original Gaussian window in dashed line together with the asymmetric sawtooth window in solid line for $Q_{\mathrm{rf}}=4$. (b) depicts the power distribution of a generated waveform.

represents the worst case). The corresponding power distribution of a generated waveform can be seen in Fig. 6.8 (b) for $Q_{\mathrm{rf}}=4$. Having the generated sequence of the RF signals whose spectrum is nonsymmetric, they should be processed to obtain the wideband CCM estimates of the imposed scaled mean velocity as given by $(6.3 .22)$.

The results are depicted in Fig. 6.9 for the following defaults: $N_{\mathrm{p}}=4$ periods, $M$ $=25$ observations, $Q_{\mathrm{rf}}=4$, SNR $=10 \mathrm{~dB}$ as a function of the imposed scaled mean velocity, $\vartheta_{\mathrm{m}}$. As a reference, the statistics obtained from signals with the symmetric spectrum are given by crosses connected with a dashed-dotted line. Plus signs linked with a solid line correspond to statistics obtained from simulated signals with an asymmetric shape of the spectrum. Plots on the left-hand side, (a), (c) were simulated for a zero velocity spread, and plots on the right-hand side, (b), (d) for a $\vartheta_{\mathrm{s}}$ of $0.1 \lambda / T_{\text {prf }}$.

Looking at Fig. 6.9, our attention is drawn to particular figures at the scaled mean velocities $\vartheta_{\mathrm{m}}= \pm 0.4 \lambda / T_{\mathrm{prf}}$. An inspection of the sequence of estimates producing the rogue results has brought an insight into the cause of such large values in our quality measures. Within the sample set of 100 estimates, 1 to $3 \%$ of data was found with an opposite sign possibly due to the aliasing effects. These isolated sign errors have a disastrous effect on bias and standard deviation, fortunately, they can be easily detected and removed by for instance a median filter.

The subsequent median filtering (3-rd order filter) yielded bias and standard deviations dropping to values comparable with the reference. For instance, for the situation in Fig. 6.9 (a) the bias at $\vartheta_{\mathrm{m}}=-0.4 \lambda / T_{\text {prf }}$ dropped from 0.0256 to 0.0036 and the standard deviation from 0.1318 to 0.007 . Similar results were obtained for 


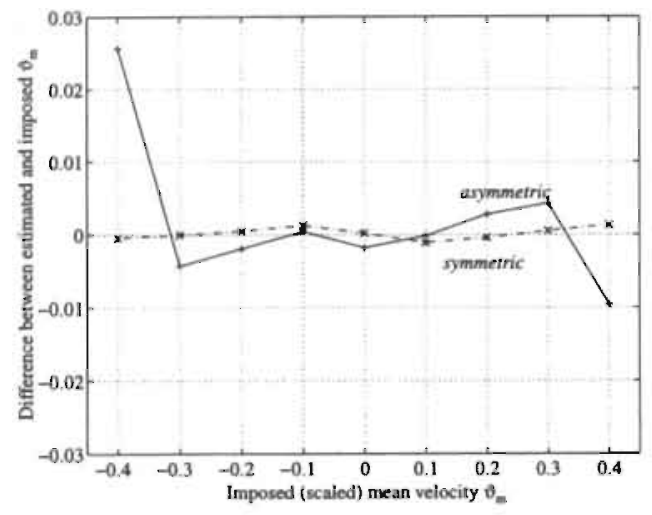

(a)

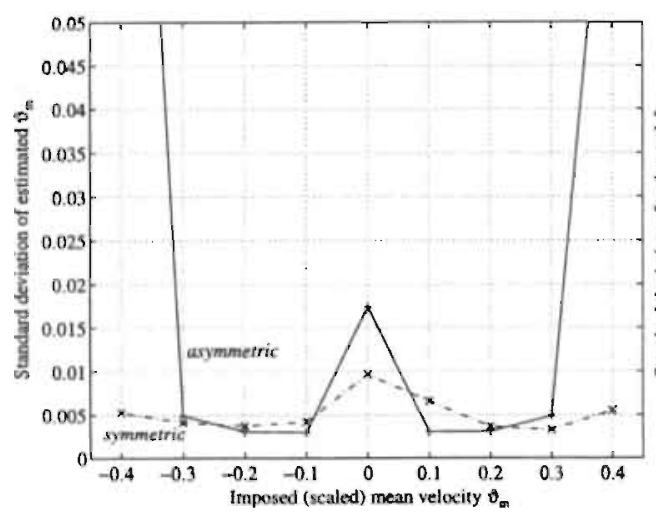

(c)

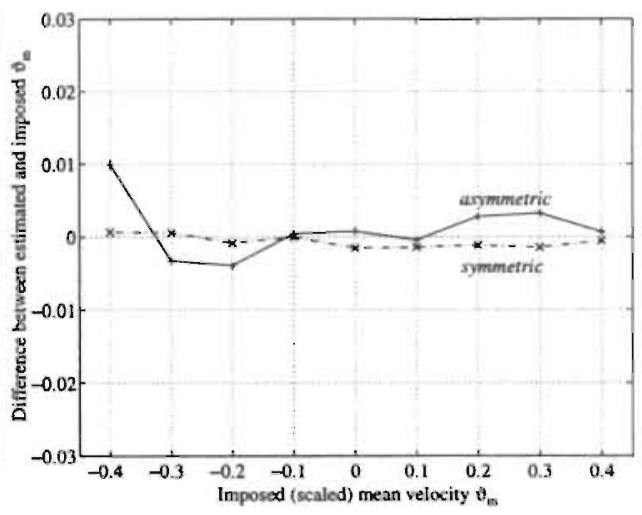

(b)

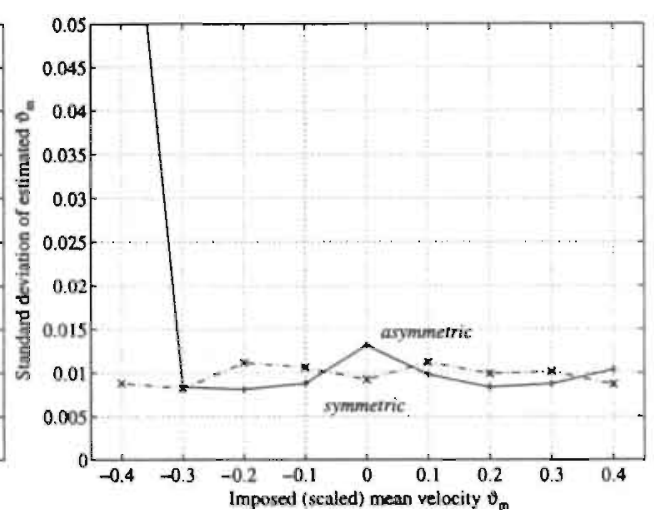

(d)

FIGURE 6.9. Sensitivity analysis for the shape of the spectrum. The bias in (a), (b) and standard deviations in (c), (d) are depicted as function of the imposed scaled mean velocity, $\vartheta_{\mathrm{m}}$. The solid line with plus signs corresponds to asymmetric shape of the spectrum whereas the dashed-dotted line with crosses to symmetric shape, taken as a reference. Plots on the left-hand side, (a), (c) were simulated for $\vartheta_{\mathrm{S}}=0$, and plots on the right-hand side, (b), (d) for $\vartheta_{\mathrm{s}}=0.1 \lambda / T_{\mathrm{prf}}$. The other model parameters are kept constant: $N_{\mathrm{p}}=4$ periods, $M=25$ observations, $Q_{\mathrm{rf}}=4, \mathrm{SNR}=10 \mathrm{~dB}$. 
other situations: in (a) for $\vartheta_{\mathrm{m}}=0.4 \lambda / T_{\mathrm{prf}}$ the bias changed from -0.0096 to -0.0025 and the standard deviation from 0.0787 to 0.0049 ; in (b) for $\vartheta_{\mathrm{m}}=-0.4 \lambda / T_{\mathrm{prf}}$ the bias improved from 0.01 to 0.0018 and the standard deviation from 0.081 to 0.0071 .

Hence, the assumption about the symmetric shape of the spectrum does not result in significant differences. The estimates obtained from signals that satisfy the assumption are comparable to those resulting from signals that do not comply with it. Performance is deteriorated by a sign error only at very high mean velocities $\vartheta_{\mathrm{m}}= \pm 0.4 \lambda / T_{\mathrm{prf}}$ which can be improved by a median filtering. Note, that the order of a filter does not need to be high, since only 1 to $3 \%$ of estimates were affected by an isolated sign error.

\subsubsection{Summary}

In this chapter we proposed and evaluated a new algorithm for velocity estimation that operates on high frequency RF data. On the basis of analysis with simulated signals presented in the previous sections $6.5 .1,6.5 .2,6.5 .3$, and 6.5 .4 we can make the following summary concerning the new wideband CCM estimator:

- The results indicate that the behavior of the wideband CCM estimator is stable for entire range of velocities and over a fairly wide range of signal and processing parameters. The observed deviations around zero velocity are not critical in velocity estimation. Zero velocities are usually suppressed by the wall filter and some velocity range within the sample volume will always be found. However, the observed deviation hampers application of this technique to tissue deformation studies. For tissue deformation the spatial derivative of the observed displacement will be considered and for a biased estimate this will produce large relative errors, especially if the observed velocity is close to zero. Of course, one may reduce the anticipated bias by taking long depth window but that will reduce the spatial resolution.

- The CCM approach does not demand the calculation of the complete correlation function. The wideband CCM estimator needs correlations at six lags, while correlations at five lags are required in the narrowband situation. The main advantage of the new estimator with respect to the narrowband CCM estimator is an improved performance at the low quality factors (less than 6). For signals with high quality factors, both estimators behave in a similar way.

- The method is robust to some extent in relation to an assumption of symmetric spectrum shape. At very high velocities an occasional sign error may occur due to the aliasing effects. Post-processing by median filter will remove this error.

Additional discussion of this method in relation to the maximum likelihood technique follows in Chapter 7. 



\section{Comparative Evaluation of Correlation Based Velocity Estimators}

\subsection{Introduction}

In the previous two chapters we have exclusively dealt with two estimation methods. In particular, we have given a comprehensive description and analysis of the maximum likelihood (ML) method and cross-correlation model (CCM) method.

The estimator described in Chapter 5 uses the maximum likelihood criterion. The estimation procedure has a certain affinity with a well known cross-correlation formula. The received signals are correlated with a known "reference" signal, and the position of the maximum of the absolute value of the correlation function yields the estimate. The second method, the cross-correlation model method introduced in Chapter 6, also employs the correlation formula. It is based on the modeling of the relationship between the successively received signals by the cross-correlation function. Fitting a curve through several lags of the generalized correlation function results in the estimate.

Clearly, a global framework that encompasses these two alternative schemes employs a generalized correlation function as defined in Section 3.3.1. Apart from the common aspect (correlation) of the methods mentioned, they belong to different categories within our classification scheme. The ML method falls into the category of methods that process the low-frequency signals, whereas the CCM technique is a member of class of methods that process high-frequency signals.

In the previous chapters both techniques have been developed in considerable detail, supported by an analysis of their performance. Most of the points discussed so far have been related to the precision aspects of each method as a function of the imposed processing and signal parameters. Yet, there are still several open questions as to which technique provides the best estimate for given processing conditions and how good the obtained estimate is with respect to the theoretical limit on performance. These questions cannot be addressed that easily by purely analytical methods, due to the complexity involved, so we shall again resort to computer simulations and comparison of the algorithms. The simulation procedure, as applied in Sections 5.4 


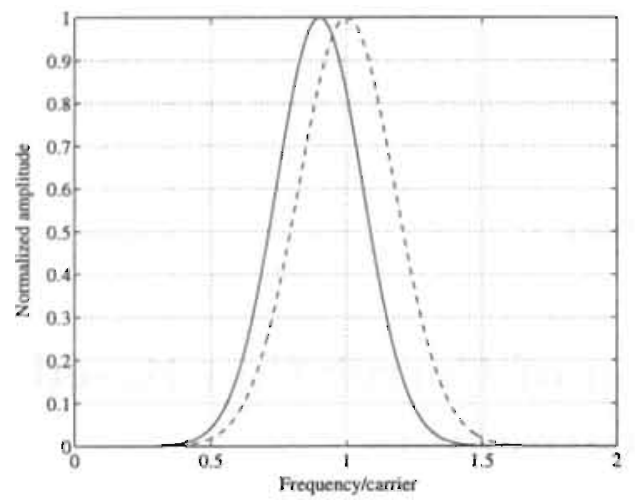

(a)

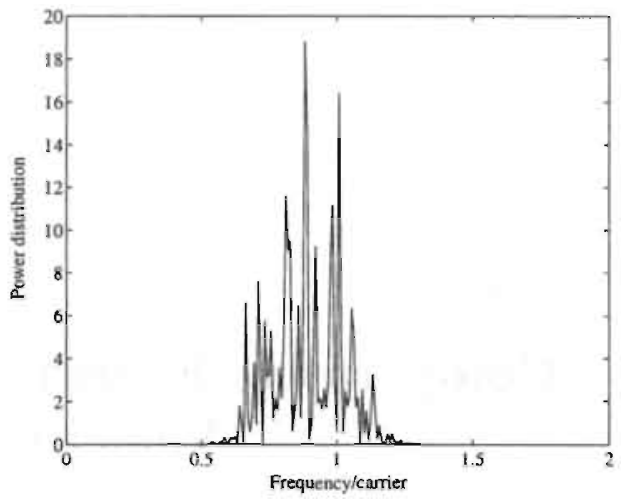

(b)

Figive 7.1. Generation of the RF signal under frequency-dependent attenuation. (a) shows the original Gaussian window as a dashed line together with shifted (at $90 \%$ of a carrier frequency) and narrowed window as a solid line for $Q_{\mathrm{rf}}=4$. (b) depicts a spectral power distribution of the generated waveform.

and 6.5 , was developed in such a way that without any significant modifications it can be adopted also for the purpose of this chapter.

The comparative evaluation of previously mentioned methods includes various aspects encountered in blood flow measurements. In Section 7.2 the quality of the estimators is investigated under frequency-dependent attenuation. It is indicated that this aspect is closely related to the character of input data. The efficiency of estimators is addressed in Section 7.3 more profoundly by inspecting the CramérRao lower bound which provides a theoretical limit to the performance of estimators. Section 7.4 presents an examination of the performance under typical operating conditions with an emphasis on precision, spatial and temporal resolutions, as well as on computational effort.

\subsection{Effect of Frequency Dependent Attenuation}

In this section we shall investigate the behavior of the ML and CCM estimators with respect to the frequency character of their input data, low frequency or high frequency. It involves tests on the effect of frequency-dependent attenuation produced by intervening tissue and by frequency-dependent scattering. The major effect of the frequency-dependent attenuation and scattering on the received signal is a shift in the spectral mean to lower frequencies as has been discussed in Section 2.4.3. Hence, to account for at tenuation effects, the mean frequency of the pulse should be decreased progressively with distance. What remains to be answered is how the quality of estimators is affected by frequency dependent attenuation, and how this aspect is related to the frequency character of input data. In the case of processing of low-frequency signals (ML method), the RF returns are transformed to low 
frequencies with respect to a reference frequency, thereby assuming that the spatial center frequency is equal to the emitted frequency (the associated complex envelope transformation was described in Section 3.4). This assumption implies that the frequency dependent attenuation is ignored in the processing procedure, which may degrade the performance of the estimator. In contrast, estimation methods that process high-frequency signals (CCM method) do not involve a signal transformation with respect to a reference frequency. Since no assumption needs to be taken on the spatial center frequency, its intrinsic estimation will prevent the deterioration of the estimator performance due to the frequency-dependent attenuation.

To test the above hypothesis, it was necessary to adapt the simulated backscattered signals that are obtained as an output of the simulation model of Chapter 4. A lower center frequency was adopted to emulate the phenomenon, keeping the quality factor $Q_{\mathrm{rf}}$ unchanged. This procedure is justified by our discussion in Section 2.4.3 and can easily be accounted for by skipping Assumption 4.5. Consequently, the Gaussian-shaped spectral window from the second step of Section 4.4 .1 will be modified as shown in Fig. 7.1 (a). The original window applied under Assumption 4.5 is plotted as a dashed line, whereas the window that simulates the frequency dependent attenuation is given as a solid line. The shape is the same, only its center is shifted to $90 \%$ of the original carrier frequency and the bandwidth is narrowed to maintain $Q_{\mathrm{rf}}=4$. An example of a spectral power distribution of an individual generated waveform is depicted in Fig. 7.1 (b).

In order to simulate the effect of frequency-dependent attenuation, the modifications mentioned above were applied in the simulation model of the RF signal from Chapter 4. The difference in performance is displayed in Fig. 7.2 where the maximum likelihood method (ML), plotted as a dashed line, represents the processing of lowfrequency signals, and the cross-correlation model method (CCM), plotted as a solid line, the processing of high-frequency signals. We see that the effect of frequencydependent attenuation (crosses for ML and circles for CCM) leads to a significant. bias in the ML velocity estimate. It approaches the expected error of $10 \%$ as quality factor $Q_{\text {rf }}$ goes up from 2, 4, 6, to 8 . The CCM velocity estimates almost overlap for both situations: for imposed scaled mean velocity $\vartheta_{\mathrm{m}}=0.1 \lambda / T_{\mathrm{prf}}$ in Fig. 7.2 (a), and for $\vartheta_{\mathrm{m}}=0.25 \lambda / T_{\mathrm{prf}}$ in Fig. 7.2 (b). Furthermore, the standard deviations (c), (d) are affected only by statistical inference (due to experimental uncertainties), and not by the effect of frequency-dependent attenuation. The operating conditions in Fig. 7.2 include: $N_{\mathrm{p}}=Q_{\mathrm{rf}}, M=25$ observations, $\vartheta_{\mathrm{s}}=0, \mathrm{SNR}=10 \mathrm{~dB}$.

The results of this section show that the maximum likelihood estimator suffers from the effect of frequency-dependent attenuation due to the fact which causes a significant bias in the estimate. In contrast, the cross-correlation model method estimator is not influenced by frequency-dependent effects. The difference in behavior can be explained on the basis of the frequency character of input data. Once again, the ML technique processes low-frequency signals which involves a signal transformation with respect to a reference frequency assumed to be the emitted frequency. Ap- 


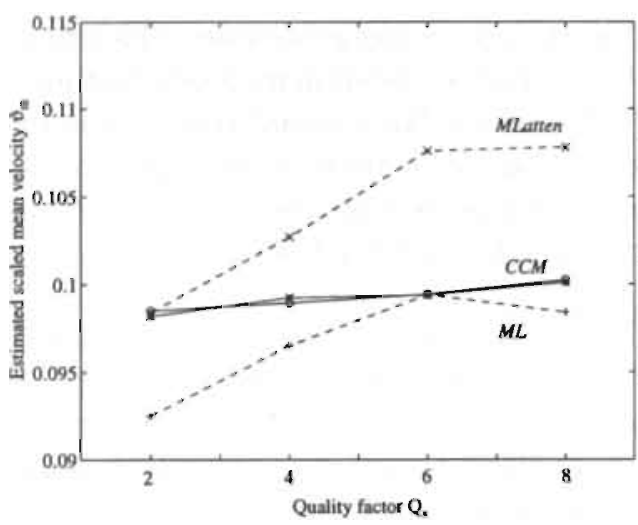

(a)

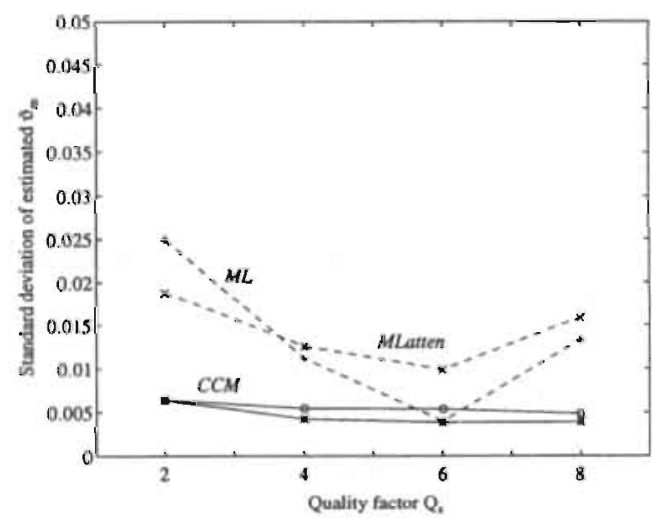

(c)

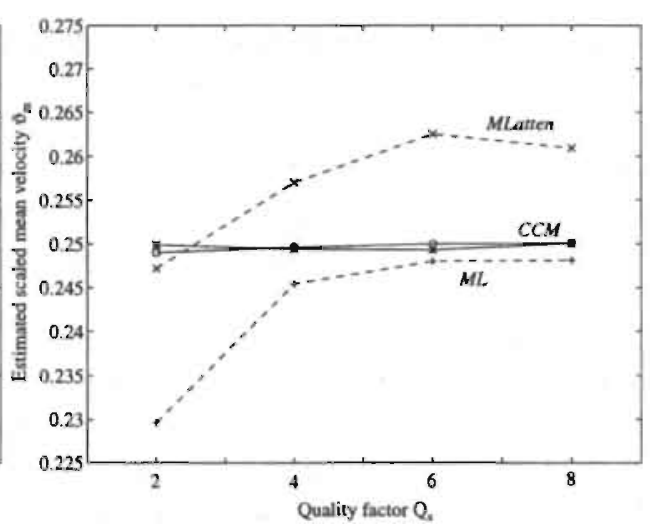

(b)

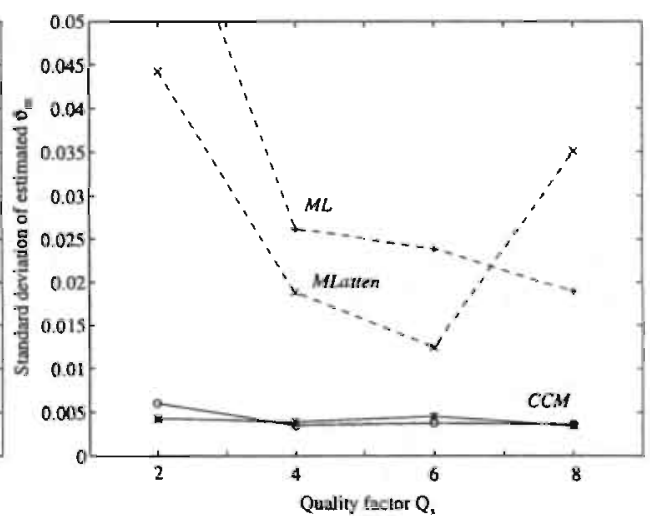

(d)

Figure 7.2. Effect of frequency dependent attenuation as function of the quality factor $Q_{\mathrm{rf}}$. In this figure, the symbol $Q_{\mathrm{s}}$ means the same as $Q_{\mathrm{rf}}$ used in the text. The different lines represent two estimation methods. The dashed line pertains to ML estimator where crosses is used for results with frequency dependent attenuation (MLatten) and plus signs for results without this effect (ML). The solid line represents wideband CCM method where circles denotes results with frequency dependent attenuation and stars denotes results without those effects. The estimated scaled mean velocities $\hat{\vartheta}_{\mathrm{m}}$ and standard deviations are plotted for two imposed scaled mean velocities: (a), (c) $\vartheta_{\mathrm{m}}=0.1 \lambda / T_{\text {prf }}$, (b), (d) $\vartheta_{\mathrm{m}}=0.25 \lambda / T_{\text {prf }}$ as function of the quality factor $Q_{\mathrm{rf}}=2,4,6,8$. The other model parameters are kept constant: $N_{\mathrm{p}}=Q_{\mathrm{rf}}, M=25$ observations, $\vartheta_{\mathrm{S}}=0, \mathrm{SNR}=10 \mathrm{~dB}$. 
proximate correction of the reference frequency for deep structures can be done by introducing the frequency-depth-dependent filter as described in [20], which complicates the implementation. Moreover, the correction is then based on assumptions rather than according to the actual signal characteristics. An elegant solution is to use the wideband CCM method that processes high frequency signals and is capable of estimating the carrier frequency. It circumvents the problem of assuming the reference frequency.

\subsection{Comparison of Performance with the Theoretical Limit}

A comparative evaluation of two correlation based estimators of velocity, the maximum likelihood method and the wideband cross-correlation model method is continued in this section by considering how the standard deviations of their velocity estimates relate to the theoretically expected limit on performance. The expression (7.3.5) provides a tool for such comparative quality assessment since its right-hand side is by definition the lower bound on standard deviation of any unbiased estimator, usually called the Cramér-Rao lower bound (CRLB).

We approach this problem in two steps. First, we derive the Cramér-Rao lower bound (CRLB) for any unbiased velocity estimator. We then show how the standard deviations of velocity estimates (obtained by two processing techniques) compare with this lower bound for different operating conditions. We discuss this comparison in detail for the maximum likelihood estimator and the wideband cross-correlation model estimator.

\section{Cramér-Rao Lower Bound for Velocity Estimator}

The derivation of CRLB is a straightforward application of the expression for the fundamental limit on the performance of a delay or displacement estimator developed by Walker in [68]. It is modified to a lower bound for velocity estimators and evaluated in our notation.

The starting point of the derivation of the lower bound for a velocity estimator is the minimum variance of any unbiased estimator of displacement [68] given by:

$$
\sigma^{2} \geq \frac{3 c}{2 \pi^{2} f_{\mathrm{c}}^{3} N_{\mathrm{p}} x_{\mathrm{s}}\left(Q_{\mathrm{rf}}^{-3}+12 Q_{\mathrm{rf}}^{-1}\right)}\left(\frac{1}{\rho_{\mathrm{d}}^{2}}\left(1+\frac{1}{\mathrm{SNR}_{\text {ratio }}}\right)^{2}-1\right),
$$

where $\Delta x$ is the true displacement, $\Delta \hat{x}$ is the estimated displacement and $x_{\mathrm{s}}$ is the sampling distance. We note that the variance (7.3.1) is not simply proportional to the inverse root of the bandwidth $\left(Q_{\mathrm{rf}} / f_{\mathrm{c}}\right)$, as is often stated [23], [63]. The inequality (7.3.1) demonstrates that it is a function of signal and processing characteristics: center frequency, $f_{\mathrm{c}}$, quality factor of the signal, $Q_{\mathrm{rf}}$ (which includes information on the bandwidth), signal-to-noise ratio, $\mathrm{SNR}_{\text {ratio }}$ (defined by equation 2.4.17), and window length $N_{\mathrm{p}}$. Under Assumptions 4.1 to 4.6, the center frequency, $f_{\mathrm{c}}$, is in our 
notation equal to

$$
f_{c}=\frac{1}{2} \Psi_{e} \xi_{\mathrm{s}} c
$$

where relation (2.4.4) was applied with spatial frequency $\xi_{\mathrm{e}}=\Psi_{\mathrm{e}} \xi_{\mathrm{s}}\left(\Psi_{\mathrm{e}}\right.$ is a normalized spatial center frequency, $\xi_{\mathrm{s}}$ is a sampling frequency in depth and $c$ is the acoustic speed).

In (7.3.1), $\rho_{\mathrm{d}}$ is the correlation coefficient that reflects decorrelation by physical processes due to the relative motion of scatterers within the observation window. In the limit of perfect signal correlation, $\rho_{\mathrm{d}}=1$, the expression (7.3.1) gives the Cramér-Rao lower bound which agrees with those derived in [39], [54]. The inequality (7.3.1) was found for identically shaped bandlimited spectra for both noise and signal. It is not particularly restricted to that form of spectrum. A rectangular or Gaussian shape yields the same result.

The next step is to propose a CRLB for any unbiased velocity estimator. To recapitulate, a pulsed Doppler instrument measures the velocity of scatterers as the displacement between the pulse repetition period, $T_{\text {prf }}$, and therefore,

$$
E\left[\left(\hat{\vartheta}_{\mathrm{m}}-\vartheta_{\mathrm{m}}\right)^{2}\right]=\frac{1}{T_{\mathrm{prf}}^{2}} E\left[(\Delta \hat{x}-\Delta x)^{2}\right]
$$

where $\hat{\vartheta}_{\mathrm{m}}$ is the estimated scaled mean velocity and $\vartheta_{\mathrm{m}}$ is the true scaled mean velocity. The standard deviation is more useful than the variance when it comes to presenting results, because it has the same unit as the original quantity. For the standard deviation of the velocity estimator, the Cramér-Rao inequality (3.3.10) holds for the square root of the lower bound, hence

$$
\sigma=\sqrt{E\left[\left(\hat{\vartheta}_{\mathrm{m}}-\vartheta_{\mathrm{m}}\right)^{2}\right]} \geq \mathcal{F}\left(\vartheta_{\mathrm{m}}\right)^{-\frac{1}{2}}
$$

Substitution of (7.3.2), (7.3.1) with $\rho_{\mathrm{d}}=1$, and (7.3.3) into (7.3.4) results in the Cramér-Rao inequality for the standard deviation of any unbiased velocity estimator

$$
\sigma \geq \frac{1}{T_{\text {prf }}}\left(\frac{12}{\pi^{2} c^{2} \xi_{\mathrm{s}}^{2} \Psi_{e}^{3} N_{\mathrm{p}}\left(Q_{\mathrm{rf}}^{-3}+12 Q_{\mathrm{rf}}^{-1}\right)}\left(\left(1+\frac{1}{\mathrm{SNR}_{\text {ratio }}}\right)^{2}-1\right)\right)^{-\frac{1}{2}} .
$$

The Cramer-Rao lower bound (7.3.5) is a best-case lower bound in the sense that it assumes that the spectra of signal and noise are identical and bandlimited, signals are perfectly correlated (no decorrelation occurs due to the physical processes). Independently of the veracity of these assumptions, the significance of the CRLB resides in the fact that it provides a theoretical limit to the performance of the processing techniques. We can compare them with the Cramér-Rao lower bound (7.3.5) and determine whether and how close they may approach the theoretical limit for different operating conditions. This question cannot be addressed so easily by purely analytical methods due to the complexity involved. As already mentioned in the 
previous sections, such an attempt would require a linearization procedure that may lead to oversimplification and the final expressions would no longer correctly describe the behavior of an estimator. Therefore, it is more attractive to inspect the relation to the theoretical limit by means of the simulation procedure, which ensures that effects of different processing conditions are likely to show up.

\section{Comparison of ML and CCM estimators with CRLB}

The simulation environment for the purpose of this section is equivalent for both techniques (ML and wideband CCM) and was already tested in Sections 5.4 and 6.5. The standard deviations of the $\mathrm{ML}$ and wideband $\mathrm{CCM}$ estimators obtained in the performance evaluation are now compared against the Cramér-Rao lower bound (7.3.5). A default choice of simulation model parameters in (7.3.5), includes sampling quantities, $T_{\mathrm{prf}}, \xi_{\mathrm{s}}$, that are equal to unity. Hence in accordance with (3.2.1) the normalized spatial center frequency $\Psi_{\mathrm{e}}$ equals $1 / 4$. Acoustic speed $c$ is taken to be $1540 \mathrm{~ms}^{-1}$. It interest us what is the ratio of standard deviations obtained by the ML or wideband CCM method and standard deviations given by the CramérRao lower bound (7.3.5). In this section the shortcut CCM represents the wideband cross-correlation model method. We shall consider various operating conditions.

The standard deviations compared to CRLB as a function of the signal-to-noise ratio SNR are depicted in Fig. 7.3. The standard deviations are plotted for two imposed scaled mean velocities: (a), (c) $\vartheta_{\mathrm{m}}=0.1 \lambda / T_{\mathrm{prf}}$, (b), (d) $\vartheta_{\mathrm{m}}=0.3 \lambda / T_{\mathrm{prf}}$ for signal-to-noise ratios of $\mathrm{SNR}=0,5,10,15$, and $40 \mathrm{~dB}$.

Overall, the CCM/CRLB ratios have smaller values than have the ML/CRLB ratios. The ratio CCM/CRLB varies from 6 to 2, where the largest value occurs at a $\mathrm{SNR}$ of $0 \mathrm{~dB}$. In contrast, the ratio $\mathrm{ML} / \mathrm{CRLB}$ at a SNR of $0 \mathrm{~dB}$ reaches values hard to deduce from the plots, especially when measuring high velocities as seen in Figs. 7.3 (c), (d). This is not surprising, because the ML method under the considered processing conditions is biased at high. velocities $\left(>0.2 \lambda / T_{\text {prf }}\right)$ as was shown in Section 5.4, nevertheless we included this situation for completeness. Remember, the CRLB is by definition a lower bound on standard deviation of an unbiased estimator. When we inspect the situation at low velocity, in Figs. 7.3 (a), (b), the ratio ML/CRLB is at a SNR of $0 \mathrm{~dB}$ approximately 20 , which is more than 3 times larger than the corresponding $C C M / C R L B$ ratio. The dependence of the ML standard deviation on the signal-to-noise ratio is obvious and was explained in Section 5.3. Only at higher SNRs does the ML/CRLB ratio stabilize at values like 8, and 5 when considering low velocity. An improvement can be achieved by applying a longer depth window which is observable between Fig. 7.3 (a) for $N_{\mathrm{p}}=Q_{\mathrm{rf}}$ and Fig. 7.3 (c) for $N_{\mathrm{p}}=2 Q_{\mathrm{rf}}$. Analysis of the situation of longer depth window for the case of the wideband CCM indicates that the CRLB is a tight bound, where the CCM/CRLB ratio is approximately 2 to 1,5 . This is, in contrast to the ML case, also valid at high velocity (Figs. 7.3 (c), (d)). 


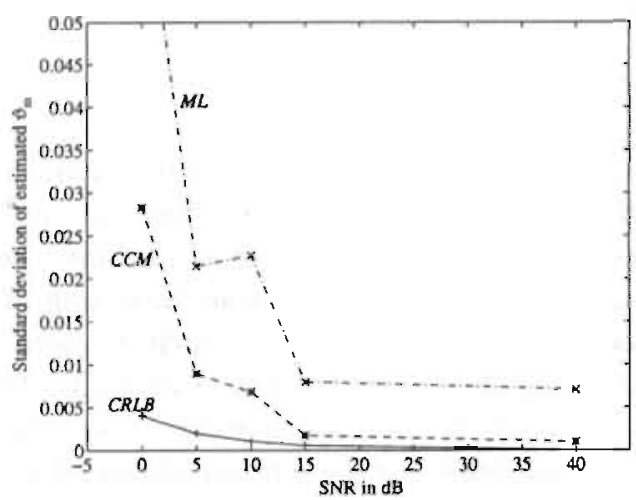

(a)

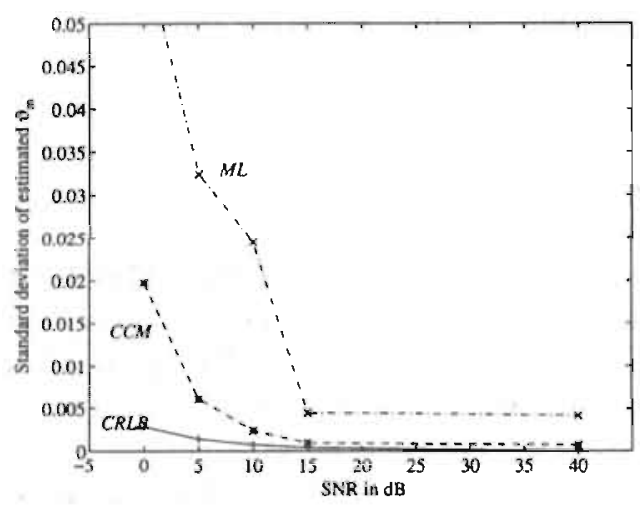

(c)

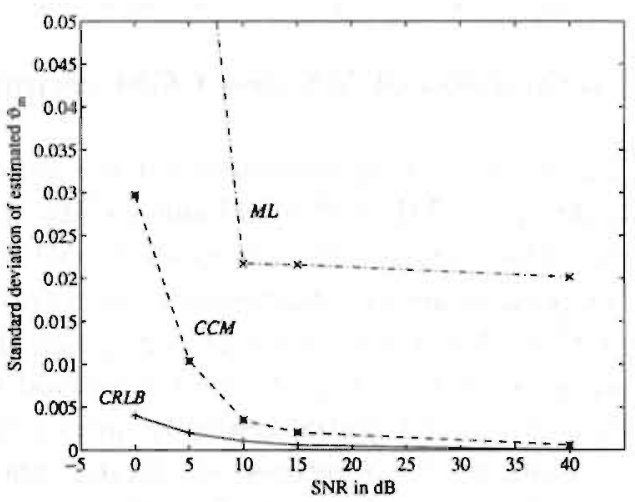

(b)

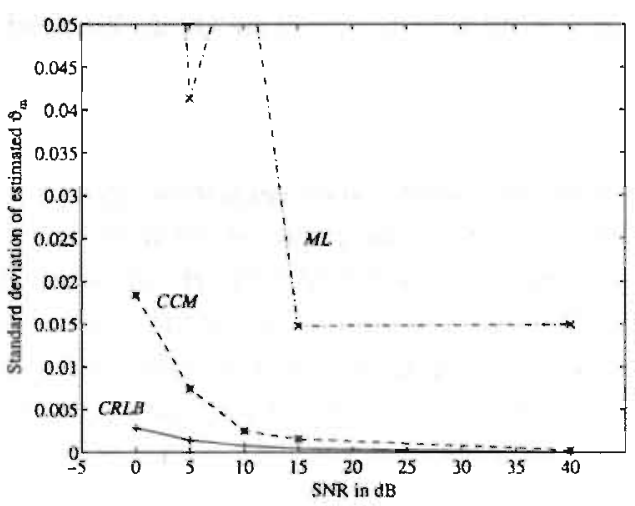

(d)

FIGURE 7.3. Comparison of the performance with the theoretical limit as function of the SNR. The standard deviations are compared for two imposed scaled mean velocities: (a), (c) $\vartheta_{\mathrm{m}}=0.1 \lambda / T_{\mathrm{prf}}$, (b), (d) $\vartheta_{\mathrm{m}}=0.3 \lambda / T_{\mathrm{prf}}$ for signal to noise ratios of SNR $=0,5,10,15$, and $40 \mathrm{~dB}$. The solid line with plus signs represents the CRLB, the dashed line with stars pertains to the wideband CCM estimator and the dasheddotted line with crosses to the ML estimator. Two different lengths of depth window are considered: in (a), (b) $N_{\mathrm{p}}=Q_{\mathrm{rf}}$, and in (c), (d) $N_{\mathrm{p}}=2 Q_{\mathrm{rf}}$. The other model parameters are not inodified: $\vartheta_{\mathrm{s}}=0, Q_{\mathrm{rf}}=4, M=25$ observations. 


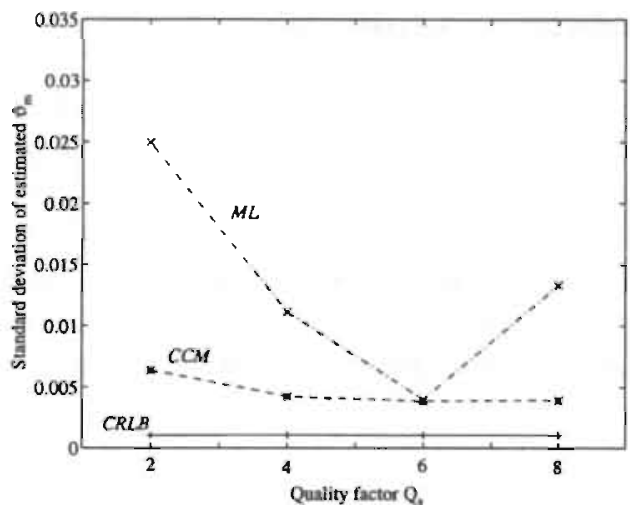

(a)

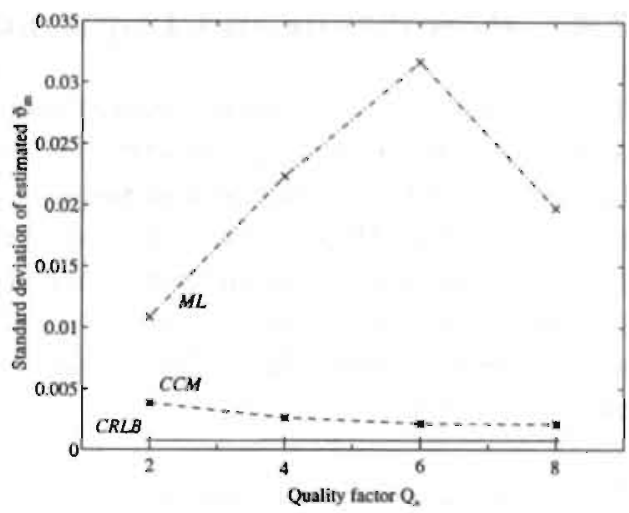

(b)

Flgure 7.4. Comparison of the performance with the theoretical limit as function of quality factor and indirectly also as function of the depth window size: in (a) $N_{\mathrm{p}}=Q_{\mathrm{rf}}$, and in (b) $N_{\mathrm{p}}=2 Q_{\mathrm{rf}}$. In this figure, the symbol $Q_{\mathrm{s}}$ means the same as $Q_{\mathrm{rf}}$ used in the text. The standard deviations are compared for quality factor $Q_{\mathrm{rf}}=2,4,6,8$. The solid line with 'plus signs' represents the CRLB, the dashed line with 'stars' pertains to the wideband CCM estimator and the dashed-dotted line with crosses to the ML estimator. The other model parameters are not modified: $\vartheta_{\mathrm{m}}=0.1 \lambda / T_{\mathrm{prf}}, \vartheta_{\mathrm{s}}=0, M=25$ observations, $\mathrm{SNR}=10 \mathrm{~dB}$.

Fig. 7.4 provides another perspective on comparison of the CCM and ML standard deviations with the theoretical limit given by CRI.B. Here, only low imposed mean velocity is considered $\left(\vartheta_{\mathrm{m}}=0.1 \lambda / T_{\mathrm{prf}}\right)$, and the ML and CCM standard deviations are compared to CRLB as a function of the quality factor $Q_{\mathrm{rf}}$ that varies from 2, 4, 6 , to 8 and indirectly as a function of the depth window size: in (a) $N_{\mathrm{p}}=Q_{\mathrm{rf}}$, and in (b) $N_{\mathrm{p}}=2 Q_{\mathrm{rf}}$. Consequently, the CRLB is constant for the conditions in this figure. Note that the symbol $Q_{\mathrm{s}}$ in the figure means the same as $Q_{\mathrm{rf}}$ used in the t.ext. It is of interest to speculate on estimation error at a SNR typical for blood flow conditions, which is selected at $10 \mathrm{~dB}$ in Fig. 7.4. This is actually a poor SNR, thus the the ML/CRLB ratio fluctuates between 31 and 5 as a function of the quality factor as well as a function of the depth window size. As far as the wideband CCM is concerned, we observe that a longer depth window size causes a decrease of the CCM/CRLB ratio by factor 2 .

The comparative evaluation of the standard deviations of the two reported methods with respect to the theoretical lower bound (CRLB) has proven that the wideband CCM is an efficient estimator (in the sense defined in Section 3.3.3) for all processing conditions considered. The coefficient of efficiency (CRLB/CCM $100 \%$ ) gets better as signal-to-noise ratio and the size of depth window increase. Achieved values of the efficiency coefficient of the wideband CCM estimator have varied from $17 \%$ to $70 \%$ under the conditions considered in this sections. 


\subsection{Resolution and Implementation Aspects}

The performance in terms of bias and standard deviation was evaluated as a function of signal and operating parameters for each technique separately, in Section 5.4 for the maximum likelihood method and in Section 6.5 for the wideband cross-correlation model method. Here, we are interested in comparison of these techniques with respect to each other. Our comparative analysis in this section is based on results from the previous two chapters, representing both methods in comparable diagrams. For all results considered here, the signal to noise ratio is kept at a constant realistic value of $10 \mathrm{~dB}$.

To put the algorithms in perspective, let us compare Figs. 5.5 (a), (b), (c) and Figs. 6.2 (a), (b), (c) where the difference between estimated and true velocity is plotted together with the $95 \%$ confidence interval. Notice, that (a), (b), and (c) conditions are identical in both figures mentioned. The selected picture sizes are identical too, which makes a visual comparison very clear. Looking at the figures, we see that the results for both methods differ to a large extent. The behavior of the wideband CCM estimator is very stable for the whole range of velocities. However, the opposite is true for the maximum likelihood estimator. Higher velocities are significantly biased and the dispersion of estimates is approximately 5 to 10 times larger than the spread of the wideband CCM estimates. The results with the ML method do not approach the results with the wideband CCM method, even when the ML estimates are filtered with 7-th order median filter (see Figs. 5.5 (d) and 6.2 (c)). These observations from Figs. 5.5, and 6.2 indicate that the performance of the wideband CCM technique is superior to that of the maximum likelihood method.

A further investigation in the following subsections, concerning resolution and implementation aspects, should confirm the above statement. The precision of both methods will be compared in view of the underlying operating resolution (axial in Section 7.4.1, temporal in Section 7.4.2) since these characteristics (i.e. precision and resolution) can usually be improved at the expense of one another. An implementation aspect will be discussed in Section 7.4.3, considering the computational effort involved in a real-time realization.

Resolution and implementation aspects are important for the technology known as color flow imaging. It attempts to estimate the blood velocity field and present the results as a color coded information superimposed on grey-level anatomical information provided by B-mode echo image. More about the effect of these aspects on color flow imaging can be found in [13].

\subsubsection{Precision and Axial Resolution}

The spatial resolution as defined in Section 2.3.2, is determined by the echo-signal properties and the processing parameters. It is linked to the ultrasonic axial resolution, which in turn is related to the pulse length. Thus axial resolution in ultrasound 
instrumentation can be approximated by the quality factor as was explained in Section 2.3.2. It is logical to expect that the axial resolution is smeared by observation windows in depth $\left(N_{\mathrm{p}}\right)$ longer than the pulse length yielding spatially averaged estimates of scatterer velocity. Hence, the quality factor, $Q_{\mathrm{rf}}$, and size of the depth window, $N_{\mathrm{p}}$, are in this context the most important factors. Therefore, in this section we will compare the maximum likelihood (ML) estimator to the wideband cross-correlation model (CCM) estimator with respect to those parameters.

First, Fig. 5.10 (ML unfiltered estimates) and Fig. 6.7 (wideband CCM estimates) are examined as a function of the quality factor. It is assumed that the observation window is not smaller than the ultrasonic pulse length, and is chosen in accordance with quality factor: $N_{\mathrm{p}}=Q_{\mathrm{rf}}$ or $2 Q_{\mathrm{rf}}$. Again, (a), (b), (c), and (d) conditions and zoom windows are identical for both figures. Therefore, it is easy to see that quality measures for the wideband CCM method are much better than for the ML method. A velocity estimated by the wideband CCM method closely follows the true velocity (low bias) with a small standard deviation. The ML estimates exhibit larger deviations from the true value and unpredictable standard deviations, especially at higher velocities.

In general, precision improves for higher quality factors. We also observe that if the size of the depth window is doubled, the precision of both methods becomes better. This effect is more pronounced in Figs. 5.6, 5.7, 5.8, and Figs. 6.3, 6.4, 6.5. They are plotted for a low quality factor, $Q_{\mathrm{rf}}=2$, for different sizes of observation window. Without doubt, for both methods considered the precision at fixed $M$ improves as the size of the depth window is enlarged. Since we are interested in a good axia] resolution, smaller window sizes are more attractive to investigate. Then looking at Figs. 5.6 and 6.3 we see that for $N_{\mathrm{p}}=2$, or 4 , e.g. $N_{\mathrm{p}}=Q_{\mathrm{rf}}$, or $2 Q_{\mathrm{rf}}$, the precision of the wideband CCM is superior to that of the ML method. The difference between estimated and true velocity is much smaller for the wideband CCM estimates. On the other hand, the ML estimates are biased when a. small depth window is applied and the standard deviation is larger, approximately by a factor of 10 . This factor rises further in the presence of different velocity components, because the ML estimator is very sensitive to velocity dispersion (compare Fig. 5.7 with 6.4, and Fig. 5.8 with 6.5 ).

Consequently, we can make the following observations concerning precision and resolution based on the above discussion:

- a low quality factor enlarges signal bandwidth, which would result in improvement of the axial resolution but not precision improvement;

- an increasing size of the observation window improves precision, and at the same time decreases spatial resolution.

This suggests, not surprisingly, that the optimal design of a velocity estimator would encompass systems with a low quality factor and short observation windows. Under 
these desirable conditions the wideband CCM estimator provides more precise estimates than does the ML estimator. The ML method is unrealible in conditions of higher mean velocities and in the presence of velocity dispersion.

\subsubsection{Precision and Temporal Resolution}

Temporal resolution in the context of imaging is usually defined as frame rate, i.e. the number of frames recorded per time unit. We adopt this definition since velocity information is usually visualized, for example by color coded imaging, mentioned in Section 7.4, where a smooth and continuous presentation of blood flow images is desirable. However, temporal stationarity of the RF signal as discussed in Section 2.4.1 may cause discontinuities in the flow information obtained. In order to receive an adequate picture of dynamic blood flow conditions, the number of estimates of velocity per time unit should be large.

Logically, the temporal resolution is determined by the number of RF signals used per estimate, i.e. the size of the time window. As we know, individual observations are obtained at an interval equal to the pulse repetition period, and if more observations are processed per estimate, the rate of estimates per time unit will decrease.

Now, it is clear that an important factor in the context of temporal resolution is the size $M$ of the time window with respect to which the proposed estimation methods are evaluated. In this section, comparison is based on results presented in figures in the previous two chapters for the maximum likelihood method (ML) and the wideband cross-correlation model (CCM) method, for identical conditions and picture sizes. Some results for the ML estimator lie therefore outside the selected picture size.

Looking at Figs. 5.6 and 6.3 we see that for a fixed depth window the precision (bias and standard deviation) improves with increasing $M$. From the discussion in Section 2.4.1 it can be concluded that the time-invariant velocity field exists over 50 to 100 subsequent RF returns if the pulse repetition period equals $0.1 \mathrm{~ms}$. In order to have some reserve, $M=25$, or $M=32$ can be considered as an acceptable size of time window. Comparison of the quality measures at $M=25$, or $M=32$ yields the following observation: the behavior of the wideband CCM estimator is stable for all conditions considered, but the behavior of the ML estimator is stable only at lower velocities and for depth windows larger than the transmitted pulse length (Figs. 5.6 (a), (c)).

Figs. 5.7 and 5.8 for the maximum likelihood method and Figs. 6.4 and 6.5 for the wideband cross-correlation model technique show results for nonzero velocity spread. The processing conditions are equivalent to those of Figs. 5.6 and 6.3 . We see that the presence of velocity dispersion has negativer effect on the precision of the ML estimator than has on precision of the wideband CCM method. The bias and standard deviations are so large for the ML estimator that it was necessary to enlarge the zoom window in Figs. 5.7 (b), 5.8 (b), and (d).

Comparing how the estimates approach the imposed value at $M=25$, or $M=$ 
32 , it is evident that the ML estimates exhibit a large bias while the wideband CCM estimates approach the true velocity as can be seen in Figs. 5.7 (a), (b), and 6.4 (a), (b) for a velocity spread of $\vartheta_{\mathrm{s}}=0.1 \lambda / T_{\mathrm{prf}}$. If the velocity spread increases to $\vartheta_{\mathrm{s}}=$ $0.2 \lambda / T_{\text {prf }}$, the ML estimates behave erratically, while the wideband CCM estimator still handles this situation adequately. This estimator is capable of achieving accurate estimates of velocity even when a velocity spread of high value is considered. Compare Figs. 5.8 and 6.5.

Based on the above discussion the following observation concerning precision and temporal resolution can be made: a decrease in the size of time window would improve the temporal resolution but at the same time decrease the estimation precision.

This observation and those from the previous section suggests, that the optimum design of the velocity estimator would encompass systems with a low quality factor, short observation window and short time window. Under these desirable conditions the wideband CCM estimator outperforms the ML estimator significantly.

\subsubsection{Computational Effort}

The methods evaluated in this study can be implemented using correlation processing techniques, though there are substantial differences in the realizations arising from the principles of each method. The maximum likelihood estimator correlates the transformed received signals with a reference signal, whereas the wideband CCM estimator applies correlation to successive returns. In this section the complexity of implementation of each method is approached by evaluating the computational effort in terms of the number of required mathematical operations.

Assuming real-time series sampled in depth at a rate of $R_{\mathrm{S}}$ samples per period then $R_{\mathrm{S}} N_{\mathrm{p}}$ samples become available, where $N_{\mathrm{p}}$ is the size of depth window in periods of carrier frequency. The number of arithmetic operations for a correlation operation at lag $i, C_{i}$, is proportional to the number of samples available and equals

$$
\begin{aligned}
C_{i} & =\left(R_{\mathrm{s}} N_{\mathrm{p}}-i\right)_{\text {multiplications }}+\left(R_{\mathrm{s}} N_{\mathrm{p}}-i-1\right)_{\text {additions }}+1_{\text {division }} \\
& =2 R_{\mathrm{s}} N_{\mathrm{p}}-2 i .
\end{aligned}
$$

Next, the number of operations needed for the maximum likelihood method is evaluated. To start with, the required number of operations for the complex envelope transformation, $\mathcal{O}_{\text {ce }}$, is assessed. It involves $R_{\mathrm{s}} N_{\mathrm{p}}$ multiplications to obtain the imaginary part, and $4 R_{\mathrm{S}} N_{\mathrm{p}}$ multiplications and 3 additions to obtain the complex envelope signal. Thus the total number of operations needed for complex envelope transformation of $M$ signals is

$$
\mathcal{O}_{\text {ce }}=5 R_{\mathrm{s}} N_{\mathrm{p}} M+3 M \text {. }
$$

After signal transformation a correlation procedure is applied for $\nu$ reference signals. In line with our simulation, $\nu=41$, that corresponds to the number of parallel 
branches of the signal processor (Fig. 5.1). Because $M$ complex signals are processed, the number of operations for the maximum likelihood correlation, $\mathcal{O}_{\mathrm{cc}}$ was found to be

$$
\begin{aligned}
\mathcal{O}_{\mathrm{cc}} & =\left(M\left(4 C_{0}+3_{\text {additions }}\right)+2(M-1)_{\text {additions }}+1_{\text {division }}\right) \nu \\
& =\left(8 R_{\mathrm{s}} N_{\mathrm{p}}+M-1\right) \nu .
\end{aligned}
$$

The last processing steps involve the squared magnitude with $3 \nu$ operations, and maximization over $\nu$ values. Then the number of operations at this step, $\mathcal{O}_{\mathbf{v}}$, equals

$$
\mathcal{O}_{\mathrm{v}}=4 \nu
$$

Thus the total number of operations required for the ML estimator is given by summation of (7.4.2), (7.4.3), and (7.4.4), e.g.

$$
\begin{aligned}
\mathcal{O}_{\mathrm{ml}} & =\mathcal{O}_{\mathrm{ce}}+\mathcal{O}_{\mathrm{cc}}+\mathcal{O}_{\mathrm{v}} \\
& =(5+8 \nu) R_{\mathrm{s}} N_{\mathrm{p}} M+(3+\nu) M+3 \nu .
\end{aligned}
$$

The number of operations needed for the wideband CCM method is calculated next. Correlations at six lags (6.3.11) form the basis of the estimator (6.3.22). The number of arithmetical operations required for these correlations using $M$ subsequent signals is given by

$$
\begin{aligned}
\mathcal{O}_{\mathrm{cr}} & =2\left(M C_{0}+(M-1)_{\text {additions }}+1_{\text {division }}\right) \\
& +3\left(M C_{1}+(M-2)_{\text {additions }}+1_{\text {division }}\right) \\
& \left.+M C_{2}++(M-1)_{\text {additions }}+5_{\text {divisions }}\right) \\
& =12 R_{\mathrm{s}} N_{\mathrm{p}} M-4 M+1,
\end{aligned}
$$

where $C_{i}$ for $i=0,1,2$, follows from (7.4.1). The mapping of normalized correlations to the velocity estimate, $(6.3 .22)$ and $(6.3 .17)$, requires 23 mathematical operations to be performed once. It includes summations, additions, divisions, square roots, sign and arctan functions. Hence, the total number of operations required for the wideband $\mathrm{CCM}$ method, $\mathcal{O}_{\mathrm{ccm}}$, is given as the $\mathcal{O}_{\mathrm{cr}}$ value corrected for the number of operations needed for the mapping step. Therefore,

$$
\mathcal{O}_{\mathrm{ccm}}=12 R_{\mathrm{s}} N_{\mathrm{p}} M-4 M+24 \text {. }
$$

The relative size of the expressions (7.4.5), (7.4.7) is clarified by an example with typical settings used in this study. If $\nu=41, M=25, R_{s}=4$, and $N_{\mathrm{p}}=2$ then

$$
\mathcal{O}_{\mathrm{ml}}=67823, \quad \mathcal{O}_{\mathrm{ccm}}=2324 .
$$

So the wideband CCM method is computationally very attractive since it requires approximately $3.4 \%$ of the computations for the ML method. The maximum likelihood estimator is implemented using $\nu$ parallel processing lines, increasing enormously equipment and storage requirements. 
To indicate the processing time needed for one estimate, one parallel branch is considered. Thus $\nu=1$ is substituted into (7.4.5) and the difference between the number of operations of the ML and the wideband CCM methods is given by

$$
\mathcal{O}_{\mathrm{ml}}-\mathcal{O}_{\mathrm{ccm}}=R_{\mathrm{s}} N_{\mathrm{p}} M+8 M-21
$$

which for our representative example has a value of 327 operations. It means that the processing time for the wideband CCM technique is as much shorter as the time required for 327 operations.

We can make the following observations concerning the implementation aspects. Tho wideband CCM method is much simpler to implement than the ML method. It computes correlations at six lags (6.3.10), while correlations at five lags (6.3.3) are required in the narrowband situation. With processing at relatively low sampling rates this does not impose significantly higher computational overheads. Therefore, the wideband CCM estimator is computationally comparable with the narrowband CCM technique. Since the real-time feasibility of the narrowband CCM method has already been reported in Collaris [13], we expect that also the new wideband CCM technique has a great potential to be implemented in real-time.

It suggests that the wideband CCM method can be used in color flow imaging where real-time implementation is required, as well as an acceptable accuracy with good spatial and temporal resolution. 



\section{Chapter 8}

\section{Concluding Remarks}

In this chapter we give an outline of the main contributions of the thesis and of the possible directions for future research.

The thesis presents a study of blood velocity estimation using pulsed ultrasound echo signals backscattered from blood. The goal of this study was to investigate the performance of the existing methods for processing of backscattered signals for blood velocity estimation, and to compare various techniques in an identical way for the effect of processing and system parameters such as the signal-to-noise ratio and the quality factor of the transducer. Furthermore, the goal was to propose new processing techniques for possible improvement in accuracy and spatial resolution of ultrasound blood velocity measurements. These aims have been achieved by encompassing the evaluation and comparison of two processing methods, the maximum likelihood (ML) method and the cross-correlation model (CCM) method and by extending the crosscorrelation model method to a wideband situation yielding a new wideband CCM estimator. The resulting gain in accuracy of the new technique for use with wideband signals is desirable for simultaneous improvement of accuracy and axial resolution.

In this thesis we have started (Chapter 2) with a survey of the physical principles relevant for ultrasound blood flow velocity measurements. Particular attention has been given to the features of signals backscattered from blood. Backscattered signals, received in pulsed wave measurement mode, form a set of radio frequency $(\mathrm{RF})$ signals that are separated by the pulse repetition period. Due to the changing distance between the transducer and groups of moving scatterers (red blood cells), each subsequent signal exhibits gradual translation with respect to the previous signal. This translation carries information about the velocity of moving scatterers. By suitable processing of the sequence of RF signals, the required information can be extracted. A number of techniques can be employed to extract velocity information from backscattered signals. The most salient have been summarized in Chapter 3 for the case of processing low and high frequency signals, according to a proposed classification scheme based on the type of input data. The simulation procedure for computer generation of signals having the above mentioned properties has been 
presented in Chapter 4 . The simulation model of the backscattered signal from blood has provided data for the analysis of various methods in the subsequent chapters.

Two alternative methods have been evaluated. The maximum likelihood method has been re-examined in Chapter 5. The cross-correlation model method for narrowband signals has been extended to a new wideband situation in Chapter 6 . Comparison of the performance of both narrowband and wideband CCM estimators has shown that for narrowband signals both CCM estimators behave in similar way, while for wideband signals, the new wideband CCM estimator is superior.

In Chapter 7 two alternative methods (maximum likelihood and wideband CCM) have been compared directly in statistical terms (bias and standard deviation) obtained from the Monte Carlo simulations. The results have been compared at identical situations, determined primarily by the signal-to-noise ratio, quality factor, mean velocity, velocity dispersion, depth window length, and time window size.

To investigate the relevance of the estimation methods, we had to resort to computer simulations based on the Monte Carlo method. The simulations were implemented on a $\mathrm{PC}$, using software package Matlab ${ }^{1}$.

The backscattered signals generated by a procedure described in Chapter 4 have been realizations of a random process. We have constructed a large number of independent realizations and to each one the same procedure has been applied. Application of the procedure defined by the studied estimator (ML or CCM) has yielded a large number of velocity estimates, from which the statistical properties of the studied estimator have been obtained. It was possible to investigate the effects of numerous different processing parameters since the underlying averaged imposed properties (mean velocity, velocity dispersion, signal-to-noise ratio, quality factor) of the simulated process were completely known. A qualitative assessment of the performance of the reported estimators has been achieved by a comparison of the resulting statistical measures to the imposed values. In practice, it is very difficult to evaluate real data properly, given the unknown and time-varying nature of both the volume flow and velocity profiles.

This study allows for a global comparison of the reported methods so that the differences and similarities between two estimators can be revealed. Conceptually, the reported estimators involve different ideas (maximum likelihood criterion and the cross correlation concept) and various assumptions. The signal processors based on these estimators employ correlation of the data, though in a different way. The maximum likelihood method derives a velocity estimate from correlating the preprocessed version of the observed $\mathrm{RF}$ signal with a reference (model) signal, whereas the wideband CCM processor correlates a number of successive RF signals.

The ML and the wideband CCM techniques represent two classes of processing methods, distinguished by the type of input data they operate on. ML operates on low frequency, complex signals obtained by pre-processing the observed signals by

\footnotetext{
${ }^{1}$ Matlab is a registrated trademark of the Math Works Inc.
} 
phase detection. In contrast, the wideband CCM method maintains the real nature of the high frequency signals. The fact that the CCM approach does not involve signal transformation circumvents the problem of assuming a reference frequency.

The detailed investigation of each of the considered methods yields several conclusions with respect to their performance and applicability. The evaluation of the maximum likelihood method in Section 5.4 indicates an acceptable performance only for a limited range of signal and processing conditions (high signal-to-noise ratio, large sizes of spatial and temporal windows, absence of velocity spread, low mean velocities). This poor performance is attributed to the fundamental assumption of this method - "the slowly fluctuating point target approximation". This assumption causes incompatibility between the assumed model of the backscattered signal (the refere ice signal) and the signal actually observed. An improvement can be achieved by specific post-processing, like median filtering, which however degrades the temporal resolution.

The performance of the wideband CCM method is evaluated in Section 6.5 indicating good results over a fairly wide range of signal and processing parameters. The method is shown to be robust to some extent for the assumption about a symmetric shape of the spectrum (Section 6.5.4).

A comparative evaluation of the ML and the wideband CCM techniques under the same conditions and in an identical way is accomplished. Overall, the wideband CCM exhibits superior performance. The wideband CCM estimator is found to offer a consistently higher velocity precision than the ML estimator under all considered conditions (Section 7.4 ). The results of Section 7.2 show that the maximum likelihood estimator suffers from the effect of frequency-dependent attenuation due to the fact which causes a significant bias in the estimate. In contrast, the wideband cross-correlation model method estimator is not influenced by frequency-dependent effects. Furthermore, the efficiency of the wideband CCM estimator is demonstrated in Section 7.3 by the fact that the standard deviation resulting from this estimator approaches the theoretical limit given by the Cramér-Rao lower bound very closely.

The results of this study suggest that the conflicting requirements on precision and resolution (axial and temporal) can be satisfactorily met to some extent using the new wideband CCM method. Implementation can encompass systems with low quality factor, short observation windows and short time windows which is desirable for an improved spatial and temporal resolution. It was demonstrated in Section. 7.4.3 that the real-time implementation seems to be feasible for the wideband CCM estimator. Therefore, this new technique has a great potential for application in color flow imaging technology.

There are several possible directions in which this research could be continued, of which we mention the following.

First, this comparative study can be extended by including for other methods for blood velocity assessment. The comparison of methods under the same conditions and in the identical way would help to identify the best technique at a given condition. 
[11] P. J. Brands, Non-invasive methods for the assessment of wall shear rate and arterial impedance. PhD thesis, University of Limburg, Department of Biophysics, PO Box 616, 6200 MD Maastricht, The Netherlands, May 1996.

[12] I. Céspedes, Y. Huang, J. Ophir, and S. Spratt, "Methods for estimation of subsample time delays of digitized echo signals," Ultrasonic Imaging, vol. 17, pp. 142-171, 1995.

[13] R. J. Collaris, New Concepts in Ultrasound Image processing and their potential for improving data presentation. $\mathrm{PhD}$ thesis, University of Limburg, Department of Biophysics, PO Box 616, 6200 MD Maastricht, The Netherlands, June 1996.

[14] P. G. M. de Jong, Measurement of Myocardial Tissue Deformation by Correlation Interpolation of Pulsed Ultrasonic Echo Signals. PhD thesis, University of Limburg, Department of Biophysics, PO Box 616, 6200 MD Maastricht, The Netherlands, May 1991.

[15] P. G. M. de Jong, T. Arts, A. Hoeks, and R. S. Reneman, "Determination of tissue motion velocity by correlation interpolation of pulsed ultrasonic echo signals," Ultrasonic Imaging, vol. 12, pp. 84-98, 1990.

[16] P. M. Embree and W. D. O'Brien, "Pulsed Doppler accuracy assessment due to frequency dependent attenuation and Raleigh scattering error sources," IEEE Trans. Biomed. Eng., vol. 37, pp. 322-326, 1990.

[17] D. H. Evans, W. N. McDicken, R. Skidmore, and J. P. Woodcock, Doppler Ultrasound: Physics, Instrumentation, and Clinical Applications. Chichester: John Wiley \& Sons, 1989.

[18] R. L. Fante, Signal Analysis and Estimation: An Introduction. John Wiley \& Sons, Inc, 1988.

[19] K. W. Ferrara and V. R. Algazi, "A new wideband spread target maximum likelihood estimator for blood velocity estimation-Part one: Theory," IEEE Trans. Ultrason., Ferroelec. Freq. Contr., vol. 38, pp. 1-16, 1991.

[20] K. W. Ferrara, V. R. Algazi, and J. Liu, "The effect of frequency dependent scattering and attenuation on the estimation of blood velocity using ultrasound," IEEE Trans. Ultrason., Ferroelec. Freq. Contr., vol. 39, pp. 754-767, 1992.

[21] K. W. Ferrara and W. R. Algazi, "A new wideband spread target maximum likelihood estimator for blood velocity estimation-Part two: Evaluation of estimators with experimental data," IEEE Trans. Ultrason., Ferroelec. Freq. Contr., vol. 38, pp. 17-26, 1991. 
[22] K. W. Ferrara, B. G. Zagar, J. B. Sokil-Melgar, R. H. Silverman, and I. A. Aslanidis, "Estimation od blood velocity with high frequency ultrasound," IEEE Trans. Ultrason., Ferroelec. Freq. Contr., vol. 43, pp. 149-156, 1996.

[23] S. G. Foster, P. M. Embree, and W. D. O'Brien, "Flow velocity profile via time-domain correlation: error analysis and computer simulation," IEEE Trans. Ultrason., Ferroelec. Freq. Contr., vol. 37, pp. 164-175, 1990.

[24] J. H. Halton, "A retrospective and prospective survey of the Monte Carlo method," SIAM Review, vol. 12, pp. 1-63, 1970.

[25] L. Hatle and B. Angelsen, Doppler Ultrasound in Cardiology. Philadelphia: Lea \& Febiger, 1982.

[26] I. A. Hein, J. T. Chen, W. K. Jenkins, and W. D. O'Brien, "A real-time ultra" sound time-domain correlation blood flowmeter: Part I - theory and design," IEEE Trans. Ultrason. Ferroelectr. Freq. Contr., vol. 40, pp. 768-775, 1993.

[27] I. A. Hein. J. T. Chen, W. K. Jenkins, and W. D. O'Brien, "A real-time ultrasound time-domain correlation blood flowmeter: Part II - performance and experimental verification," IEEE Trans. Ultrason. Ferroelectr. Freq. Contr., vol. 40, pp. 776-785, 1993.

[28] I. A. Hein and W. D. O'Brien, "Current time-domain methods for assessing tissue motion by analysis from reflected ultrasound echoes - a review," IEEE Trans. Ultrason. Ferroelectr. Freq. Contr., vol. 40, pp. 84-102, 1993.

[29] A. P. G. Hoeks, T. G. J. Arts, P. J. Brands, and R. S. Reneman, "Comparison of the performance of the RF cross correlation and Doppler autocorrelation technique to estimate the mean velocity of simulated ultrasound signals," Utirasound in Med. \& Biol., vol. 19, pp. 727-740, 1993.

[30] A. P. G. Hoeks, P. J. Brands, T. G. J. Arts, and R. S. Reneman, "Subsample volume processing of Doppler ultrasound signals," Ultrasound in Med. E' Biol., vol. 20, pp. 953-965, 1994.

[31] A. P. G. Hoeks, P. J. Brands, and R. S. Reneman, "Effect of sample window length on the correlation between rf signal and pulsed Doppler signal intensity," Ultrasound in Med. ES Biol., vol. 20, pp. 35-40, 1994.

[32] A. P. G. Hoeks, M. Hennerici, and R. S. Reneman, "Spectral composition of Doppler signals," Ultrasound in Med. \& Biol., vol. 17, pp. 751-760, 1991.

[33] D. Hykes, W. R. Hedrick, and D. E. Starchman, Ultrasound Physics and Instrumentation. Churchill Livingstone, 1985. 
[34] J. A. Jensen, "Implementation of ultrasound time-domain cross-correlation blood velocity estimators," IEEE Trans. Biomed. Eng., vol. 40, pp. 468-473, 1993.

[35] J. A. Jensen, "Range/velocity limitation for time-domain blood velocity estimation," Ultrasound in Med. \& Biol., vol. 19, pp. 741-749, 1993.

[36] S. A. Jones, "Fundlamental sources of error and spectral broadening in Doppler ultrasound signals," Critical Reviews in Biomed. Engin., vol. 21, pp. 399-483, 1993.

[37] S. A. Jones and P. D. Giddens, "A simulation of transit time effects in Doppler ultrasound signals," Ultrasound in Med. \& Biol., vol. 16, pp. 607-619, 1990.

[38] C. Kasai, K. Namekawa, A. Koyano, and R. Omoto, "Real-time two dimensional blood flow imaging using an autocorrelation technique," IEEE Trans. Sonics Ultrason., vol. 32, pp. 458-464, 1985.

[39] C. H. Knapp and G. C. Carter, "The generalized correlation method for estimation of time delay," IEEE Trans. Acoust. Speech, Signal Processing, vol. 24, pp. 644-654, 1976.

[40] K. Kristoffersen, "Optimal receiver filtering in pulsed Doppler ultrasound blood velocity measurements," IEEE Trans. Ultrason., Ferroelec. Freq. Contr., vol. 33, pp. 51-58, 1986.

[41] K. Kristoffersen, "Time-Domain estimation of the center frequency and spread of Doppler spectra in diagnostic ultrasound," IEEE Trans. Ultrason., Ferroelec. Freq. Contr., vol. 35, pp. 484-497, 1988.

[42] H. Kwakernaak and R. Sivan, Modern Signals and Systems. Prentice-Hall International, Inc., 1991.

[43] L. Ljung, System Identification: Theory for the User. Prentice-Hall, Inc., 1987.

[44] T. Loupas and R. W. Gill, "Multifrequency Doppler: improving the quality of spectral estimation by making full use of the information present in the backscattered RF echoes," IEEE Trans. Ultrason., Ferroelec. Freq. Contr., vol. 41, pp. $522-531,1994$.

[45] T. Loupas, R. B. Peterson, and R. W. Gill, "Experimental evaluation of velocity and power estimation for ultrasound blood flow imaging, by means of a twodimensional autocorrelation approach," IEEE Trans. Ultrason., Ferroelec. Freq. Contr., vol. 42, pp. 689-699, 1995.

[46] T. Loupas, J. Powers, and R. W. Gill, "An axial velocity estimator for ultrasound blood flow imaging, based on a full evaluation of the Doppler equation by means of a two- dimensional autocorrelation approach," IEEE Trans. Ultrason., Ferroelec. Freq. Contr., vol. 42, pp. 672-688, 1995. 
[47] R. Luesink, The Likelihood Ratio for Two-Parameter Stochastic Processes. PhD thesis. University of Twente, Department of Applied Mathematics, PO Box 217, 7500 AE Enschede, The Netherlands, March 1992.

[18] L. Y. L. Mo and R. S. C. Cobbold, "A stochastic model of the backscattered Doppler ultrasound from blood," IEEE Trans. Biomed. Eng., vol. 33, pp. 20-27, 1986.

[49] L. Y. L. Mo and R. S. C. Cobbold, "A unified approach to modeling the backscattered Doppler ultrasound from blood," IEEE Trans. Biomed. Eng., vol. 39, pp. 450-461, 1992.

[50] N. Mohanty, Random Signals Estimation and Identification: Analysis and Applications. Van Nostrand Reinhold Company Inc., 1986.

[51] V. L. Newhouse, W. Varner, and P. J. Bendick, "Greometrical spectrum broadening in ultrasonic Doppler systems," IEEE Trans. Biomed. Eng., vol. 24, pp. 478$480,1977$.

[52] P. Z. Peebles, Probability, Random Variables, and Random Signal Principles. Singapore: McGraw-Hill, 1987.

[53] M. B. Priestley, Spectral Analysis and Time Series. London: Academic Press, 1981.

[54] A. H. Quazi, "An overview on the time delay estimate in active and passive systems for target localization," IEEE Trans. Acoust. Speech, Signal Processing, vol. 29, pp. 527-533, 1981.

[55] C. R. Rao, Linear Statistical Inference and Its Applications. John Wiley \& Sons, Inc, 1973.

[56] K. K. Shung, "General engineering principles in diagnostic ultrasound," IEEE Engng in Med. \& Biol. Mag., vol. 6, pp. 7-13, 1987.

[57] T. Söderström and P. Stoica, System Identification. Prentice-Hall International (UK) Ltd, 1989.

[58] H. E. Talhami and R. I. Kitney, "Maximum likelihood frequency tracking of the audio pulsed Doppler ultrasound signal using a Kalman filter," Utitrasound in Med. Biol., vol. 14, pp. 599-609, 1988.

[59] H. Torp, K. Kristoffersen, and B. A. J. Angelsen, "Autocorrelation techniques in ultrasound color flow imaging. signal model and statistical properties of the autocorrelation estimates," IEEE Trans. Vltrason., Ferroelec. Freq. Contr., vol. 41, pp. 604-612, 1994. 
[60] H. Torp, K. Kristoffersen, and B. A. J. Angelsen, "On the joint probability density function for the autocorrelation estimates in ultrasound color flow imaging," IEEE Trans. Ultrason., Ferroelec. Freq. Contr,, vol. 42, pp. 899-906, 1995.

[61] P. J. Vaitkus and R. S. C. Cobbold, "A comarative study of assessment of Doppler ultrasound spectral estimation techniques. Part I: Estimation methods," Ultrasound in Med. \& Biol., vol. 14, pp. 661-672, 1988.

[62] H. L. van Trees, Detection, Estimation and Modulation Theory, Part I. New York: Wiley, 1971.

[63] H. L. van Trees, Detection, Estimation and Modulation Theory, Part III. New York: Wiley, 1971.

[64] E. Śtúriková, A. P. G. Hoeks, and R. Luesink, "Blood velocity estimation by maximum likelihood: a comparative study," in Proc. 17th Annu. Int. Conf. $I E E E / E M B C$, pp. 945-946, 1995.

[65] E. Śtúriková, A. P. G. Hoeks, and R. Luesink, "Maximum likelihood estimation of blood velocity in perspective," Report M 95-12, University of Limburg, Department of Mathematics, PO Box 616,6200 MD Maastricht, The Netherlands, December 1995.

[66] E. Stúriková, A. P. G. Hoeks, and R. Luesink, "Wideband cross correlation model method for blood velocity estimation," in Proc. 18th Annu. Int. Conf. IEEE/EMBC, pp. 912-913, 1996.

[67] W. F. Walker and G. E. Trahey, "A fundamental limit on the performance of correlation based phase correction and flow estimation techniques," IEEE Trans. Ultrason., Ferroelec. Freq. Contr, vol. 41, pp. 644-654, 1994.

[68] W. F. Walker and G. E. Trahey, "A fundamental limit on delay estimation using partially correlated speckle signals," IEEE Trans. Ultrason., Ferroelec. Freq. Contr., vol. 42, pp. 301-308, 1995.

[69] P. N. T. Wells, Biomedical Ultrasonics. London: Academic Press, 1977.

[70] P. N. T. Wells, "History and development," in Cardiac Ultrasound, (J. R. T. C. Roelandt, G. R. Sutherland, S. Ilicet,o, and D. T. Linker, eds.), pp. 3-8, Churchill Livingstone, 1993.

[71] L. S. Wilson, "Description of broad-band pulsed Doppler ultrasound processing using the two-dimensional Fourier transform," Ultrasonic Imaging, vol. 13, pp. 301-315, 1991. 


\section{Summary}

\section{Correlation Based Methods for Ultrasound Blood Velocity Estimation}

This thesis presents a study of blood velocity estimation using pulsed ultrasound echo signals backscattered from blood. Blood velocity is an important parameter in diagnosing vascular diseases since changes in blood flow caused by pathology (i.e. atherosclerosis) can be directly observed through changes in the speed of blood.

In pulsed wave measurement mode the transducer emits ultrasound pulses with pulse repetition period. Between transmissions, the transducer is switched to reception mode to catch the signals backscattered from blood. The received backscattered signals are aligned to form a set of radio frequency ( $\mathrm{RF}$ ) signals that are separated by the pulse repetition period. Due to the changing distance between the transducer and groups of moving scatterers (red blood cells), each subsequent signal exhibits gradual translation with respect to the previous signal. This translation carries information about the velocity of moving scatterers. By suitable processing of a sequence of observed RF signals, the required information can be extracted. A number of techniques can be employed to extract velocity information from such signals. However, the diversity of descriptions and assumptions of the results reported in the literature do not allow a straight comparison of their performance. Despite this, it can be recognized that the drawbacks of the existing processing methods include limitations arising from the conflicting requirements for velocity precision, and resolution in the spatial and temporal domain.

The goal of this study was to investigate the performance of the existing methods for processing of backscattered signals for blood velocity estimation, and to compare various techniques in an identical way for the effect of processing and sys. tem parameters such as the signal-to-noise ratio and the quality factor of the transducer. Furthermore, the goal was to propose new processing techniques for possible improvement in accuracy and spatial resolution of ultrasound blood velocity measurements. These aims have been achieved by encompassing the evaluation and comparison of two processing methods, the maximum likelihood (ML) method and the cross-correlation model (CCM) method and by extending the cross-correlation model method to a wideband situation yielding a new wideband CCM estimator. The resulting gain in accuracy of the new technique for use with wideband signals is desirable for simultaneous improvement of accuracy and axial resolution. 
This study allows for a comparison of the reported methods. Conceptually, the reported estimators involve different ideas (maximum likelihood criterion and the cross correlation concept) and various assumptions. The signal processors based on these estimators employ correlation of the data, though in a different way. The maximum likelihood method derives a velocity estimate from correlating the pre-processed version of the observed RF signal with a reference (model) signal, whereas the wideband CCM processor correlates a number of successive RF signals. The ML and the wideband CCM techniques represent two classes of processing methods, distinguished by the type of input data they operate on. ML operates on low frequency, complex signals obtained by pre-processing the observed signals by phase detection. In contrast, the wideband CCM method maintains the real nature of the high frequency signals. The fact that the CCM approach does not involve signal transformation circumvents the problem of assuming a reference frequency.

A simulation study was designed in order to evaluate the effects of the system and processing parameters on the accuracy of the velocity estimators. The evaluation of the maximum likelihood method indicates an acceptable performance only for a limited range of signal and processing conditions. This poor performance is attributed to the fundamental assumption of this method - "the slowly fluctuating point target approximation". This assumption causes incompatibility between the assumed model of the backscattered signal (the reference signal) and the signal actually observed. The performance of the new wideband CCM method is also evaluated, indicating good results over a fairly wide range of signal and processing parameters. A comparative evaluation of the ML and the wideband CCM techniques under the same conditions and in an identical way is accomplished. The wideband CCM estimator is found to offer a consistently higher velocity precision than the ML estimator under all conditions considered. Furthermore, the efficiency of the wideband CCM estimator is demonstrated by the fact that the standard deviation resulting from this estimator approaches the theoretical limit given by the Cramér-Rao lower bound closely.

The results of this study suggest that the conflicting requirements on precision and resolution (axial and temporal) can be satisfactorily met to some extent using the new wideband CCM method. Since the real-time implementation seems to be feasible for the processing procedure, this new technique has a great potential for application in color flow imaging technology.

Possible directions for future research are threefold. First, this comparative study might be continued by including other methods for blood velocity estimation. The second possibility would be the experimental evaluation of the methods with real data. The third direction of interest would be to study the possibilities of the wideband cross-correlation model method in other fields with similar types of problems. A typical task would be to assess the mean velocity of a moving substance by processing signals originating from a large number of moving elements of that substance. 


\section{Samenvatting}

\section{Correlatie Gebaseerde Methoden voor Bloedsnelheidschatting met Ultrageluid}

Dit proefschrift beschrijft een onderzoek naar schattingsmethoden voor bloedsnelheden. Hierbij wordt gebruik gemaakt van echosignalen, die ontstaan doordat uitgezonden ultrageluidpulsen worden verstrooid (backscattered) door de rode bloedcellen. Bloedsnelheid is een belangrijke parameter voor de diagnose van vaatziekten, omdat veranderingen in het stromingspatroon, veroorzaakt door pathologie (bijv. atherosclerosis) direct waarneembaar is door veranderingen in de bloedsnelheid.

Echosignalen, veroorzaakt door verstrooiing, worden verkregen door uitzending van pulsen. Ze vormen een reeks van radio-frequentie (RF) signalen, onderling gescheiden door de tijdsduur tussen de pulsen, de pulse repetitie periode. Door de veranderende afstand tussen de transducer (de zender en ontvanger van de signalen) en de bewegende verstrooiiers (de rode bloedcellen), toont ieder opeenvolgend ontvangen echosignaal een graduele translatie ten opzichte van het voorgaande echosignaal. Deze translatie bevat informatie over de snelheid waarmee de bloedcellen zich voortbewegen. Door een adequate bewerking op de reeks van echosignalen kan deze snelheid worden bepaald. Er bestaat een aantal technieken om deze bloedsnelheid te bepalen. De diversiteit in de beschrijving en gestelde voorwaarden voor deze methoden, zoals ze in de literatuur beschreven zijn, maken een directe vergelijking van de methoden echter onmogelijk. Desondanks kan worden vastgesteld dat de belangrijkste beperkingen van de bestaande schattingsmethoden worden gevormd door de tegenstrijdige eisen van precisie van de schatters enerzijds, en resolutie in ruimtelijk en tijd domein anderzijds.

Het doel van het onderzoek beschreven in dit proefschrift is een antal van de bestaande methoden voor bloedschatting te vergelijken, uiteraard onder gelijke omstandigheden, en hun werkzaamheid te beschouwen ten aamzien van verschillende systeemparameters zoals de signaal-ruis-verhouding en de kwaliteitsfactor van de transducer. Een tweede doel van het onderzoek is om nieuwe technieken te ontwikkelen met mogelijk verbeterde resolutie in ruimte en tijd. Deze doelen zijn behaald, in de zin dat dit proefschrift een conceptuele basis, evaluatie en vergelijking van twee schattingsmethoden bevat, de Maximum Likelihood (ML) en de Cross-Correlation Model (CCM) methode. Bovendien is de Cross-Correlation Model methode aangepast naar een breedband situatie. Deze nieuwe ontwikkeling leidt tot een gelijktijdige verbetering van de precisie van de schatter, alsmede de axiale resolutie. 
Het onderzoek maakt het mogelijk om de beschreven methoden te vergelijken. Conceptueel bevatten de methoden verschillende ideeën (maximale waarschijnlijkheid en kruiscorrelatie), maar beide methoden gebruiken de correlatie van signalen. $\mathrm{Bij}$ de Maximum Likelihood methode vindt correlatie plaats tussen een voorbe werkte versie van het echosignaal en een referentie (model) signaal. Bij de Cross-Correlation Model methode vindt correlatie plaats tussen opeenvolgende echosignalen. De Maximum Likelihood methode maakt gebruik van laagfrequente complexe signalen, die worden verkregen door voorbewerking van het echosignaal, terwijl de nieuwe breedband Cross-Correlation Model methode gebruik maakt van het oorspronkelijke hoogfrequente echosignaal. Dit laatste voorkomt de noodzaak tot een veronderstelling ten aanzien van de referentiefrequentie van het echosignaal.

Om het effect te bestuderen van verschillende parameters op de kwaliteit van de snelheidschatting, zijn uitgebreide simulaties uitgevoerd. De evaluatie voor de Maximum Likelihood methode geeft aan dat de kwaliteit van de schatter acceptabel is voor een beperkt bereik van de parameters. Deze zwakke prestaties worden toegemeten aan een fundamentele veronderstelling bij de Maximum Likelihood methode - de "slowly fluctuating point target approximation". Deze veronderstelling veroorzaakt een afwijking tussen het model voor het echosignaal (het referentie signaal) en het werkelijk waargenomen signaal. Ook het gedrag van de breedband Cross-Correlation Model methode is onderzocht voor verschillende parameterwaarden, hetgeen heeft geleid tot goede schattingsresultaten over een breed parameterbereik. Een vergelijkend onderzoek tussen de Maximum Likelihood methode en de breedband Cross-Correlation Model methode leidt tot de conclusie dat de breedband CCM methode een consistent hogere precisie levert onder alle beschouwde parameter condities. Bovendien blijkt dat de breedband Cross-Correlation Model methode een schatter oplevert die efficient is in de zin dat de kwaliteit, uitgedrukt in de statistische variantie, dicht bij het theoretisch minimum, de Cramer Rao ondergrens zit.

De resultaten van het onderzoek geven aan dat de tegenstrijdige voorwaarden tussen precisie van de schatter en resolutie (tijd en axiaal) tot tevredenheid kunnen worden verenigd door middel van de breedband Cross-Correlation Model methode. Daar bovendien real-time implementatie haalbaar lijkt te zijn voor de schattingsprocedure, heeft deze techniek een groot potentieel voor toepassing in de "color flow imaging" technologie.

Drie mogelijke richtingen voor toekomstig onderzoek zijn de volgende. Ten eerste, het vergelijkend onderzoek kan worden uitgebreid naar andere schattingsmethoden. Een tweede richting voor verder onderzoek is een evaluatie van de methoden met behulp van reële meetdata, alsmede een practische implementatie van de breedband Cross-Correlation Model methode. Tenslotte kan worden onderzocht hoe de breedband Cross-Correlation Model methode toepasbaar kan worden gemaakt naar andere schattingsproblemen. Typisch voor geschikte toepassingen is, dat de gemiddelde snelheid dient te worden geschat van een bewegende substantie, waarbij het meetsignaal wordt opgebouwd uit signalen afkomstig van een groot aantal bewegende elementen binnen de substantie. 


\section{Zhrnutie}

\section{Korelačné metódy na ultrazvukové odhadovanie rýchlosti krvi}

Predmetom štúdia tejto dizertácie je odhadovanie rýchlosti toku krvi na základe ultrazvukových signálov spätne rozptýlených (odrazených) krvou. Rýchlost krvi je dôležitý diagnostický parameter pre kardiovaskulárne choroby, pretože zmeny v prúdení krvi zapríčinené patologickými procesmi (napríklad atherosclerosis) sú priamo pozorovateliné cez zmeny $v$ rýchlosti toku krvi.

Po vyslaní ultrazvukového impulzu získame signál naspät' odrazený krvou. Vyslaný impulz sa rozptýli od pohybujúcich sa červených krviniek a je spätne prijatý prijimačom. Elektromechanický menič (vysielač aj prijímač) vysiela obyćajne periodicky viac impulzov za sebou. S' tou istou periódou sú potom prijímané aj echo signály. Prijaté signály sú zosynchronizované s periódou opakovania impulzov a vytvárajú tak postupnosṫ rádio-frekvenčných ( $R F$ ) signálov. Pretože vzdialenost' medzi meničom a skupinami pohybujúcich sa červených krviniek sa mení, každý prijatý signál je posunutý od predchádzajúceho. Toto posunutie je fyzikálnym nositel'om informácie o priemernej rýchlosti, s ktorou sa krvinky pohybujú. Požadovanú informáciu je možné získat vhodným spracovaním postupnosti RF signálov. Existujú rôzne spôsoby ako stanovit' rýchlost' toku krvi, avšak rozdielnost' v popise a v predpokladaných podmienkach nedovoluje ich priame porovnanie. Jedno z hlavných obmedzení existujúcich metód však spočíva v konfliktných požiadavkách na presnost' a rozlíšitelinost' (priestorovú a časovú).

Cielom tohto výskumu bolo preskúmat niektoré existujúce metódy na spracovanie signálov za úćclom zistenia rýchlosti toku krvi a porovnat tieto metódy za rovnakých podmienok v závislosti od viacerých parametrov, ako napríklad od pomeru signál-šum, a od šírky vlnového pásma meniča. Dalším cielom tohto výskumu bolo navrhnút nový postup, ktorého výsledkom by bolo súčasné zlepšenie presnosti odhadu a rozlísitel'nosti $v$ priestore a čase. Tieto ciele boli dosiahnuté vyhodnotením a porovnanim dvoch metód, metódy maximálnej vierohodnosti (ML) a metódy modelovanej vzájomnej korelácie (CCM) a rozširením metódy modelovanej vzájomnej korelácie na širokopásmové signály, čím sa obdržal nový spôsob stanovenia priemernej rýchlosti toku krvi. Tento nový odhad vedie k zvýšeniu presnosti pri použití širokopásmových signálov, čo má za následok zlepšenie rozlíšitelnosti v priestore pri zobrazovaní nameraných údajov. 
Na základe výsledkov nášho výskumu je možné porovnat (vy̌̌šie) uvedené metódy. Koncepčne sú tieto metódy založené na rôznych princípoch (maximálna vierohodnost' a vzájomná korelácia) a vyžadujú odlišné predpoklady. Spracovanie signálov vs̉ak vedie pre obidve metódy $\mathrm{k}$ uplatneniu korelácie, i ked' rozličným spôsobom. Pre metódu maximálnej vierohodnosti je charakteristické, že výsledný odhad je získaný koreláciou transformovaného echo signálu s referenčným, zatial co metóda modelovanej vzájomnej korelácie koreluje prijaté echo signály medzi sebou. Uvedené spôsoby spracovania signálov predstavujú dve kategórie metód rozlíšitelných. podla typu signảlov, ktoré spracúvajú. Metóda maximálnej vierohodnosti používa nízkofrekvenčné, komplexné signály získané demoduláciou prijatého signálu vzhladom na referenčnú frekvenciu. Na druhej strane, metóda modelovanej vzájomnej korelácie spracúva pôvodné vysokofrekvenčné signály a teda znalost referenčnej frekvencie nie je nutná.

Za účelom štatistického vyhodnotenia efektu rozdielnych parametrov na kvalitu odhadu rýchlosti sme uskutočnili viaceré simulácie. Výsledky dosiahnuté metódou maximálnej vierohodnosti naznačujú obmedzené použitie tohto postupu, len pre niektoré hodnoty uvažovaných parametrov. Za hlavnú príčinu je považovaný základný predpoklad tejto metódy, č je - "aproximácia pomaly sa pohybujúcim bodom". Táto aproximácia spôsobuje nekompatibilitu medzi modelom echo signálu (referenčným signálom) a skutočným pozorovaným signálom. Vyhodnotenie širokopásmovej verzie metódy modelovanej vzájomnej korelácie naznačuje jej použitelnost' pre širokú škálu hodnôt parametrov. Porovnaním výsledkov týchto dvoch metód sme dospeli k záveru, že nová širokopásmová metóda modelovanej vzájomnej korelácie poskytuje vyššiu presnost odhadov než metóda maximálnej vierohodnosti a je použitel’ná pre široký rozsah parametrov. Navyše, účinnost novej metódy bola demonštrovaná tým, že štatistická odchýlka odhadu získaného touto metódou takmer dosahuje teoretickú minimálnu hodnotu danú Cramér-Raovým dolným ohraničením.

Výsledky našej štúdie ukazujú, že protikladné požiadavky na presnost odhadu a priestorovú rozlísitelnost' je možné do určitej miery splnit použitím novej širokopásmovej metódy modelovanej vzájomnej korelácie. A kedže implementácia tejto metódy sa zdá byt uskutočnitelná v reálnom čase, má táto metóda spracovania signálov dobré predpoklady na aplikáciu v technológii "farebné zobrazovanie toku".

Možnosti pre budúci výskum vidime trojaké. Prvá možnost by bola pokračovat $\checkmark$ tejto štúdii zahrnutím iných metód na odhadovanie rýchlosti toku krvi. Drubý smer budúceho výskumu by bolo vyhodnotenie metód použitím skutočných nameraných signálov a praktická implementácia novej širokopásmovej metódy modelovanej vzájomnej koreläcie. Tretím smerom záujmu by mohol byt prieskum možností aplikácie novej metódy v iných oblastiach na podobný typ problémov. Typickou úlohou by bolo určenie priemernej hodnoty rýchlosti pohybujúcej sa hmotnej substancie na základe signálov odrazených z velkého počtu pohybujúcich sa častíc tejto substancie. 


\section{About the Author}

Elena Štúriková was born on November 4th, 1965 in Trenčianske Teplice, Slovakia. She finished her secondary education in 1984 by succeeding in the leaving examination at the Gymnázium Dubnica nad Váhom.

In 1989 she graduated with honors from the Faculty of Electrical Engineering and Information Technology of the Slovak Technical University in Bratislava. She acquired a university qualification equivalent to a Master's degree in the field of automated systems of control. After graduation she was appointed as assistant university teacher at the Department of Automated Systems of Control at the same university.

She was selected to undertake a study programme in Electronic Engineering at the Eindhoven International Institute, successor to the Philips International Institute. She started these studies in January 1991, at that time the institute being a part of the Eindhoven University of Technology in The Netherlands. After successful completion of the theoretical and practical parts of the study programme she acquired the Master's Degree in May 1992. This degree was awarded by the Netherlands University Foundation for International Cooperation in Higher Education (NUFFIC).

Since September 1992 she has been a member of the Department of Mathematics of the Faculty of General Science, Maastricht University, The Netherlands. Her position is that of a research assistant. A synthesis of her research work is presented in this academic dissertation. 
ISBN 90-9010267-1 